\title{
Cellular Defects in Hutchinson-Gilford Progeria Syndrome
}

\author{
Sutirtha Datta \\ Kolkata, India
}

BS Microbiology, University of Pune, India, 2005

MS Life Sciences, Jawaharlal Nehru University, India, 2007

A Dissertation presented to the Graduate Faculty of the University of Virginia in Candidacy for the Degree of Doctor of Philosophy

Department of Biochemistry and Molecular Genetics

University of Virginia

August 2013 


\section{ABSTRACT}

The Hutchinson-Gilford Progeria Syndrome(HGPS) is a premature aging syndrome in children that is caused by the disruption of the nuclear lamina owing to the expression of Progerin, a mutant form of the Lamin A protein. This leads to altered physical properties of the lamina causing changes in nuclear morphology. Additionally there are structural alterations in chromatin along with global changes in gene expression patterns. However the intermediate mechanisms linking lamina perturbation to the above phenotypes have been a subject of investigation. The Ran protein gradient and nuclear SUMOylation are two fundamentally important mediators of normal nuclear function and both are disrupted in HGPS. In the first part of this dissertation, I show that the mislocalization of SUMO E2 Ubc9 in HGPS drives the loss of nuclear SUMOylation, which in turn disrupts the Ran gradient. In the second part of this dissertation I demonstrate that a loss of the Ran gradient can also cause defective nuclear import of Ubc9, thus pointing to a mutually regulatory mode of function between the Ran and SUMOylation systems. In addition to causing defective Ubc9 import, disruption of the Ran gradient induces oxidative stress, the latter being a part of a newly emerging concept in HGPS. The resultant increase in ROS could in principle feed back into the Ran gradient generating machinery and worsen the Ran gradient. This study identifies the Ran gradient disruption as an early event in HGPS, which by affecting two diverse pathways namely redox balance and nuclear SUMOylation, serves the role of an important mediator of the progressive cellular changes in HGPS. 


\section{TABLE OF CONTENTS}

Abstract 2

List of Figures $\quad 5$

List of Abbreviations $\quad 8$

$\begin{array}{lr}\text { Publication information } & 10\end{array}$

Chapter one: General introduction 12

The Nuclear Lamina and Nuclear Pore Complexes 12

Nuclear import and Export: The role of Ran 14

$\begin{array}{ll}\text { The SUMOylation machinery } & 17\end{array}$

The Hutchinson-Gilford Progeria Syndrome $\quad 24$

$\begin{array}{ll}\text { Oxidative stress } & 36\end{array}$

Oxidative stress and the nuclear Lamina: the role of prelamin A 40

Oxidative stress and the Ran system 41

Oxidative stress and the SUMOylation machinery 43

Chapter two: The defective SUMOylation machinery in 45 HGPS causes a disruption of the Ran protein gradient.

Summary 45

$\begin{array}{ll}\text { Introduction } & 46\end{array}$

$\begin{array}{ll}\text { Materials and methods } & 48\end{array}$

Results $\quad 52$

$\begin{array}{ll}\text { Discussion } & 72\end{array}$

Chapter three: A central role of the RanGTPase system in the 76 development of SUMOylation defects and oxidative stress in HGPS

Summary $\quad 76$

$\begin{array}{ll}\text { Introduction } & 77\end{array}$

Materials and methods $\quad 81$

$\begin{array}{lr}\text { Results } & 88\end{array}$

$\begin{array}{ll}\text { Discussion } & 133\end{array}$ 
Chapter four: Conclusions and future directions

References 


\section{List of Figures}

Figure 1. Progerin reduces nuclear SUMO2/3 levels.

Figure 2. Inhibition of SUMOylation disrupts the Ran protein gradient.

Figure 3. Reduced nuclear localization of Ubc9 in HGPS.

Figure 4. Transport signal fusions that direct nuclear and cytoplasmic targeting of Ubc9.

Figure 5. Forcing nuclear localization of Ubc9 rescues the Ran gradient in Progerin expressing cells.

Figure 6. Nuclear localization of Ubc9 restores TPR import in cells expressing Progerin.

Figure 7. Nuclear localization of Ubc9 restores H3K9me3 levels in cells expressing Progerin.

Figure 8. Working model for how progerin disrupts the Ran gradient in HGPS.

Figure 9. Progerin inhibits nucleo-cytoplasmic shuttling of the SUMO conjugating enzyme Ubc9.

Figure 10. Heterokaryon cell fusion using Cos cells transfected with HA-Lamin and Flag-Ubc9.

Figure 11. Nuclear import of Ubc9 does not require an interaction with Nup358 at the NPC.

Figure 12. Fibroblasts from HGPS patients have elevated ROS.

Figure 13. Mitochondria from HGPS fibroblasts are depolarized. 
Figure 14. Oxidative stress induced by $\mathrm{H}_{2} \mathrm{O}_{2}$ addition causes Ubc9 re-distribution to the cytoplasm.

Figure 15. Nuclear localization of Ubc9 is resistant to the effects of $\mathrm{H}_{2} \mathrm{O}_{2}$ in $\mathrm{CHO}$ cells pre-adapted to oxidative stress.

106

Figure 16. Stress-resistant $\mathrm{CHO}$ cells resist the effects of Progerin on nuclear transport.

Figure 17. Ubc9 localization to the cytoplasm in response to Progerin and oxidative stress appears to be independent of cysteine 93 oxidation.

Figure 18. Ubc9-Uba2 thioester formation in 8469 fibroblasts.

Figure 19. Nuclear localization of Ubc9 requires a Ran protein gradient.

Figure 20. Lopinavir, a Zmpste24 inhibitor mislocalizes Ran and Ubc9 and elevates ROS levels.

Figure 21. siRNA knockdown of Lamin A prevents LPV induced cellular defects.

Figure 22. Timecourse of prelamin A accumulation, disruption of the Ran gradient and induction of ROS by LPV treatment.

Figure 23. Induction and reversal of cellular defects with LPV treatment and post treatment withdrawal respectively.

Figure 24. ATP measurements with LPV treatment.

Figure 25. Disruption of the Ran protein gradient is sufficient to cause an increase in cellular ROS levels. 
Figure 26. Model showing the proposed pathway of Progerin induced cellular defects.

Figure 27. Model depicting the interdependence of nuclear SUMOylation and the Ran gradient and how it is disrupted in HGPS.

Figure 28. Model demonstrating the possible ways by which Progerin could induce ROS.

Table 1. SENP classification and properties.

20

Table 2. Clinical trials against HGPS. 


\section{List of abbreviations}

CAS Cellular Apoptosis Susceptibility protein

$\mathrm{CHO} \quad$ Chinese Hamster Ovary

DCF 2'7' Dichloro Fluoroscein Diacetate

DMSO Dimethyl Sulfoxide

ECM Extracellular Matrix

EDMD Emery-Dreyfuss Muscular Dystrophy

ER Endoplasmic Reticulum

ETS Electron Transport System

FRAP Fluorescence Recovery After Photobleaching

FTI Farnesyl Transferase Inhibitor

GAP GTPase Activating Protein

GEF Guanine Nucleotide Exchange Factor

H3K27 Lysine 27 of Histone H3

H3K9 Lysine 9 of Histone H3

H4K20 Lysine 20 of Histone H4

HGPS Hutchinson-Gilford Progeria Syndrome

IBB Importin $\beta$ Binding Domain

iPSC Induced Pleuripotent Stem Cell

LPV Lopinavir

MPT Mitochondrial Permeability Transition

N/C Ratio of mean nuclear to cytoplasmic fluorescence 


$\begin{array}{ll}\text { NAC } & \text { N-Acetyl Cysteine } \\ \text { NES } & \text { Nuclear Export Signal } \\ \text { NLS } & \text { Nuclear Localization Signal } \\ \text { NOX } & \text { NADPH Oxidase } \\ \text { NPC } & \text { Nuclear Pore Complex } \\ \text { RCC1 } & \text { Regulator of Chromosome Condensation } \\ \text { RIRR } & \text { ROS induced ROS Release } \\ \text { ROS } & \text { Reactive Oxygen Species } \\ \text { SIM } & \text { SUMO Interaction Motif } \\ \text { SOD } & \text { Superoxide Dismutase } \\ \text { UPR } & \text { Unfolded Protein Response }\end{array}$




\section{Publication information}

The following publication was used as the basis of chapter 2. All figures shown in this chapter were generated by Sutirtha Datta as part of the larger study below.

Kelley, J.B., Datta, S., Snow, C.J., Chatterjee, M., Ni, L., Spencer, A., Yang, C.S., Cubeñas-Potts, C., Matunis, M.J., and Paschal, B.M. The Defective Nuclear Lamina in Hutchinson-Gilford Progeria Syndrome Disrupts the Nucleocytoplasmic Ran Gradient and Inhibits Nuclear Localization of Ubc9. Molecular and Cellular Biology 31, 33783395. 


\section{Acknowledgements and dedication}

I would like to dedicate this work to the children suffering from Progeria and their families, whose courage, determination and positive attitude under these unfortunate circumstances should be viewed as a testimony to the human spirit. This work is also dedicated to all individuals who are associated with the Progeria Research Foundation directly or indirectly and have or will contribute towards its activities, including all academic and non-academic staff. Small contributions fetch big results and in light of the same, I particularly dedicate this work to all researchers who have dedicated their lives in part or in full, to the pursuit of their scientific endeavors. Science answers questions and satisfies our innate curiosities, an attribute that defines us as human, in addition to providing better lives for us.

I would like to thank the Paschal team for providing me with the opportunity to work with them, learn from them and derive inspiration for determining a life goal. Science has never been a trivial pursuit and never will be and without the support of my esteemed colleagues, even this small achievement called $\mathrm{PhD}$ would not have been a possibility. Also deserving mention is the love and support of my family members, who have never questioned my abilities as a scientist and always viewed my pursuits as something valuable. Lastly I would like to express my gratitude for my wife Prianka whose unquestionable faith in my capabilities, even beyond my own, has allowed me to gather the courage and determination to embark on this quest for the unknown. 


\section{Chapter 1 GENERAL INTRODUCTION}

\section{The nuclear lamina and Nuclear Pore Complexes.}

The nuclear lamina is a meshwork of proteins that lines the inner surface of the nuclear envelope (Dechat et al., 2008). Traditionally it has been attributed with the role of providing a structural and supporting framework to the nuclear membrane. However more recently the nuclear lamina has also been implicated in the regulation of gene expression by maintaining the structure and positioning of chromatin (Croft et al., 1999; Sullivan et al., 1999). More specifically the lamina serves as attachment sites for peripheral heterochromatin (Sullivan et al., 1999). It has also been shown to regulate DNA replication, apoptosis and other important physiological processes of the cell (Gruenbaum et al., 2005). The lamina consists of Type $V$ intermediate filament proteins called Lamins along with various other scaffolding proteins that bridge the lamina to chromatin (Gruenbaum et al., 2003). Lamins are subdivided into two types, namely A and $\mathrm{B}$, that are distinguished based on their biochemical properties and their membrane association during Mitosis. Lamin B is essential, ubiquitously expressed and is farnesylated during its maturation, by virtue of which it remains attached to the Endoplasmic Reticulum (ER) and the remnants of the nuclear membrane upon nuclear envelope disassembly during mitosis (Gerace and Blobel, 1980; Gruenbaum et al., 2005). Lamin A on the other hand is only expressed in differentiated cells in a tissue specific manner. It is also farnesylated but the farnesylated portion is subsequently cleaved off, thereby rendering Lamin A soluble during mitosis (Gerace and Blobel, 1980; Gruenbaum et al., 2003). Lamin C arises from alternative splicing of the Lamin A 
gene and is not farnesylated (Gruenbaum et al., 2005). The importance of the nuclear lamina and the degree to which its integrity is required for normal cellular functions, is underscored by the fact that mutations in LMNA, the gene that codes for Lamin A, have been causal to about 12 different diseases termed Laminopathies. These include Emery Dreyfuss Muscular dystrophy (EDMD), dilated cardiomyopathy, atypical Werner's syndrome and Hutchinson Gilford Progeria Syndrome (HGPS) (Mounkes et al., 2003).

Neither the nuclear envelope nor the lamina has a continuous structure. They are studded with giant multimeric complexes of proteins which are arranged in the form of a channel through which the bidirectional process of transport between the nucleus and the cytoplasm can occur (Tran and Wente, 2006). These channels are termed Nuclear Pore Complexes (NPCs) through which molecules up to $40 \mathrm{KDa}$ in size can diffuse passively, while larger molecules undergo the active process of nuclear-cytoplasmic transport (Tran and Wente, 2006). The NPC comprises about 30 proteins called Nucleoporins (Nups) that are arranged in an 8 fold symmetry along the channel (Fahrenkrog and Aebi, 2003). One of the Nups that is localized specifically on the nuclear side of the NPC is TPR, a large protein that assembles to form a basket shaped structure on the nuclear side of the pore (Cordes et al., 1997; Ris, 1989). The nuclear basket has various important functions that include binding of Importin $\beta$ and facilitating the nuclear import of Importin $\beta$ bound cargoes (Ben-Efraim et al., 2009), regulating CRM1 mediated nuclear export (Frosst et al., 2002) and also acting as a scaffold for the localization of key enzymes of the SUMOylation machinery (Hang and Dasso, 2002; Panté et al., 1994; Zhang et al., 2002). 


\section{Nuclear import and export: the role of Ran}

The process of nuclear import begins in the cytosol where an import complex is formed between the cargo and the import receptor(s) by binding of the receptor to stretches of basic amino acid residues on the cargo called Nuclear Localization Signals (NLSs) (Pemberton and Paschal, 2005; Stewart, 2007). NLSs may be monopartite, for example the SV40 Large T antigen NLS (PKKKRKV), bipartite as exemplified by the Nucleoplasmin NLS (KRPAATKKAGQAKKKKL) (Gorlich and Kutay, 1999; Stewart, 2007) as well as various unusual NLSs not conforming to the classical description, many of which are undefined (Pemberton and Paschal, 2005). The classical nuclear import pathway is exemplified by the one involving the Importin $\alpha / \beta$ heterodimer (Stewart, 2007). Typically the NLS binds to ARM repeats on Importin $\alpha$ and the N terminus of Importin $\alpha$ binds to Importin $\beta$ through what is called the Importin $\beta$ binding domain (IBB), thus forming a trimeric cargo-receptor complex. The complex translocates through the central channel of the NPC by virtue of weak hydrophobic interactions between the import receptor and the Phenylalanine Glycine rich motifs (FG motifs) on the Nups that line the inner surface of the NPC (Stewart, 2007). Once the complex reaches the nuclear side of the NPC, the GTP bound form of Ran, a Ras related nuclear protein, binds to Importin $\beta$ and induces a conformational change that dissociates the IBB of Importin $\alpha$, thereby releasing Importin $\beta$ from the complex. The IBB now loops back in an auto-inhibitory fashion and dissociates the NLS from Importin a thereby releasing the cargo (Pemberton and Paschal, 2005). Since the affinity of Importin a for NLSs is high, the off rate is too low to match the rate of transport through the NPC 
(Stewart, 2007). This reaction is favored by certain Nups located on the nucleoplasmic side of the pore. Nup50 for example, a nuclear basket associated Nup, displaces both monopartite and Bipartite NLSs from Importin a by competing with the NLS for Importin $\alpha$, since it has a high affinity for Importin a (Matsuura and Stewart, 2005). Nup153 on the other hand assists the release of Importin $\beta$ cargoes by acting as a local reservoir of RanGTP which can dissociate the complex (Schrader et al., 2008). A high concentration of RanGTP is maintained in the nucleus by the activity of RCC1, a chromatin bound enzyme that catalyzes GDP-GTP exchange on Ran (Bischoff and Ponstingl, 1991; Ohtsubo et al., 1989). RCC1's catalytic cycle is closely coupled to its on and off rate on chromatin and is stimulated by binding to histones H2A and H2B (Li et al., 2003; Nemergut et al., 2001). Following dissociation of the import complex and the release of the cargo, the import receptor(s) can either translocate back to the cytosol in a complex with RanGTP (eg. Importin $\beta$ ) or can be exported as a complex between RanGTP and an export factor CAS (Cellular Apoptosis Susceptibility protein) (eg. Importin $\alpha$ ) (Stewart, 2007). Typically protein export cargo complexes are formed between RanGTP, Leucine rich stretches on the cargo termed the Nuclear Export Signal (NES) and export receptor CRM1 (Fornerod et al., 1997; Fukuda et al., 1997; Pemberton and Paschal, 2005). After translocation of the export complex through the NPC to the cytosol, the NPC bound RanGTPase activating protein (RanGAP) along with the assistance of Nup358 and Ran Binding Protein 1 (RanBP1), catalyzes the hydrolysis of RanGTP to RanGDP. The conformational change driven by this event, is sufficient to dissociate the export complex and release the cargo in the cytoplasm, following which CRM1 can shuttle back into the nucleus (Bischoff et al., 1995; Bischoff et al., 1994b). 
Nup358 has very important roles to play in the coupling of nuclear import and export (Melchior, 1995; Yaseen and Blobel, 1999). It was discovered as a large $358 \mathrm{KD}$ protein with a putative RanGTP binding domain and FG repeats characteristic of nucleoporins (Wu et al., 1995). By immunogold electron microscopy, it is localized onto filaments of the NPC emanating on the cytosolic side (Wu et al., 1995). Nup358 was shown to bind Ran GTP and serve as a docking site where Ran GTP hydrolysis can allow Ran GDP to bind with the Importin-cargo complex (Melchior, 1995; Yaseen and Blobel, 1999). Whether Nup358 is necessary for Importin $\alpha / \beta$ dependent Import, is disputed (Hutten et al., 2008; Walther et al., 2002). However its role in CRM1 mediated export is well documented. It serves as a platform for disassembly of export complexes once translocated through the pore to the cytosol and a binding site for the recycling of empty CRM1 into the nucleus (Bernad et al., 2004; Engelsma et al., 2004).

Since in an import-export cycle, Ran does not enter the nucleus, but can exit in multiple ways (in complex with the import receptors as well as with the export cargo), there is a deficit of Ran in the nucleus after one cycle. This deficit is fulfilled by Nuclear Transport Factor 2 (NTF2) (Paschal and Gerace, 1995), a shuttling receptor for Ran that binds only to the GDP bound form of Ran and carries it to the nucleus through the NPC (Ribbeck et al., 1998; Smith et al., 1998b). Inside the nucleus the conversion of RanGDP to Ran GTP by RCC1 allows the dissociation of Ran from NTF2, following which NTF2 can shuttle back to the cytoplasm (Ribbeck et al., 1998; Smith et al., 1998b). The NTF2 mediated enrichment of Ran in the nucleus coupled with the activities of chromatin bound RCC1 and the cytoplasm localized RanGAP, lead to the generation of two nuclear to cytoplasmic gradients of Ran: the extremely steep RanGTP 
gradient (500:1) and the Ran protein gradient (3:1) under steady state conditions (Gorlich et al., 2003; Kelley and Paschal, 2007). By default there is no directional selectivity to the translocation of import or export complexes through the NPC. Import and export cargo can move in either direction (Stewart, 2007). However the dissociation of the import complex in the nucleus by RanGTP, and of the export complex by RanGAP in the cytosolic side of the NPC allows the release of import cargo in the nucleus and the export cargo in the cytoplasm. Thus during interphase, the role of the Ran gradient is to provide directionality to the process of import and export (Stewart, 2007). Ran has other roles during Mitosis, namely the nucleation of microtubules and reformation of the nuclear envelope (Hetzer et al., 2002). It does so by releasing nucleation factors from their inactive complexes with Importin $\beta$ and by regulating the first steps of membrane fusion during nuclear envelope re-assembly, respectively (Hetzer et al., 2002).

\section{The SUMOylation machinery}

Biochemical analysis of RanGAP revealed that it is covalently modified by SUMO, a Small Ubiquitin related Modifier that shares structural but not a significant degree of sequence homology with Ubiquitin (Bayer et al., 1998; Geiss-Friedlander and Melchior, 2007; Mahajan et al., 1997; Matunis et al., 1996). There are four paralogs of SUMO namely SUMO1, SUMO2, SUMO3 and SUMO4. SUMO 1 has $~ 50 \%$ identity to SUMO2 and SUMO2 and 3 share 97\% identity with each other and hence grouped together (Geiss-Friedlander and Melchior, 2007). SUMO 1, 2 and 3 are ubiquitously expressed 
while SUMO4 is only found in the Kidney, lymph node and spleen (Guo et al., 2004). Biochemical analyses of differences between the behavior of SUMO1 and SUMO2/3 revealed that SUMO2/3 is more abundant in its free form in resting cells, but under various stress conditions, SUMO2/3 conjugation can increase dramatically. On the other hand SUMO1 is mostly conjugated to RanGAP and is less dynamic compared to SUMO2/3 upon the induction of various cellular stresses (Saitoh and Hinchey, 2000). SUMO modification of proteins can have a multitude of effects. It can alter the subcellular localization of proteins, as in the case of Bovine Papillomavirus E1 helicase, a protein responsible for the replication of Bovine Papillomavirus. This protein is SUMOylated at a single lysine residue, and point mutations that abolish SUMOylation also inhibit its nuclear import (Rangasamy et al., 2000). A second prominent example would be that of RanGAP which is soluble in the cytosol when unmodified, but targets to the NPC only when modified by SUMO1 (Matunis et al., 1996). SUMOylation can alter the interaction patterns of proteins. Ubc9 the SUMO E2 when auto-SUMOylated on Lys 14, has altered activity towards different substrates based on its differential binding interactions with the substrate (Knipscheer et al., 2008). Another classic example is that of chromatin binding proteins. P300 is a transcriptional coactivator when unmodified whereas upon SUMOylation, it acts as a co-repressor by recruiting Histone Deacetylases (HDACs), leading to a local compaction of chromatin (Girdwood et al., 2003). SUMOylation also regulates the stability of proteins as exemplified by $I \kappa B \alpha$, an inhibitor of NFKB. Upon phosphorylation of $\mathrm{IKBa}$, it is ubiquitinated and degraded, allowing the release of NFKB (Brown et al., 1995). Interestingly SUMO1 modification of IKBa at the same residue that is ubiquitinated, antagonizes ubiquitination and prevents 
its degradation (Desterro et al., 1998). Likewise many other functions can be attributed to SUMO modifications and the list is rapidly growing.

The SUMO modification cycle begins with the processing of the SUMO peptide at its C terminal end by SUMO proteases or SENPs (Mukhopadhyay and Dasso, 2007). SENPs are classified into various types (1 through 7) based on their subcellular localization and paralog specific activity (Mukhopadhyay and Dasso, 2007; Shen et al., 2009) (Table1). Following their processing by SENPs, the SUMO peptide is activated by an ATP dependent reaction, leading to a thioester formation with the SUMO E1 complex, a heterodimer of AOS1 and Uba2. The thioester is formed between a catalytic cysteine on Uba2 and the C terminal glycine on SUMO (Geiss-Friedlander and Melchior, 2007). The SUMO E1 complex then transfers the SUMO peptide to the active site of the SUMO E2 Ubc9, the only known E2 ligase, where a thioester is formed between SUMO and C93 on Ubc9. In a final catalytic step, Ubc9 carries out the covalent attachment of SUMO to target Lysines on the substrate. More specifically, an isopeptide bond is formed between the C terminal Glycine residue of SUMO and an $\epsilon$ Lysine residue of the target. The consensus target sequence is $\psi \mathrm{KxE}$, where $\psi$ is a branched aliphatic amino acid and $\mathrm{x}$ is any amino acid (Geiss-Friedlander and Melchior, 2007). The final catalytic step by Ubc9 is assisted by a family of SUMO E3 ligases that confer target specificity by serving as a scaffold for the favorable orientation of Ubc9 and the target being modified (Geiss-Friedlander and Melchior, 2007). Some of the common E3 ligases include the PIAS family, the polycomb protein pc2 and also the NPC associated nucleoporin Nup358 or RanBP2, although recently it has been shown that the whole multimer of SUMO1 modified RanGAP, Nup358 and Ubc9 serves as a 
Table 1

SENP classification and properties

\begin{tabular}{|l|l|l|}
\hline Enzyme & Subcellular localization & Paralog specificity \\
\hline SENP1 & $\begin{array}{l}\text { Nuclear Pore, } \\
\text { Nucleoplasmic speckles }\end{array}$ & SUMO-1 and SUMO-2/3 \\
\hline SENP2 & Nuclear Pore & SUMO-1 and SUMO-2/3 \\
\hline SENP3 & Nucleolus & SUMO-2/3 \\
\hline SENP5 & Nucleolus & SUMO-2/3 \\
\hline SENP6 & Nucleoplasm & SUMO-2/3 \\
\hline SENP7 & Nucleoplasm & SUMO-2/3 \\
\hline
\end{tabular}


composite E3 ligase rather than RanBP2 alone being the E3 (Johnson and Gupta, 2001; Kagey et al., 2003; Pichler et al., 2002; Werner et al., 2012). SUMO modification is a reversible process. The same family of SUMO proteases called SENPs that process SUMO, can reverse a SUMO modification and this activity is also paralog specific (Geiss-Friedlander and Melchior, 2007). The existence of separate SUMOylating and de-SUMOylating enzymes allows the SUMOylation process to be dynamically regulated. This comes in handy in situations where the SUMOylated vs deSUMOylated state of a protein has different affinities for the same binding site and hence may contribute towards the binding and dissociation kinetics. This is exemplified by Thymidine DNA Glycosylase (TDG) that executes Nucleotide Excision Repair (Hardeland et al., 2001). TDG when unmodified has a high affinity for DNA and binds stably to chromatin. Upon SUMOylation, its affinity for chromatin decreases and it comes off, only to be de-SUMOylated by SENPs and bind to chromatin again (Hardeland et al., 2002). Thus the catalytic cycle of enzymes can be dynamically regulated by SUMOylation and de-SUMOylation.

The SUMO E2 Ubc9 was discovered in Xenopus extracts during a screen for Ran binding proteins (Saitoh et al., 1997). It was found to coimmunoprecipitate with a complex of Nup358/RanBP2 and RanGAP and was designated p18. Based on its sequence homology to Ubiquitin ligases, it was initially believed to be a Ubiquitin conjugating enzyme (Saitoh et al., 1997). Later on its activity was demonstrated to be specific to SUMO and not Ubiquitin (Lee et al., 1998). By immunofluorescence microscopy, Ubc9 localizes to the nucleoplasm, cytoplasm and also at the NPC, in association with SUMO1 modified RanGAP and Nup358 (Lee et al., 1998; Zhang et al., 
2002). The crystal structure of this complex shows Ubc9 to be centrally located, with Nup358 and SUMOylated RanGAP on either side (Reverter and Lima, 2005). The Ubc9 binding domain on Nup358 is called the IR domain which consists of IR1, M and IR2 domains (Pichler et al., 2002). Overexpression of IR $1+2$ domains is sufficient to mislocalize nucleoplasmic Ubc9 into the cytosol without affecting the NPC associated Ubc9 (Saitoh et al., 2002). This suggests that the Nup358 associated pool of Ubc9 and the nucleoplasmic pool could be independent of each other for their subcellular localization. By immunogold electron microscopy, Ubc9 was also shown to reside on the nuclear side of the NPC, however the significance of this particular pool of Ubc9 has not been documented (Zhang et al., 2002). Ubc9 is imported into the nucleus by an Importin $\beta$ family member Importin13 (Mingot et al., 2001a). Upon translocating through the NPC, the Ubc9-Importin13 complex is dissociated by RanGTP by virtue of competition between RanGTP and Ubc9 for the overlapping binding sites on Importin13 (Grunwald and Bono).

Although most targets of Ubc9 are nuclear, SUMOylation occurs in the cytoplasm as well (Geiss-Friedlander and Melchior, 2007). Various plasma membrane localized ion channels have been shown to be SUMO modified and this has been linked to their activities or internalization patterns (Geiss-Friedlander and Melchior, 2007). SUMOylation has also been shown to regulate mitochondrial dynamics or the activities of proteins that are tethered to the ER membrane facing the cytosol (Geiss-Friedlander and Melchior, 2007). These SUMOylation events could be attributed to the cytosolic pool of Ubc9 although further evidence is required. Particularly interesting is the localization of Ubc9 to the Cytosolic side of the NPC coupled with the fact that SENP2 
docks on to Nup153 on the nuclear side of the pore (Lee et al., 1998; Zhang et al., 2002). The presence of a SUMO modifying-demodifying pair on either side of the NPC, hints towards the possibility that for certain cargoes, the process of nuclear transport could be closely coupled to their SUMOylation state. SUMOylation by Ubc9 on the cytosolic side of the pore could commit them for nuclear transport and their chances of diffusing back out of the nucleus could be reduced by de-SUMOylation on the nuclear side. In fact, the nuclear distribution of various proteins have been shown to be regulated by their SUMOylation on specific Lysine residues and SUMOylation defective mutants fail to undergo nuclear import (Pichler and Melchior, 2002; Rangasamy et al., 2000).

A relatively recent contribution to the SUMOylation field has been that of noncovalent SUMO interactions (Geiss-Friedlander and Melchior, 2007). Proteins recognizing and binding to SUMO non-covalently, do so with SUMO Interaction motifs (SIMs). These are stretches of hydrophobic residues $(\mathrm{V} / \mathrm{I}-\mathrm{x}-\mathrm{V} / \mathrm{I}-\mathrm{V} / \mathrm{I})$ or $(\mathrm{V} / \mathrm{I}-\mathrm{V} / \mathrm{I}-\mathrm{x}-\mathrm{V} / \mathrm{I})$, where $x$ can be any amino acid. These are arranged in the form of a hydrophobic core, flanked by acidic residues (Song et al., 2005). The interaction between a SIM and SUMO is mediated by the second $\beta$ sheet and the first $\alpha$ helix of SUMO. The binding is stabilized by an interaction between the acidic residues flanking the SIM and basic residues on SUMO (Song et al., 2005). SIMs are present on many SUMO substrates and are believed to have a role in the recognition and recruitment of SUMO loaded Ubc9 to the target by virtue of non-covalent interactions, thereby facilitating SUMOylation of the target (Lin et al., 2006; Zhu et al., 2008). SIM-SUMO interactions have also been shown to regulate the formation of multimeric complexes as is the case 
with PML nuclear bodies (Dellaire and Bazett-Jones, 2004). PML is SUMOylated and other PML molecules that recognize and bind to the SUMO moiety through interactions with their SIMs, are recruited to the complex and this forms the basis of the nucleation step that allows the formation of PML nuclear bodies (Shen et al., 2006). Thus the induction of conformational changes in proteins by SUMO modification and the non covalent recognition of these SUMO moieties by other proteins containing SIMs, forms the basis of how SUMOylation can lead to altered protein-protein interactions and contribute significantly towards a large variety of fundamental processes within the cell. This, coupled with the fact that the SUMO modification is reversible, brings SUMO to the forefront of modifications that can dynamically regulate protein function.

\section{The Hutchinson Gilford Progeria Syndrome}

The Hutchinson Gilford Progeria syndrome (HGPS) is a premature aging syndrome in children that is caused by a mutation in the Lamin A gene (De SandreGiovannoli et al., 2003; Eriksson et al., 2003a). The children appear normal at birth but within a year, they show increased signs of aging that include loss of body fat, muscle wasting, stiffening of joints, loss of body hair and various cardiovascular complications (Hennekam, 2006). The average lifespan of a Progeria patient is 13 years and death usually occurs by heart attack or stroke (Capell et al., 2007). The molecular basis of the disease appears to be an extremely rare C1824T mutation near the $C$ terminus of Lamin A which leads to a silent mutation G608G at the protein level. This point mutation however leads to the activation of a cryptic splice site within exon 11 of Lamin A, due to 
which 50 amino acids are absent from Lamin A (De Sandre-Giovannoli et al., 2003; Eriksson et al., 2003a). During the normal maturation of Lamin A, a precursor of Lamin A is synthesized, which is designated prelamin A (Gerace and Benson, 1984). Prelamin A undergoes a series of modification steps. First it is Farnesylated at its $C$ terminal CaaX motif by the activity of Farnesyl transferases. This attaches a lipid anchor to prelamin A by virtue of which it can insert into the nuclear membrane following its import into the nucleus (Holtz et al., 1989). Following farnesylation and targeting to the nuclear membrane, the aaX motif is cleaved off by a Zinc Metalloprotease related to Ste24p (Zmpste24) and RCE1 and the terminal cysteine is carboxymethylated by the methyltransferase ICMT (Dechat et al., 2008). The final maturation step constitutes a second cleavage by Zmpste24 such that the short $\mathrm{C}$ terminal segment containing the farnesylation, is cleaved off and mature Lamin A that is without any lipid anchor, is released (Corrigan et al., 2005; Weber et al., 1989). These maturation steps were originally believed to occur in the cytoplasm and the Lamin A processing enzymes were shown to reside in the ER membrane with their active sites facing the cytosol (Dai et al., 1998; Schmidt et al., 1998). However recently, this concept was revised as the same Lamin A processing enzymes were also shown to reside in the inner nuclear membrane with their catalytic sites facing the nucleoplasm (Barrowman et al., 2008). In HGPS, as a result of the 50 amino acid deletion, the second Zmpste24 cleavage site is lost. Therefore mature Progerin still retains the farnesyl group, remains attached to the nuclear membrane and drives its pathogenic phenotypes (De Sandre-Giovannoli et al., 2003; Eriksson et al., 2003a). 
The cellular phenotyes associated with HGPS are many and these are mostly manifested at late passage cells in culture when the expression of Progerin is maximal (McClintock et al., 2006; Rodriguez et al., 2009). The most characteristic of the phenotypes, is the distortion of the nuclei, a term called 'Blebbing' (Eriksson et al., 2003a; Goldman et al., 2004). This is likely caused by the insertion of Progerin into the lamina and a defective polymerization of the lamin network via a dominant negative effect of Progerin (Dahl et al., 2006). Indeed Fluorescence Recovery After Photobleaching (FRAP) studies of nuclei containing WT Lamin and Progerin revealed that Progerin reduces the mobility of the lamins drastically. Upon overexpression, Progerin has much slower recovery kinetics upon photobleaching, compared to wild type Lamin A (Dahl et al., 2006; Goldman et al., 2004). Even WT Lamin A when overexpressed in HGPS cells displays reduced mobility by FRAP analysis, indicating the dominant negative effect of Progerin (Dahl et al., 2006; Scaffidi and Misteli, 2005a). The reduced mobility of Progerin compared to WT Lamin A was also verified by salt extraction studies (Dahl et al., 2006). The biomechanical properties of Progerin containing nuclei are very different from normal nuclei, in that the former are more rigid and less potent for recovery to their original structure after deformation (Dahl et al., 2006). This is due to a more ordered microdomain structure of the lamina in Progerin containing nuclei as opposed to a completely disordered form in normal nuclei. In other words, Progerin containing nuclei are less resistant to mechanical stress (Dahl et al., 2006). SUN1, a component of the LINC complex that bridges the lamina to the Actin cytoskeleton, has been shown to increase its association at the lamina in HGPS cells (Haque et al., 2009). This is hypothesized as being the result of increased association 
of SUN1 with prelamin A in comparison to Lamin A. Since Progerin is similar to prelamin A in terms of its membrane association properties, it could be directly responsible for the enhanced recruitment of SUN1 to the nuclear envelope. Since the coupling between the lamina and the cytoskeleton has a role in regulating the mechanical properties of the nucleus, this could be an additional means for the increased mechanical stiffness of HGPS nuclei (Haque et al., 2009). Interestingly, SUN1 has been implicated in the pathogenicity of HGPS because knockdown of SUN1 in mouse models of HGPS, alleviates many of the nuclear defects, increases longevity and reduces cellular senescence in HGPS mice (Chen et al., 2012). Lamin B is mislocalized in HGPS fibroblasts and both Lamina Associated Polypeptide 2 (LAP2) and ING1, proteins that bridge the lamina with chromatin, no longer associate with the lamina (Han et al., 2008; Scaffidi and Misteli, 2005a, 2006). This provides further evidence that the general structure of the lamina is perturbed by the dominant negative effects of Progerin.

The mislocalization of proteins bridging the lamina-chromatin interface is expected to perturb the structure of chromatin. Indeed there are gross changes in the chromatin structure. Peripheral heterochromatin that associates with the lamina, is lost in HGPS nuclei (Goldman et al., 2004). This is backed by the observation that both $\mathrm{H} 3 \mathrm{~K} 9$ and H3K27 trimethylation (repressive modifications) are reduced and H4K20 trimethylation (activating modification) is increased in fibroblasts from HGPS patients (Scaffidi and Misteli, 2006; Shumaker et al., 2006b). Thus on a global level, the chromatin from HGPS patient cells is in a more open conformation. As a result, there is an alteration in the global patterns of gene expression. Moreover since a lot of genes are silenced by physical association with the nuclear lamina (Reddy et al., 2008), the 
disruption of the lamina by Progerin expression is likely to produce an aberrant profile of gene expression. The gene expression changes associated with HGPS are summarized in (Prokocimer et al., 2013). The results of one such study show that the expression of various transcription factors responsible for regulating embryonic development and tissue differentiation is markedly repressed in HGPS (Csoka et al., 2004). Some of the dysregulated gene groups are also implicated in atheroschlerosis, indicating that this may be involved in producing the cardiovascular abnormalities in HGPS. This study also reveals striking differences in the expression levels of genes encoding Extra Cellular Matrix $(\mathrm{ECM})$ components and the genes that regulate the differentiation of Mesodermal and Mesenchymal stem cells (Csoka et al., 2004). The results of the gene expression profiling analysis with respect to ECM components and Mesenchymal stem cell differentiation factors, were also corroborated in cellular and organismal level studies. Postnatal fibroblasts from mice homozygous for $\operatorname{Lmna} \Delta 9$, that mimic many of the pathological developments of HGPS, are defective for synthesis and secretion of ECM components and this is caused by an inhibition of the canonical Wnt signaling pathway (Hernandez et al., 2010). Furthermore Progerin expression was shown to inhibit the differentiation potential of mesenchymal stem cells and this was shown to be transduced through aberrant constitutively active Notch signaling (Scaffidi and Misteli, 2008). The Notch activator Ski interacting protein (SKIP) is normally repressed via its attachment to the lamina (Scaffidi and Misteli, 2008). Upon Progerin expression, SKIP is freed from the lamina, diffuses into the nucleus and aberrantly turns on the expression of Notch target genes. As a result, the normal differentiation program of mesenchymal stem cells is disrupted, leading to increased Osteogenesis at the cost of adipogenesis 
(Scaffidi and Misteli, 2008). In another study Zmpste24 ${ }^{-}$mice, which mimic the HGPS mutation were shown to have defects in both the self-renewal as well as differentiation potential of Hair follicle stem cells and this was attributed to a disrupted Wnt signaling pathway (Espada et al., 2008). An incredibly powerful approach was the somatic reprogramming of HGPS dermal fibroblasts to form inducible pluripotent stem cells (iPSCs) (Liu et al., 2011). Smooth muscle cells derived from these iPSCs suffered from premature senescence, nuclear morphology defects and loss of H3K9me3 levels (Liu et al., 2011). iPSCs derived from HGPS dermal fibroblasts, when differentiated into mesenchymal stem cells and vascular smooth muscle cells, also showed defects in the viability and oxidative stress resistance of the resultant stem cells (Zhang et al., 2011). Interestingly skin biopsies from HGPS patients show maximal Progerin expression in vascular smooth muscle and endothelial cells (McClintock et al., 2006). The same iPSCs when differentiated into adipocytes revealed a defective adipogenic gene expression program vis-à-vis commitment to later adipogenic lineages (Xiong et al., 2013). The above observations subscribe to a stem cell defect model of HGPS, wherein the defective function of stem cells within the tissues of HGPS patients, leads to an inability to replenish damaged tissue, thereby culminating in massive net tissue destruction in HGPS (Meshorer and Gruenbaum, 2008). Interestingly adult stem cells derived from HGPS patients have been shown to express low levels of Progerin in vivo (Wenzel et al., 2012).

The signaling defects in HGPS are not just restricted to the Wnt and Notch pathways. A major signaling defect has also been demonstrated to occur in the DNA damage response pathways in HGPS (Liu et al., 2005; Scaffidi and Misteli, 2006); (Liu 
et al., 2006); (Liu et al., 2008). The DNA damage response is initiated by the activation of the kinases Ataxia-Telengiectasia Mutated (ATM) and ATM and Rad3 related (ATR) that phosphorylate and activate their targets Chk2 and Chk1 respectively. Chk1 and Chk2 phosphorylates and activates p53. As a result a DNA damage specific transcription program is initiated by p53, that drives cell cycle arrest (Zhou and Elledge, 2000). The repair pathway comprises the activation of the kinase DNA-PK, which along with ATM and ATR, phosphorylates histone H2AX at the sites of DNA damage, thereby marking the sites where the repair machinery needs to be targeted. This appears in the form of $\mathrm{\gamma H}_{2} \mathrm{AX}$ foci in the nuclei (Kinner et al., 2008). Following this initial marking of the damage sites, p53 binding protein 1 (53BP1) is recruited to the H2AX phosphorylation sites (Schultz et al., 2000). In a series of subsequent recruitment steps, DNA repair proteins are recruited and engaged in repairing the damaged ends of DNA (Petrini and Stracker, 2003). After a successful repair of the damaged DNA, the $\mathrm{\gamma H} 2 \mathrm{AX}$ foci slowly disappear, the rate of disappearance being directly proportional to the efficacy of the repair process (Rogakou et al., 1999). Fibroblasts from HGPS patients have increased accumulation of damaged DNA as demonstrated by the increased persistence of $\gamma \mathrm{H} 2 \mathrm{AX}$ foci and these do not co-localize with 53BP foci, indicating that the repair pathways are not functional (Liu et al., 2005; Scaffidi and Misteli, 2006). There is an increased activation of ATM and ATR kinases and both Chk1 and Chk2 are phosphorylated in cells from HGPS patients (Liu et al., 2006). Upon the infliction of DNA damage by UV irradiation, HGPS cells have a much slower recovery rate compared to normal cells, indicative of defective DNA damage repair mechanisms (Liu et al., 2005). In fact the recruitment of the DNA repair proteins Rad50 and Rad51 to the DNA damage 
sites is also impaired in HGPS cells (Liu et al., 2008). In agreement with the fact that activated p53 signaling pathways during DNA damage induces cell cycle arrest, HGPS cells have an extremely slow rate of proliferation in culture (Bridger and Kill, 2004). This replicative senescence effect in culture is accompanied by shortening of Telomeres in HGPS fibroblasts (Allsopp et al., 1992; Benson et al., 2010; Cao et al., 2011a; Huang et al., 2008). In the event of failed or insufficient DNA damage repair, p53 activates an apoptotic program that aims to eliminate cells containing damaged DNA from the population (Zhou and Elledge, 2000). Indeed this is applicable to HGPS fibroblasts in culture wherein they have a larger population of actively dividing cells compared to normal fibroblasts during early passages (Bridger and Kill, 2004). However, compared to the gradual decline in actively dividing cells, as is the case with normal fibroblasts, HGPS fibroblasts have a rapid decline in their actively dividing population, and this is attributed to the presence of a large percentage of apoptotic cells (Bridger and Kill, 2004).

In addition to cell cycle defects, HGPS cells have abnormalities in chromosome segregation and cytokinesis which shown to be a consequence of the membrane association of Progerin throughout the duration of Mitosis (Cao et al., 2007; Dechat et al., 2007; Goldman et al., 2004). Normally by late prophase, after the breakdown of the nuclear envelope into vesicles, Lamin A has a completely diffuse distribution in the cell although Lamin B should still be associated with the nuclear membrane vesicles (Gerace and Blobel, 1980; Moir et al., 2000). Interestingly in the case of HGPS, Progerin was shown to form discrete foci throughout the duration of Metaphase to Telophase, even during cytokinesis when WT Lamin A had assembled into the lamina 
(Cao et al., 2007; Dechat et al., 2007; Goldman et al., 2004). These foci were shown to be Progerin aggregating at the nuclear membrane vesicles, which interfered with the recruitment of Lamin A, Lamin B and Emerin to the nuclear membrane and the reassembly of the lamina (Cao et al., 2007; Dechat et al., 2007). As a consequence, HGPS cells have delays in Mitosis and lead to the formation of binucleate cells (Cao et al., 2007; Dechat et al., 2007).

Therapeutic strategies for the reversal of the cellular defects in HGPS are already under way. Using a morpholino oligonucleotide that specifically targets the cryptic splice site activated in HGPS, the aberrant expression of Progerin was blocked and this was sufficient to reverse some of the cellular phenotypes of HGPS including the mislocalization of LAP2 and Lamin B, loss of heterochromatin, as well as restore the expression patterns of a few chosen genes that are misregulated in HGPS (Scaffidi and Misteli, 2005b). A similar oligo also increased body weight, thickened the layer of subcutaneous fat, reduced senescence markers and significantly increased lifespan in a mouse model of HGPS (Osorio et al., 2011). Using shRNA targeted against Progerin, another group successfully reduced Progerin levels in HGPS fibroblasts and achieved an improvement in nuclear morphology, cell viability and a reduction in the number of senescent cells (Huang et al., 2005).

Another successful strategy involved the use of Farnesyl Transferase Inhibitors (FTIs) that block the farnesylation of Progerin and prevent its association with the nuclear membrane. This was sufficient to reverse the nuclear morphology defects in cultured cells as well as improve bone density, body weight and survival in mouse models (Capell et al., 2005; Fong et al., 2006; Glynn and Glover, 2005; Mallampalli et 
al., 2005; Toth et al., 2005; Yang et al., 2006). A recent contribution to the plethora of cellular defects in HGPS, was the finding that the Ran protein gradient is disrupted in fibroblasts from HGPS patients, with a concomitant loss of nuclear SUMOylation (Kelley et al., 2011). The loss of the Ran gradient also leads to defective import of nucleoporin TPR into the nucleus, and all of these defects are reversed by FTI treatment (Kelley et al., 2011). The possibility of alternative prenylation of Lamin A in the absence of farnesylation led to the combined usage of Statins and aminobisphosphonates. These drugs block geranylgeranylation of Lamin A and they significantly improved growth, increased body fat and extended lifespan in Zmpste24 ${ }^{-/}$mouse models of HGPS (Varela et al., 2008). Based on the success obtained in mouse models with FTIs and Statins-bisphosphonates, clinical trials were conducted in HGPS patients, a summary of which is shown in Table 2 (Gordon et al., 2012) (Varela et al., 2008) (Clinical trials.gov).

Four other recent discoveries also hold promise in the design of a possible therapeutic intervention. Firstly treating Zmpste24 ${ }^{-/-}$mice with Insulin like growth factor 1 (IGF-1) increased body weight and significantly increased life span in addition to ameliorating other Progeria phenotypes (Mariño et al., 2010). The second is the finding that the SUN proteins, specifically SUN1 have a pathogenic role in the propagation of HGPS. SUN1 mislocalizes to the golgi in LMNA ${ }^{-1}$ and LMNA $\triangle 9$ mice and causes nuclear herniations similar to those observed in HGPS, along with various progeroid symptoms at the tissue level (Chen et al., 2012). SUN1 is also mislocalized in HGPS fibroblasts and this correlates with nuclear $\mathrm{H} 3 \mathrm{~K} 9 \mathrm{me} 3$ reduction as well as the incidence of dysmorphic nuclei. Mice lacking SUN1 have an alleviation of many of these phenotypes. Knocking down SUN1 in HGPS cells also reversed some of the cellular 
Table 2

Clinical trials against HGPS

\begin{tabular}{|c|l|c|l|l|c|l|}
\hline Drug & Class & $\begin{array}{l}\text { Study } \\
\text { phase }\end{array}$ & Dose & Date & $\begin{array}{l}\text { No. of } \\
\text { children }\end{array}$ & \multicolumn{1}{|c|}{ Results } \\
\hline Lonafarnib & $\begin{array}{l}\text { Farnesyl } \\
\text { transferase } \\
\text { inhibitors }\end{array}$ & II & $\begin{array}{l}150 \\
\mathrm{mg} / \\
\mathrm{m}^{2}\end{array}$ & $\begin{array}{l}\text { May } \\
2007- \\
\text { Oct } \\
2009\end{array}$ & 25 & $\begin{array}{l}\text { Weight gain, improved } \\
\text { hearing, improved } \\
\text { bone density in a } \\
\text { subset of patients. }\end{array}$ \\
\hline $\begin{array}{l}\text { Pravastatin, } \\
\begin{array}{l}\text { Zoledronic } \\
\text { acid }\end{array}\end{array}$ & $\begin{array}{l}\text { Geranylger } \\
\text { anyl } \\
\text { transferase } \\
\text { inhibitors }\end{array}$ & $\mathrm{II}$ & $\begin{array}{l}10 \\
\mathrm{mg}, \\
12.5 \\
\mu \mathrm{g} / \mathrm{kg}\end{array}$ & $\begin{array}{l}\text { Oct } \\
2008-\end{array}$ & - & Not available \\
\hline
\end{tabular}


defects (Chen et al., 2012). Thirdly Rapamycin, an inhibitor of MTOR, a component of the TOR signling pathway, was shown to increase the solubility of Progerin and thereby prevent its membrane association (Cao et al., 2011b). It triggered the clearance of Progerin by autophagic mechanisms and successfully reversed nuclear blebbing, the loss of heterochromatin marks. It also reduced the amount of DNA damage and delayed senescence of HGPS fibroblasts (Cao et al., 2011b). The fourth is the very recent finding that targeting isoprenylcysteine methylation in a Zmpste24 mouse model of HGPS by making a hypomorphic allele of Isoprenylcysteine carboxyl methyltransferase (ICMT), prevents the full processing of Lamin A and generates prelamin A that is defective in its targeting to the nuclear envelope (Ibrahim et al., 2013). This manipulation improved weight gain and grip strength, reduced bone fractures and ameliorated many of the other physiological symptoms of premature aging. It also delayed premature senescence in Zmpste24 ${ }^{-/}$as well as HGPS fibroblasts (Ibrahim et al., 2013).

A fairly current addition to the list of defects in HGPS is the increased accumulation of Reactive Oxygen Species (ROS). Oxidative stress is one of the hallmarks of aging, and being a premature aging syndrome, HGPS is likely to involve the same. Fibroblasts from HGPS patients not only have increased basal levels of ROS compared to normal fibroblasts, but also have much higher levels of ROS induced by $\mathrm{H}_{2} \mathrm{O}_{2}$ treatment (Pekovic et al., 2011; Richards et al., 2011; Viteri et al., 2010). Oxidative stress in HGPS is responsible at least in part, for the accumulation of damaged DNA because the degree of DNA damage can be moderated by the use of redox scavengers like N-Acetyl Cysteine(NAC) (Richards et al., 2011). 


\section{Oxidative stress}

ROS are byproducts of normal metabolism and constitute a diverse family of chemical species including $\mathrm{H}_{2} \mathrm{O}_{2}$, Superoxide anions $\left(\mathrm{O}_{2}^{--}\right)$, Hydroxyl radicals, etc. These are highly reactive entities that cause oxidative damage to proteins and lipids by irreversibly modifying them (Finkel and Holbrook, 2000). This is the basis of the free radical theory of aging according to which the degree of cumulative cellular damage by oxidative stress, negatively correlates with the overall longevity of the organism (Finkel and Holbrook, 2000). ROS are mostly produced at complex III of the Mitochondria although Complex I has also been implicated (Finkel and Holbrook, 2000). During the transfer of electrons to molecular Oxygen as a part of the Electron Transport System (ETS) in the inner membrane of the Mitochondria, the occasional premature leakage of electrons before the final step catalyzed at Complex IV, is considered to be the source of ROS (Finkel and Holbrook, 2000). At complex III or Ubiquinone-Cytochrome C oxidoreductase, there is a two-step regeneration of Coenzyme $Q$ involving what is termed the Q cycle (Finkel and Holbrook, 2000). As an intermediate in this cycle is the highly reactive semiquinone radical which can transfer electrons directly to molecular Oxygen leading to superoxide production (Finkel and Holbrook, 2000). This highly unstable species is dismutated by mitochondrial Superoxide dismutase (SOD) to form $\mathrm{H}_{2} \mathrm{O}_{2}$ which is a more stable and diffusible intermediate, that in small concentrations plays a role in modulating various signaling events in the cell (D'Autréaux and Toledano, 2007). At higher concentrations, $\mathrm{H}_{2} \mathrm{O}_{2}$ can have damaging consequences on cellular components. Therefore in order to minimize oxidative damage, it is further broken down 
to molecular Oxygen by the activities of the enzymes Catalase and Glutathione Peroxidases (Finkel and Holbrook, 2000).

Oxidative stress is defined as any condition where there is a net increase in the levels of ROS either due to overproduction of ROS leading to overwhelming the ROS metabolizing enzymes, or the inefficient break down of ROS by these enzymes because of insufficiency, loss of catalytic activity or improper targeting to their correct subcellular locations. The overproduction of ROS can be due to a temporary defect in the ETS which can be amplified by a vicious cycle. A small amount of ROS may be initially generated which inhibits the activity of $\alpha$-Ketoglutarate dehydrogenase (Chinopoulos et al., 2002) and Aconitase (Verniquet et al., 1991), important enzymes of the Kreb's cycle occurring in the mitochondria. This inhibits the production of $\mathrm{NAD}(\mathrm{P}) \mathrm{H} 2$ which is the actual source of electrons to the ETS (Chinopoulos et al., 2002). Normally complexes I, III and IV couple the flow of electrons from a specific donor to a specific acceptor, to the pumping of protons against their concentration gradient. This causes an electrochemical gradient across the inner membrane of the mitochondria such that the outside (Inner luminal space) is positively charged and the inside is negatively charged. This is designated the mitochondrial membrane potential $\left(\psi_{\mathrm{m}}\right)$ (Chen, 1988). When protons flow down their electrochemical gradient through a multisubunit complex called the $\mathrm{F}_{0} \mathrm{~F}_{1}$ ATPase, the free energy released upon undoing the gradient, is coupled to the synthesis of ATP from ADP and $\mathrm{P}_{\mathrm{i}}$ by a process called rotational catalysis (Hatefi, 1985). In the event of depletion of $\mathrm{NAD}(\mathrm{P}) \mathrm{H} 2$, the proper functioning of Complexes I, III and IV are compromised, thus inhibiting the pumping of protons and establishment of the membrane potential. Because of dissipation of the proton gradient, protons are no 
longer available to flow through the $\mathrm{F}_{0} \mathrm{~F}_{1}$ ATPase complex and as a result the production of ATP is halted (Maechler et al., 1999). Interestingly when such a scenario occurs, the $\mathrm{F}_{0} \mathrm{~F}_{1}$ ATPase complex can actually rotate in the opposite direction and hydrolyze ATP, thus dropping ATP levels further, while pumping protons in the opposite direction to maintain the mitochondrial membrane potential (Yasuda et al., 2001). This process becomes highly dependent on glycolysis (Budd and Nicholls, 1996; Leyssens et al., 1996; Scott and Nicholls, 1980). Since ROS is generated mostly at complexes I and III of the ETS (Finkel and Holbrook, 2000), this shift to a glycolytic mode may serve to reduce the production of ROS although this is highly debatable (Mailloux and Harper). However studies have shown that $\mathrm{H}_{2} \mathrm{O}_{2}$ inhibits the activity of a $\mathrm{Na}^{+} / \mathrm{Ca}^{2+}$ antiporter on the surface of the mitochondrial inner membrane, leading to a rise of matrix $\mathrm{Ca}^{2+}$ (Jornot et al., 1999) (Maechler et al., 1999). This leads to the opening of non-selective pores or channels on the surface of the mitochondria, a phenomenon termed Mitochondrial Permeability Transition (MPT) (Szabo and Zoratti, 1991; Szabo and Zoratti, 1992). Due to the loss of a functional barrier between the matrix and the inner membrane of the mitochondria, further dissipation of the electrochemical gradient ensues (Lemasters et al., 1998). Interestingly ROS that have leaked out of one mitochondrion can diffuse to an adjacent one and induce MPT. The resulting loss of the proton gradient further generates ROS and the chain reaction is carried forward, a phenomenon termed ROS Induced ROS Release (RIRR) (Zorov et al., 2000; Zorov et al., 2006). Thus RIRR can function to produce a gross over-amplification of oxidative stress in a relatively short period of time. 
Oxidative stress can also occur as a consequence of impaired activity of the ROS metabolizing enzymes (Mates et al., 1999). This can be due to defects in synthesis of the enzyme or mutations that inactivate it. Catalase deficiency has been implicated in various diseases like Diabetes Mellitus and Hypertension (Laszlo et al., 2004). Hungarian Acatalasemia is a disease caused by a mutation in the Catalase gene (Goth et al., 2000). Mutant SOD has also been implicated in familial Amyotropic Lateral Sclerosis (Orrell et al., 1995). Inhibition of Catalase activity by 3-Amino Triazole leads to a net increase in cellular ROS levels, causes oxidative damage to proteins and DNA, depolarizes mitochondria and reduces cell proliferation, basically signs of a premature aging phenotype (Koepke et al., 2008). The import of Catalase into peroxisomes has been shown to progressively deteriorate with cellular passage number in culture. This is accompanied by an increase in ROS levels which further prevents the import process (Legakis et al., 2002). Restoration of Catalase import into peroxisomes in late passage cells by using a more potent peroxisomal targeting signal, reduces cellular ROS levels, restores mitochondrial inner membrane potential, as well as delays senescence in culture (Koepke et al., 2007). Since accelerated replicative senescence is one of the hallmarks of HGPS cells in culture (Allsopp et al., 1992; Huang et al., 2008), similar possible effects of increased oxidative stress, either by inactivation of Catalase or by defective peroxisomal import on the proliferative capacity of cells, underscores the importance of studying oxidative stress in the context of premature aging syndromes. Also since Catalase targeted to the mitochondria has successfully extended lifespan in mice along with the amelioration of age related cardiac 
malformations (Dai et al., 2009; Schriner et al., 2005; Treuting et al., 2008), a similar manipulation in mouse models of HGPS may be worthwhile.

\section{Oxidative stress and the nuclear lamina: the role of prelamin A.}

For a long time, HIV aspartyl protease inhibitors that were used to treat HIV patients led to off target effects like cardiovascular complications, lipodystrophy, tissue necrosis and other pro-inflammatory responses. This was found to be a result of elevated oxidative stress (Chai et al., 2005; Vincent et al., 2004). As a result a series of studies ensued where a variety of HIV protease inhibitors were tested on human adipocytes, macrophages, macrophage derived foam cells and human umbilical vein endothelial cells and they significantly increased oxidative stress (Jiang et al., 2007; Lagathu et al., 2007; Wang et al., 2007). However the conceptual linkage between perturbation of the nuclear lamina and oxidative stress was established by the finding that Lopinavir (LPV), one of the HIV protease inhibitors inhibits the Zmpste24 protease that is involved in the processing of Lamin A (Coffinier et al., 2007). This sparked an interest in the causal relationships between Lamin A perturbation and oxidative stress and in two independent studies, the authors demonstrated that the HIV protease inhibitors Lopinavir and Indinavir (IDV) reduce cell viability, induce senescence, depolarize Mitochondria and increase ROS levels and all of this is attributed to the membrane accumulation of prelamin A (Caron et al., 2007; Lefavre et al., 2010). However the finding that is most striking in both these studies is the formation of dysmorphic nuclei, a feature that is shared with HGPS. Also quite noteworthy is the fact 
that all the cellular defects outlined above were also produced by LMNA mutations that are causal to various Lipodystrophies (Caron et al., 2007). The $\Delta 50$ mutant of Lamin A that causes HGPS is likely to behave similar to prelamin A owing to the retention of the farnesyl anchor (De Sandre-Giovannoli et al., 2003; Eriksson et al., 2003b). Since LPV treatment of normal human fibroblasts accumulates prelamin A, disrupts the Ran gradient and reduces nuclear SUMOylation (Kelley et al., 2011), the striking resemblance between the cellular effects of prelamin A and Progerin encourages the use of prelamin $\mathrm{A}$ as a progerin mimetic. Considering the ability of prelamin $\mathrm{A}$ induction to produce ROS, the use of HIV protease inhibitors to address the growing concept of oxidative stress in HGPS and whether the cellular defects identified in HGPS are related to the ROS induction, appears to be an attractive approach.

\section{Oxidative stress and the Ran system}

In addition to causing reduced cell viability and senescence as outlined above, oxidative stress affects the Ran system, thereby inhibiting nuclear import of proteins. Oxidative stress was shown to inhibit nuclear import in yeast and also cause a disruption of the Gsp1p gradient, the yeast homologue of Ran (Stochaj et al., 2000). In a permeabilized cell assay for reconstituted nuclear import (Adam et al., 1990), incubation of the cytosolic cocktail with $\mathrm{H}_{2} \mathrm{O}_{2}$ inhibited the nuclear import of the cargo and this was reversible by Catalase treatment. However a similar treatment of the isolated nuclei themselves did not affect import (Czubryt et al., 2000). Interestingly superoxide generating systems when added to the isolated nuclei did affect import of 
the cargo while the same treatment of the cytosolic import cocktail did not. This implies that $\mathrm{H}_{2} \mathrm{O}_{2}$ and Superoxides have different mechanisms for inhibiting import and they work on cytosolic and nuclear components respectively (Czubryt et al., 2000). In the same study, the authors also reported a disruption of the Ran protein gradient in intact cells (Czubryt et al., 2000).

The disruption of the Ran gradient by $\mathrm{H}_{2} \mathrm{O}_{2}$ was confirmed by another group where they also reported a concomitant mislocalization of Importin a from the cytosol to the nucleus and an inhibition of nuclear import in intact cells in a time dependent manner (Miyamoto et al., 2004b). In a follow up paper, the same group demonstrated that the disruption of the Ran gradient in response to $\mathrm{H}_{2} \mathrm{O}_{2}$ treatment is a result of ATP depletion (Yasuda et al., 2006). Since the synthesis of adenine and guanine nucleotides are closely coupled to each other by interconversion, a drop in ATP levels is expected to reduce the cellular GTP levels and thereby also affect RanGTP production. Indeed the levels of RanGTP were much lower in cells treated with $\mathrm{H}_{2} \mathrm{O}_{2}$ (Yasuda et al., 2006). Another study showed that ATP depleting agents drastically inhibit nuclear import and this is due to a decrease in free GTP levels that causes a drop in total RanGTP (Schwoebel et al., 2002). Further in-depth analysis of the mechanism of inhibition of nuclear import by oxidative stress revealed defects in multiple components of the nuclear transport machinery (Kodiha et al., 2004). Nup153, a nucleoporin on the nuclear side of the NPC is mislocalized and so is Importin $\beta$. The docking of Importin $\alpha / \beta$ cargo complexes on the NPC is reduced. Ran, Importin $\beta$ and Nup153 are degraded in response to oxidative stress (Kodiha et al., 2004). Since the Ran protein gradient is disrupted in HGPS cells (Kelley et al., 2011), a possible role of oxidative stress may be 
warranted, especially since oxidative stress is a newly emerging concept in the field of premature aging syndromes and is likely to play a central role in disease development and progression.

\section{Oxidative stress and the SUMOylation machinery}

The link between oxidative stress and the SUMOylation machinery came from independent findings that global changes in SUMOylation occur upon treating cells with $\mathrm{H}_{2} \mathrm{O}_{2}$ (Manza et al., 2004; Saitoh and Hinchey, 2000), and hypoxic stimulation in adult mice causes an increase in SUMO1 expression (Shao et al., 2004). This was followed by studies where cerebral ischemia in adult mice led to a massive increase in conjugation by both SUMO1 and SUMO2/3 (Cimarosti et al., 2008; Yang et al., 2007a, 2008). This dynamic behavior of SUMO conjugation in response to oxidative stress triggered investigations at the mechanistic level. The first molecular perspective was provided by the Melchior group where they demonstrated that low concentrations of $\mathrm{H}_{2} \mathrm{O}_{2}$ decrease SUMO conjugation while high concentrations (Higher than $100 \mathrm{mM}$ ) increase it (Bossis and Melchior, 2006). They also showed that low concentrations of $\mathrm{H}_{2} \mathrm{O}_{2}$ cause a reversible disulfide formation between $\mathrm{Ubc} 9$ and $\mathrm{Uba} 2$ between their catalytic cysteines. The resulting loss of catalytic activity of both Ubc9 and Uba2 leads to the decrease in SUMO conjugation. However at high concentrations, the SENPs are also inhibited and this overrides the effect of inhibition of $\mathrm{Ubc9}$, thereby leading to a net increase in SUMO conjugation (Bossis and Melchior, 2006). This fostered the idea that enzymes of the SUMOylation machinery could act as redox sensors and that the 
catalytic inactivation of Ubc9 could serve to be an early event in the response of cells to oxidative stress (Bossis and Melchior, 2006). This could be particularly important in the context of HGPS because nuclear SUMOylation is drastically reduced in HGPS cells (Kelley et al., 2011) and therefore it is worthwhile to study if components of the SUMOylation machinery are affected in HGPS and if this is a result of increased oxidative stress.

With these goals in mind, we set out to determine if there was a link between the reduction of nuclear SUMOylation and loss of the Ran gradient in HGPS. We discovered that the mis-localization of Ubc9 from the nucleus to the cytoplasm in HGPS cells is causal to the loss of the Ran gradient. We also established that Ubc9 depends on the Ran gradient for its nuclear import, thus demonstrating a feed-back mode of regulation. The loss of the Ran gradient induces oxidative stress and this in principle can further worsen the Ran and Ubc9 defects. Our results suggest a complex, mutually regulatory signaling pathway involving the redox machinery, the Ran system and the SUMOylation machinery. This pathway when disrupted, could contribute to changes that promote cellular, tissue and clinical phenotypes in HGPS. 


\section{Chapter 2. THE DEFECTIVE SUMOYLATION MACHINERY IN HGPS CAUSES A DISRUPTION OF THE RAN PROTEIN GRADIENT.}

\section{SUMMARY}

Disruption of the Ran protein gradient and loss of nuclear SUMOylation was recently reported in fibroblasts from HGPS patients. The strong degree of correlation between the defects led us to propose that these pathways could be related. Disruption of nuclear SUMOylation disrupted the Ran gradient, indicating that the Ran gradient defects are downstream. Probing into the mechanism of reduced nuclear SUMOylation in HGPS, we discovered defects in the nuclear localization of Ubc9, the SUMO E2. Restoring the nuclear localization of Ubc9 in the face of Progerin rescued the Ran gradient, thus confirming the hypothesis that nuclear SUMOylation regulates the Ran gradient. This enabled the restoration of the nuclear import of a Ran dependent cargo, namely the nucleoporin TPR and an indirect downstream target of SUMOylation, namely H3K9 trimethylation. Thus in the context of HGPS, Ubc9 induced nuclear SUMOylation has important roles to play and defects in its import may have a key role in the progression of HGPS at the cellular level.

Note: All data in this chapter have been generated by Sutirtha Datta and were published as part of a larger study (Kelley et al., 2011). 


\section{INTRODUCTION}

The Hutchinson-Gilford Progeria Syndrome (HGPS) is a premature aging syndrome in children, which is caused by a mutation in the Lamin A gene (De SandreGiovannoli et al., 2003; Eriksson et al., 2003a). Recently we have characterized some of the cellular defects in HGPS including the loss of the Ran protein gradient and a reduction of nuclear SUMOylation (Kelley et al., 2011). An intact Ran gradient is necessary for nuclear import and export and is maintained by the activity of RCC1 which is a chromatin bound enzyme that exchanges RanGDP with RanGTP, a cytosolic Nuclear Pore Complex associated protein RanGAP that catalyzes the hydrolysis of RanGTP to Ran GDP and NTF2, a shuttling protein that carries RanGDP back into the nucleus after an export cycle (Bischoff and Ponstingl, 1991; Bischoff et al., 1994b; Nemergut et al., 2001; Pemberton and Paschal, 2005; Ribbeck et al., 1998; Smith et al., 1998a). Biochemical analysis of RanGAP revealed it to be SUMOylated (Mahajan et al., 1997; Matunis et al., 1996). SUMO is a post translational modification of proteins that belongs to the Ubiquitin superfamily (Mahajan et al., 1997; Matunis et al., 1996). After processing of the peptide by SUMO proteases or SENPS, it goes through a catalytic cycle involving an activator E1 (Heterodimer of AOS1 and Uba2) and a SUMO E2 ligase Ubc9, which with the assistance of SUMO E3s, specifically modify Lysines on the target protein (Geiss-Friedlander and Melchior, 2007). SUMO modified proteins can be deSUMOylated by the activity of the SENPs. This dynamic modification regulates various processes in the cell ranging from protein interactions to trafficking and activity (Geiss-Friedlander and Melchior, 2007). 
There are four paralogs of SUMO in mammals of which three are ubiquitously expressed. These are SUMO1, SUMO2 and SUMO3. Of these SUMO2 and 3 are 97\% identical and are hence grouped under a single category SUMO2/3 (GeissFriedlander and Melchior, 2007). Interestingly only SUMO2/3 has been reported to be reduced in HGPS and not SUMO1 (Kelley et al., 2011). The SUMO E2 Ubc9 is the sole E2 in cells and hence does not discriminate between the SUMO paralogs (GeissFriedlander and Melchior, 2007). It is predominantly nuclear although cytosolic and NPC associated distribution has also been reported (Pichler et al., 2002; Zhang et al., 2002). At the NPC, Ubc9 forms a complex with Nup358, a cytosolic Nucleoporin and SUMO1 modified RanGAP. By immunogold electron microscopy, Ubc9 has also been detected on the nuclear side of the NPC (Zhang et al., 2002). Its nuclear import is carried out by Importin 13, an import receptor of the Importin $\beta$ superfamily (Mingot et al., 2001a). Although the genetic basis of HGPS has been documented, very little is known about the cellular mechanisms of progression of the disease. Our previous findings on Ran and SUMO 2/3 defects led us to ask if these defects were part of the same or different pathways. In the process, we demonstrated that nuclear SUMOylation works upstream of the Ran gradient and the SUMO E2 Ubc9 is mislocalized in HGPS. Restoration of the nuclear localization of Ubc9 in the presence of Progerin rescued nuclear SUMO2/3 levels, the Ran gradient, $\mathrm{H} 3 \mathrm{~K} 9$ trimethylation levels in the nucleus and corrected the defective import of nucleoporin TPR. Thus we show that the SUMOylation machinery has a central role in HGPS and may be one of the key mediators of the disease phenotypes. 


\section{MATERIALS AND METHODS}

\section{Cell culture}

Primary human fibroblasts from HGPS patients (AGO1972, AG11498, and AGO3199) and a clinically normal father (AGO8469) of an HGPS patient were obtained from the Coriell Cell Repository (Camden, NJ). These are designated HGPS 1972, HGPS 1498, HGPS 3199 and Normal 8469 respectively. Primary fibroblasts were grown at $37^{\circ} \mathrm{C}$ in $5 \% \mathrm{CO}_{2}$ in minimal essential medium (MEM) (Gibco) containing $15 \%$ FBS (Hyclone), $1 \%$ MEM vitamin solution (HyClone), 1\% penicillin/streptomycin (Gibco) and $1 \mathrm{mM}$ sodium pyruvate (Gibco). The passage number for cells in various experiments was between 8 and 25 . HeLa cells were grown at $37^{\circ} \mathrm{C}$ in $5 \% \mathrm{CO}_{2}$ in Dulbecco's modified Eagle's medium (DMEM) (Gibco/lnvitrogen) containing 5\% newborn calf serum (Gibco/Invitrogen), 5\% fetal bovine serum (Gibco/Invitrogen) or Atlanta Biologicals) and $1 \mathrm{mM}$ sodium pyruvate (Gibco/Invitrogen).

\section{Plasmids and cloning.}

pCDNA3-HA-Lamin, pCDNA3-HA-Progerin and mCherry-SUMO 2 constructs were generated as outlined in (Kelley et al., 2011). pCMV5-FLAG-Ubc9, pCMV5-FLAGUbc9-C93S and pCMV5-SENP2-catalytic domain (aa 317 to 590) were generously provided by David Wotton. Transport signal fusions to Ubc9 were generated by using the nuclear localization signal (NLS) from the simian virus 40 (SV40) large T antigen and the nuclear export signal (NES) from the protein kinase inhibitor (PKI). FLAG-NLSUbc9 was made by amplifying Ubc9 from pCMV5-FLAG-Ubc9 to introduce BamHI and Xhol sites and was then cloned into a pcDNA-FLAG-NLS backbone. FLAG-NES-Ubc9 
was generated by a similar strategy.

\section{Transfection}

The cells to be transfected were plated and grown in 6 well plates to a density of about $60 \%$ for 24 hours, followed by transfection with Transfectin (Biorad) according to the manufacturer's instructions. Briefly the transfectin reagent was mixed with a total of $1 \mu \mathrm{g}$ of plasmid DNA at a ratio of $1: 1$ in $100 \mu$ l of serum free DMEM (Gibco/Invitrogen) for each well of a 6 well plate. After an incubation period of 20 minutes, the mixture was added to the cells and processed for immunofluorescence microscopy or immunoblotting after 24 hours.

\section{Immunoblotting}

Cells were transfected for 24 hours followed by cell lysis using standard lysis buffer. Extracts were sonicated prior to loading on SDS-polyacrylamide gels. SDSPAGE and immunoblotting were performed by standard methods using primary antibodies anti-HA (MAb 16B12, Santa Cruz), anti-Ubc9 (pAb, catalog number ab33044; Abcam) and Tubulin (MAb 1-A2 Sigma), peroxidase-labeled secondary antibodies, followed by detection by chemiluminescence using ECL reagent.

\section{Immunofluorescence microscopy}

Cells were grown on glass coverslips, fixed with $3.7 \%$ formaldehyde for $20 \mathrm{~min}$, and permeabilized in $0.2 \%$ Triton X-100 for 5 min. Coverslips were incubated in primary antibody diluted in IF microscopy blocking buffer (1X phosphate-buffered saline [PBS], $2 \%$ bovine serum albumin, $2 \%$ newborn calf serum) for at least 1 hour and overnight in 
the case of Ubc9. Antibodies to the following proteins were used for IF: Ran (monoclonal antibody[Mab], catalog number 610341; BD Biosciences), Lamin A (polyclonal antibody [pAb], catalog number PRB-113c; Covance), H3K9me3 (pAb, catalog number ab8898; Abcam), TPR (pAb kindly provided by Larry Gerace), Ubc9 (pAb, catalog number ab33044; Abcam), SUMO2/3 (pAb SUMO-2 [Zymed] and MAb 8A2 anti-FLAG epitope (MAb M2; Sigma), OctA anti-FLAG (Santa Cruz), RanGAP (MAb 21c7; Zymed), and anti-HA (MAb 16B12 and pAb; both from Santa Cruz). The secondary antibodies for IF microscopy were diluted in blocking buffer and incubated for $1 \mathrm{~h}$. The antibodies used were fluorescein isothiocyanate (FITC)-labeled donkey antimouse, Cy3-labeled donkey anti-mouse, FITC-labeled donkey anti-rabbit, and Cy3labeled donkey anti-rabbit antibodies (all from Jackson Immunoresearch). Wide-field microscopy was performed with a Nikon Eclipse E800 upright microscope using a 40X 1.0 NA oil immersion objective and recorded with a Hamamatsu C4742-95 chargecoupled device camera using OpenLab software (Perkin Elmer). Confocal imaging was done on Zeiss LSM 510 and Zeiss LSM 510-META microscopes with $40 \mathrm{X}$ and 100X 1.3 NA oil immersion objectives using Axiovision software (Carl Zeiss).

\section{Image quantification and statistical analyses.}

Quantitative analysis of IF microscopy images (ratios of the nuclear concentration to the cytoplasmic concentration [N/C ratios]) was performed by using ImageJ image processing software $(\mathrm{NIH})$. More than 50 cells each from at least three experiments were quantified by circling relevant areas namely the nucleus and the cytoplasm and measuring the mean fluorescent intensities. Quantification of mCherry-SUMO2 levels was performed by using ImageJ, by outlining individual cells and corresponding nuclei 
and deriving the ratio of total nuclear SUMO fluorescence intensity to the total cellular SUMO fluorescence. Data were binned using Excel (Microsoft) prior to plotting as histograms, with bin sizes stated in the figure legends. The " $t$ test: two sample assuming equal variances" function of Excel (Microsoft) was used to calculate $p$ values, and the type 2 two tailed $p$ value was used. 


\section{RESULTS}

\section{Progerin reduces nuclear SUMO2/3 levels.}

Since endogenous SUMO2/3 levels were reduced in HGPS cells as well as HeLa cells transfected with HA-Progerin (The mutant form of Lamin A expressed in HGPS), we looked for additional approaches to support our initial observations. We made use of a fluorescent fusion protein (mcherry-SUMO2/3) and cotransfected it with HA Lamin and Progerin followed by triple label immunofluorescence microscopy to detect WT and mutant Lamin A, Ran and SUMO. While the HA-Lamin transfected cells had normal mcherry-SUMO2/3 distribution and an intact Ran gradient, a subset of cells transfected with HA-Progerin had a disrupted Ran gradient and in the same cells, mcherry-SUMO 2/3 was mislocalized (Fig $1 \mathrm{~A}$ ). The ratio of the nuclear to the total cellular intensity of mcherry-SUMO2/3 was plotted as a histogram, which reveals a quantitative reduction of SUMO2/3 with Progerin but not with WT Lamin A (Fig 1 B). This indicates that nuclear SUMO2/3 levels are reduced in HGPS and this may involve a combination of reduction of SUMOylation in the nucleus and a defective nuclear import of SUMO modified proteins.

\section{Inhibition of nuclear SUMOylation disrupts the Ran protein gradient.}

The correlation between SUMO2/3 levels and the Ran protein gradient in HGPS cells led us to explore whether nuclear SUMOylation and the Ran protein gradient are linked and whether the disruption of one affects the other. To that effect, we transfected 
Figure 1. Progerin reduces nuclear SUMO2/3 levels. (A) Images from HeLa cells triple labeled for HA-progerin and HA-WT lamin A (purple), endogenous Ran (green), and mCherry-SUMO2 (red). (B) Histograms (bin size $=0.1$ ) of Cherry-SUMO2 levels (nuclear/total) in progerin and lamin A transfected cells. "Copyright @ American Society for Microbiology, [Mol Cell Biol. 2011 August; 31(16): 3378-3395]" 
Figure 1
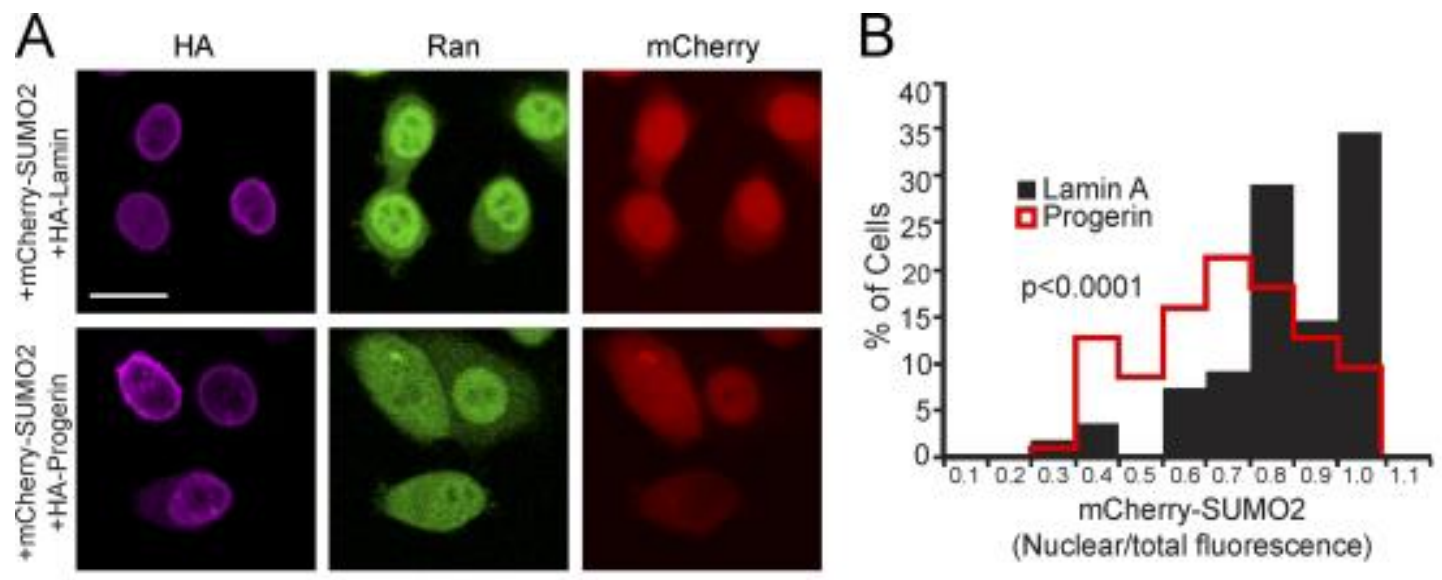
HeLa cells with a catalytic mutant of Ubc9 (C93S mutant) that reduces SUMOylation by acting as a dominant negative protein (Chakrabarti and Nucifora, 1999) ,or the catalytic domain (CD) of the SUMO protease SENP2, which cleaves SUMO from target proteins (Mukhopadhyay and Dasso, 2007). FLAG-tagged WT and C93S forms of Ubc9 and the FLAG-tagged SENP CD were introduced into HeLa cells and examined by double-label IF microscopy for FLAG and Ran or SUMO2/3 and Ran. The expression of FLAG-Ubc9 C93S and the FLAG-SENP CD was sufficient to reduce nuclear levels of Ran (Fig 2A). Cells with reduced levels of nuclear SUMO2/3 signal also showed a loss of the Ran protein gradient (Fig 2A). Thus, a reduction of the level of nuclear SUMOylation is sufficient to disrupt the Ran gradient hinting towards the possibility that the same mechanism may be at play in HGPS.

RanGAP is one of the key regulators of the Ran system, and the SUMOylation of RanGAP regulates its anchorage to the NPC (Mahajan et al., 1997; Matunis et al., 1996). In order to ask whether the observed Ran gradient defects were a result of defective targeting of RanGAP in response to decreased SUMOylation, we examined RanGAP distribution with progerin and SENP CD expression, conditions where SUMOylation is markedly reduced. In cells where the SENP CD and progerin reduced nuclear SUMOylation and disrupted the Ran protein gradient, RanGAP was still targeted to the NPC (Fig. $2 \mathrm{~B}$ and C). This result together with the fact that RanGAP is localized to the NPC in HGPS patient cells (Kelley and Paschal, unpublished observations) indicate that the Ran protein gradient disruption in HGPS is not caused by a failure of RanGAP targeting to the NPC. 
Figure 2. Inhibition of SUMOylation disrupts the Ran protein gradient. (A) HeLa cells were transfected with FLAG-tagged forms of WT Ubc9, a catalytic mutant of Ubc9 (C93S) that acts as a DN protein, and the SENP CD. Double-label IF microscopy for FLAG (red) and endogenous Ran (green) showed that the Ran protein gradient is disrupted by the expression of Ubc9 C93S and the SENP CD (left) under conditions where these factors reduce nuclear SUMO2/3 levels (right). (B and C) The SENP CD (B) and HA-progerin (C) do not disrupt endogenous RanGAP targeting to the NPC. Scale bars, $20 \mu \mathrm{m}$. "Copyright $\odot$ American Society for Microbiology, [Mol Cell Biol. 2011 August; 31(16): 3378-3395]" 
Figure 2

A
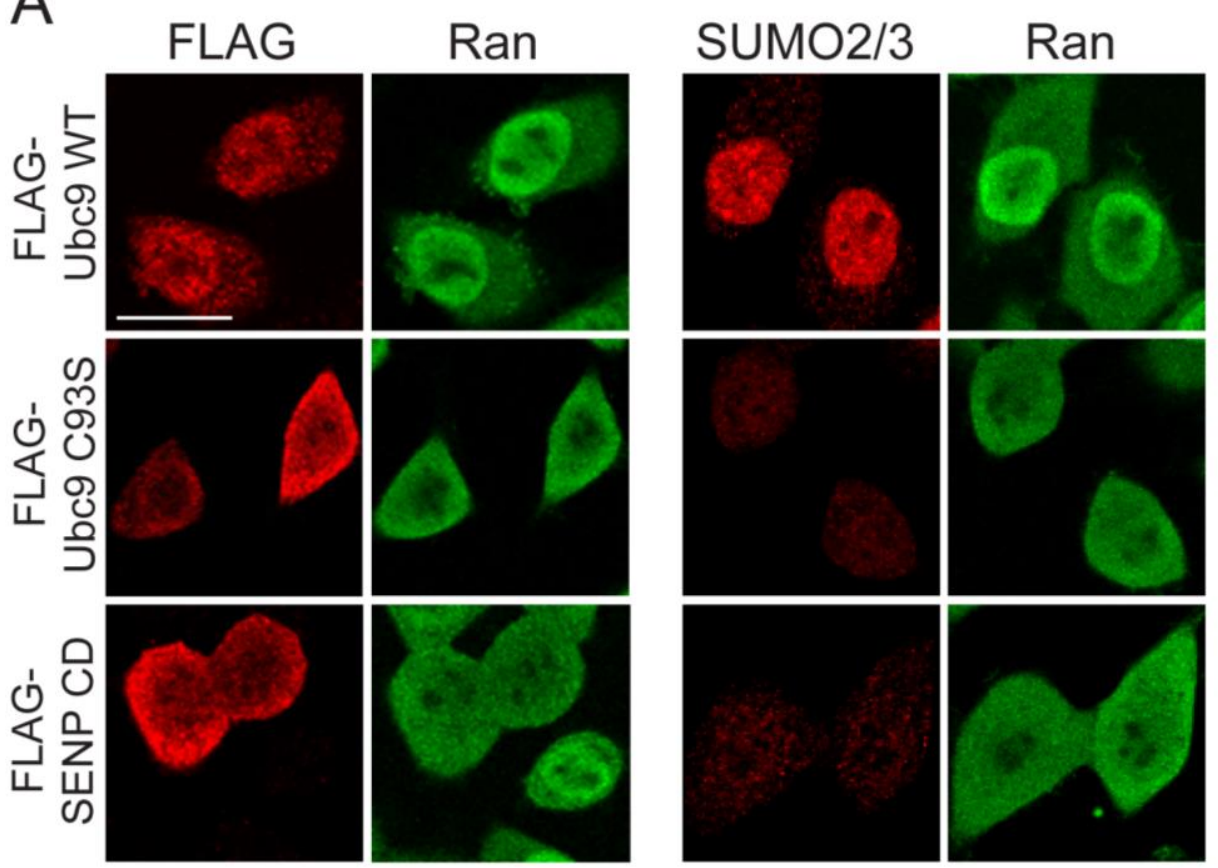

B

FLAG RanGAP
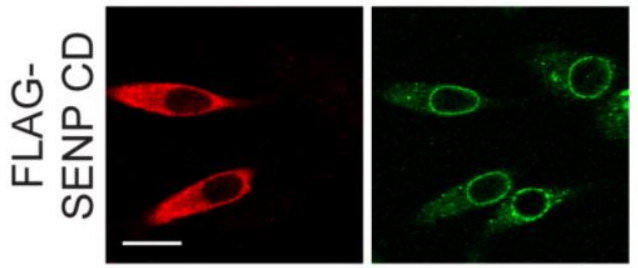

FLAG SUMO2/3

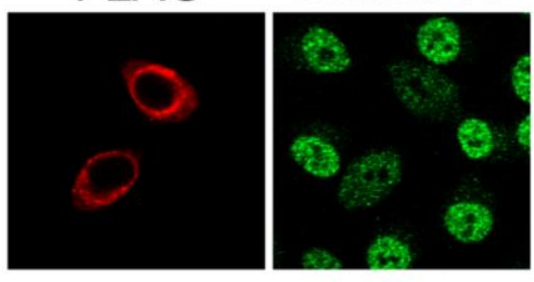

C
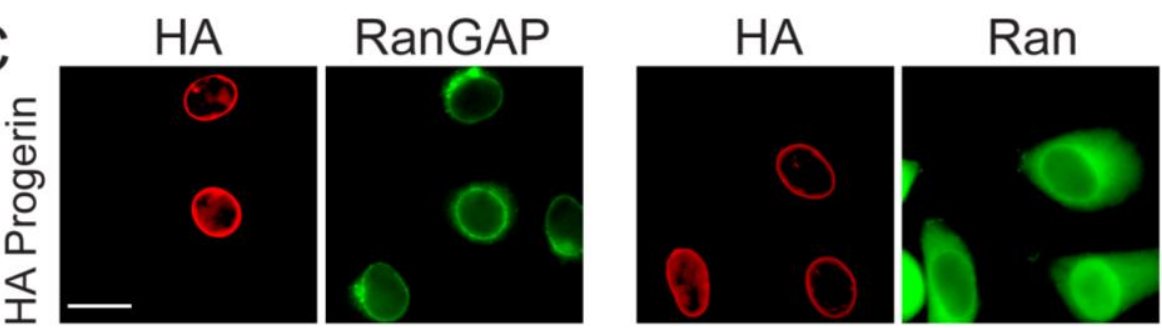


\section{Defective nuclear localization of Ubc9 in Progeria}

The reduced nuclear levels of SUMO2/3 caused by progerin expression (Fig 1) led us to hypothesize that progerin could inhibit the activity or localization of a component that is critical for SUMOylation. The sole E2 for SUMO conjugation is Ubc9, an enzyme found in both the nucleus and the cytoplasm (Lee et al., 1998; Zhang et al., 2002). In normal human fibroblasts, Ubc9 was mostly nuclear, with a small pool detected in the cytoplasm (Fig 3 A). In progeria fibroblasts, however, Ubc9 was localized predominantly to the cytoplasm. Moreover, in cells where the distribution of Ubc9 was predominantly cytoplasmic (HGPS 3199), there was a strong disruption of the Ran gradient (data not shown). The reduced nuclear localization of Ubc9 was also recapitulated by progerin expression. HA-progerin but not HA Lamin A transfection was sufficient to cause a quantitative reduction in the N/C distribution of endogenous Ubc9 in HeLa cells with a concomitant reduction of nuclear SUMOylation (Fig 3 B)

\section{Constitutive nuclear localization of Ubc9 restores the Ran gradient in cells expressing progerin.}

The fact that overexpression of factors that reduce nuclear SUMOylation (SENP CD and dominant negative Ubc9) disrupts the Ran gradient (Fig 2), suggests a potential link between SUMOylation and the Ran system, wherein the Ran gradient is regulated by nuclear SUMOylation. Since progerin drives the cytoplasmic localization of Ubc9, we considered whether the loss of Ubc9 from the nucleus is responsible for the disruption of the Ran gradient in HGPS. We engineered Ubc9 with transport signal fusions (SV40 NLS and PKI NES) to force its subcellular localization to the nucleus and cytoplasm 
Figure 3. Reduced nuclear localization of Ubc9 in HGPS. (A) Endogenous Ran (green) and Ubc9 (red) in control (Normal 8469) and progeria (HGPS 3199) fibroblasts. (B) Localization of endogenous Ubc9 (green) and SUMO2/3 (red) in HeLa cells cotransfected with HA-WT lamin A and HA-progerin. Histograms (bin size $=0.25$ ) of Ubc9 N/C ratios in HeLa cells expressing HA-lamin A (black bars) and HA-progerin (red lines). Scale bars, $20 \mu \mathrm{m}$. "Copyright $@$ American Society for Microbiology, [Mol Cell Biol. 2011 August; 31(16): 3378-3395]" 
Figure 3

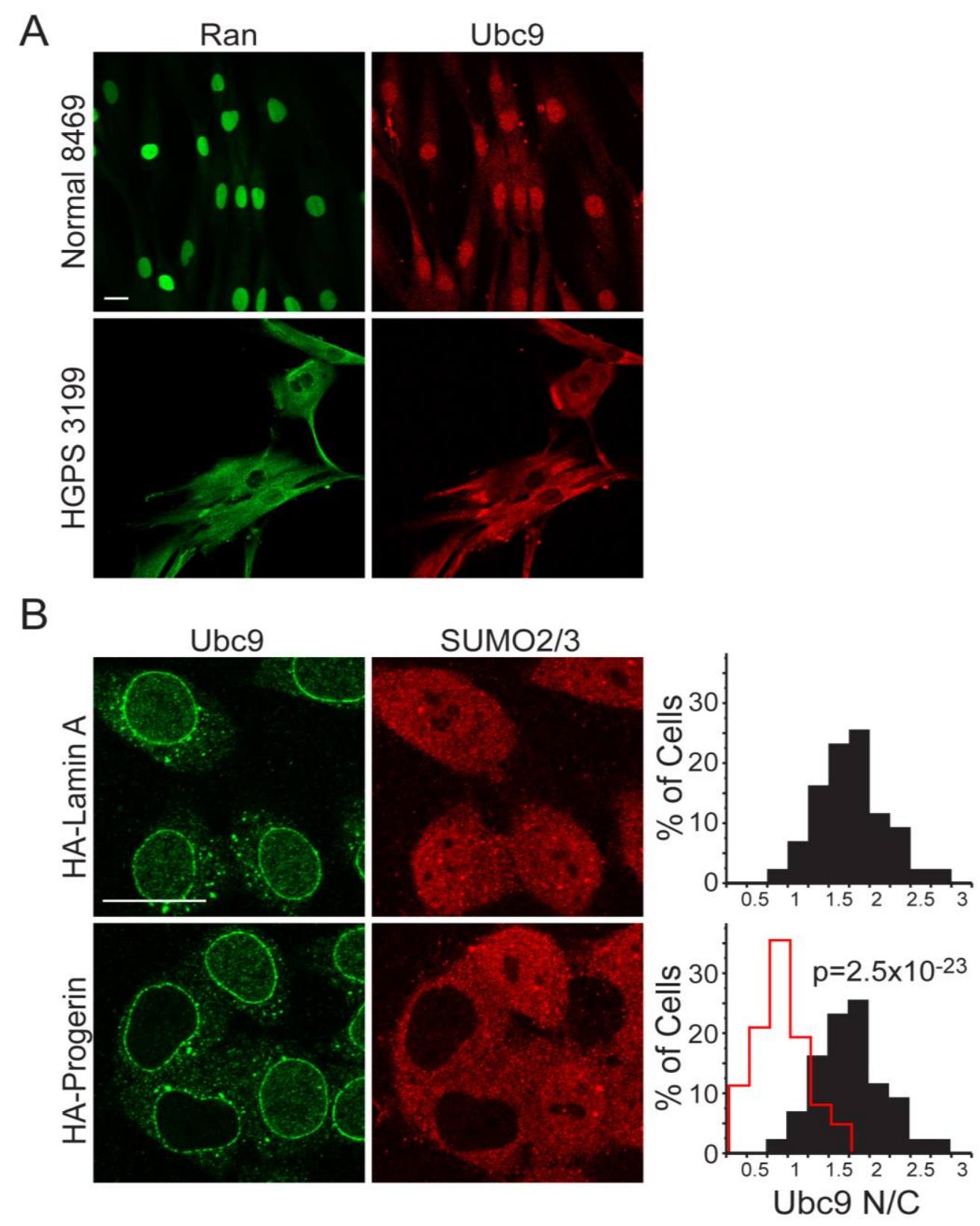


respectively (Fig 4 A); we then tested whether these compartment-specific forms of Ubc9 could rescue the Ran gradient. We also introduced the C93S mutation into the NLS form of Ubc9 to address whether the catalytic function of Ubc9 is necessary for effects on the Ran gradient. Based on the transfection efficiency ( $25 \%)$, expression levels of FLAG-tagged forms of Ubc9 were approximately 2-fold that of endogenous Ubc9 (Fig 4B). As expected, progerin inhibited the nuclear localization of transfected FLAG-tagged WT Ubc9; however, the SV40 NLS fusion rendered both the WT and the catalytically inactive forms of Ubc9 resistant to this effect of progerin (Fig 4 A). The PKI NES fusion with WT Ubc9 was exclusively cytoplasmic in the absence and presence of progerin (Fig $4 \mathrm{~A}$ ).

The four engineered forms of Ubc9 (Fig 4) were each cotransfected with progerin into HeLa cells, and IF microscopy was used to measure Ran N/C levels and the nuclear levels of SUMO2/3. The controls for the experiment were WT Ubc9 cotransfected with WT lamin A (Fig 5, black histograms) and WT Ubc9 cotransfected with progerin (red histograms). Progerin induced a quantitative reduction in the Ran N/C and SUMO2/3 levels (Fig 5, red histograms), both of which were rescued by NLS-Ubc9 expression (green histograms). The catalytically inactive Ubc9 (C93S mutant) (blue histograms) which was targeted to the nucleus and cytoplasmic WT Ubc9 (NES fusion) (plum histograms) targeted to the cytoplasm, both failed to restore the Ran N/C and nuclear SUMO2/3 levels in the presence of progerin (Fig 5). These data show that the SUMO2/3 reduction and Ran gradient disruption by progerin (measured by IF microscopy) can be rescued by forcing Ubc9 into the nucleus and that restoration of the Ran gradient requires the catalytic activity of Ubc9. 
Figure 4. Transport signal fusions that direct nuclear and cytoplasmic targeting of Ubc9. (A) FLAG-tagged SV40 NLS and PKI NES fusions with Ubc9 (green) were expressed in HeLa cells in the absence and presence of HA-progerin (red). Scale bar, $20 \mu \mathrm{m}$. (B) Immunoblotting showing the expression levels of the Ubc9 transport signal fusions relative to those of endogenous Ubc9. "Copyright @ American Society for Microbiology, [Mol Cell Biol. 2011 August; 31(16): 3378-3395]" 
Figure 4

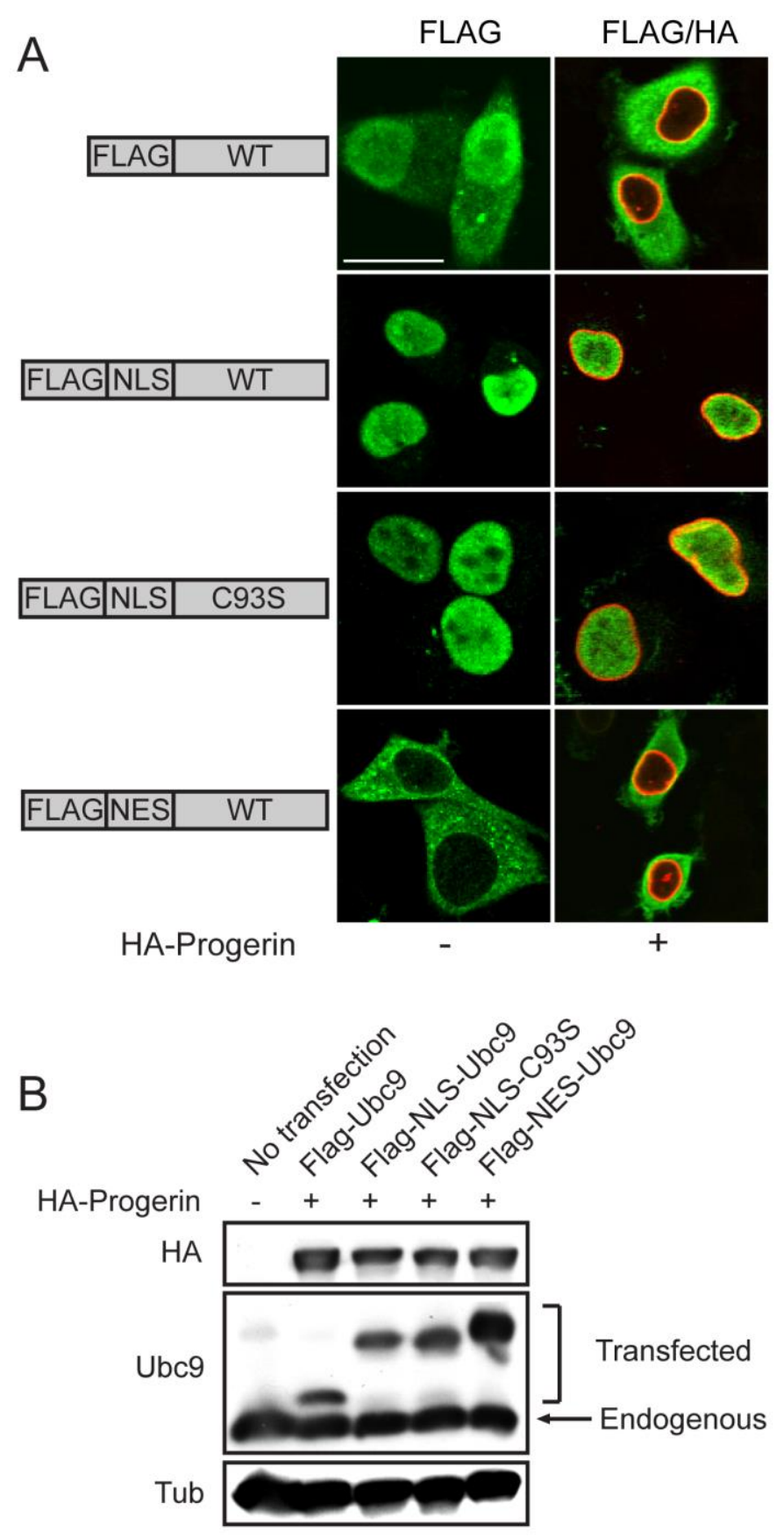


Figure 5. Forcing nuclear localization of Ubc9 rescues the Ran gradient in progerinexpressing cells. HeLa cells were cotransfected with HA-WT lamin A and HA-progerin, together with the transport signal fusions of Ubc9. Histograms show the nuclear levels of SUMO2 (bin size $=0.5$ ) and Ran N/C levels (bin size $=0.25$ ) in HA-positive cells expressing ectopic forms of Ubc9. "Copyright @ American Society for Microbiology, [Mol Cell Biol. 2011 August; 31(16): 3378-3395]" 
Figure 5
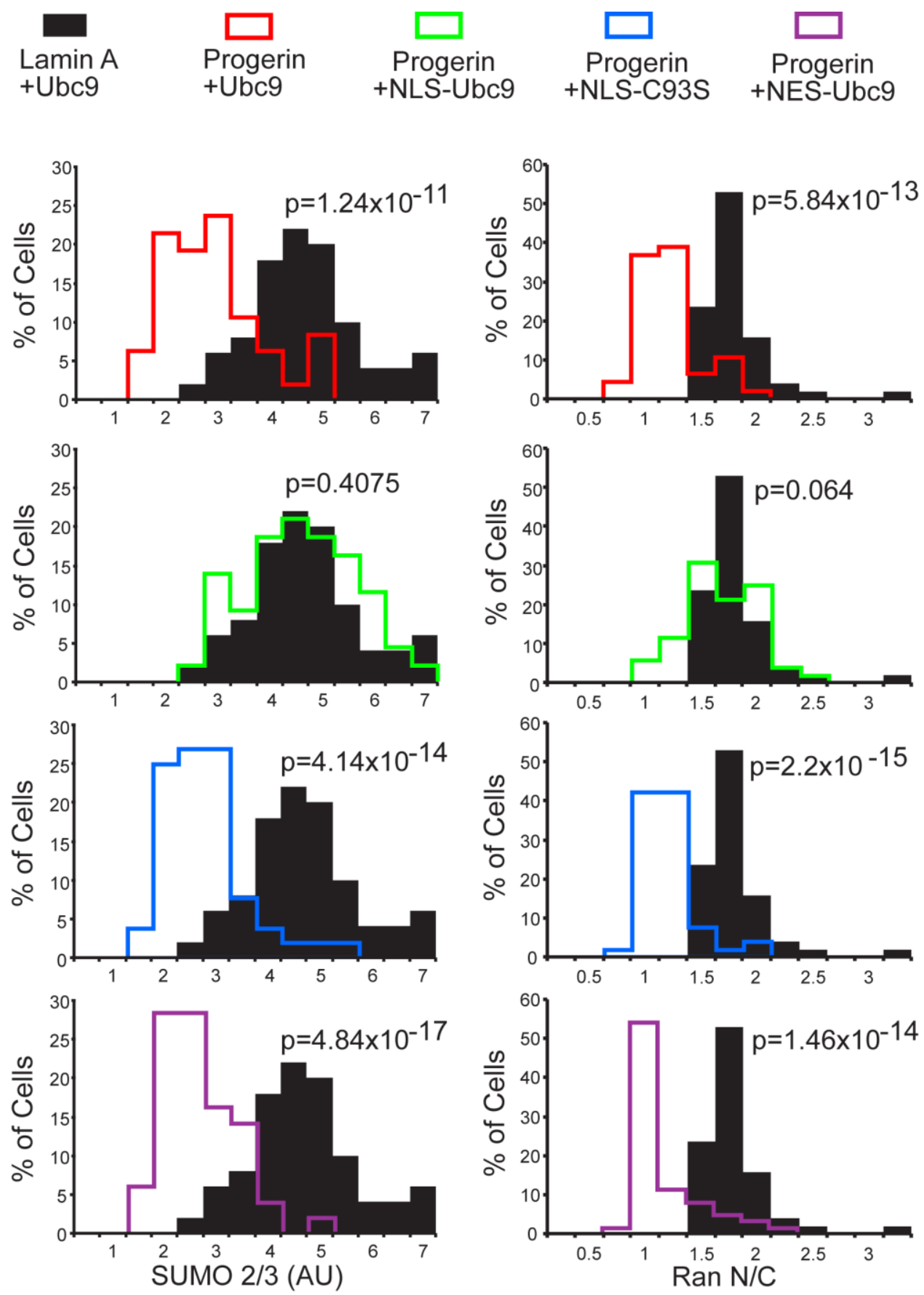


\section{Constitutive nuclear localization of Ubc9 rescues nuclear import of TPR and restores $\mathrm{H} 3 \mathrm{~K} 9$ Trimethylation.}

To determine whether the Ubc9-mediated rescue of the Ran gradient in progerinexpressing cells also rescued Ran-dependent nuclear import, we analyzed the distribution of endogenous TPR in HeLa cells cotransfected with progerin, WT Ubc9, and NLS-Ubc9. TPR is the last nucleoporin that undergoes nuclear import and is assembled on the nuclear side of the NPC to form a basket shaped structure (Bodoor et al., 1999). TPR has been shown to be mis-localized in HGPS cells as well as in HeLa cells with Progerin expression. The reduction in the nuclear import of TPR caused by progerin expression (Fig $6 \mathrm{~A}$, middle) was restored by the cotransfection of NLS-Ubc9 (Fig $6 \mathrm{~A}$, bottom). By measuring the N/C ratios of TPR in cells expressing WT lamin A and WT Ubc9 (Fig 6 B, black histograms), progerin and WT Ubc9 (red histograms), and progerin and NLS-Ubc9 (green histograms), we determined that NLS-Ubc9 expression results in a quantitative increase in TPR import (Fig 6 B). Thus NLS-Ubc9 not only rescues the Ran gradient but also restores nuclear import of certain cargoes whose distribution is affected in HGPS.

One of the cellular hallmarks of HGPS has been shown to be a global alteration in the structure of chromatin, particularly chromatin modification states (Goldman et al., 2004; Scaffidi and Misteli, 2006; Shumaker et al., 2006a). H3K9 Trimethylation has been demonstrated to be reduced in HGPS cells and this reduction co-relates with the degree of disruption of the Ran gradient, suggesting that these pathways might be linked (Kelley et al., 2011). We tested whether the restoration of the Ran gradient via 
Figure 6. Nuclear localization of Ubc9 restores TPR import in cells expressing progerin. (A) IF localization of HA-progerin and endogenous TPR in cells cotransfected with Ubc9. Scale bar, $20 \mu \mathrm{m}$. (B) Histograms showing TPR N/C levels (bin size $=0.5$ ) in HApositive cells expressing engineered forms of Ubc9. "Copyright @ American Society for Microbiology, [Mol Cell Biol. 2011 August; 31(16): 3378-3395]" 
Figure 6

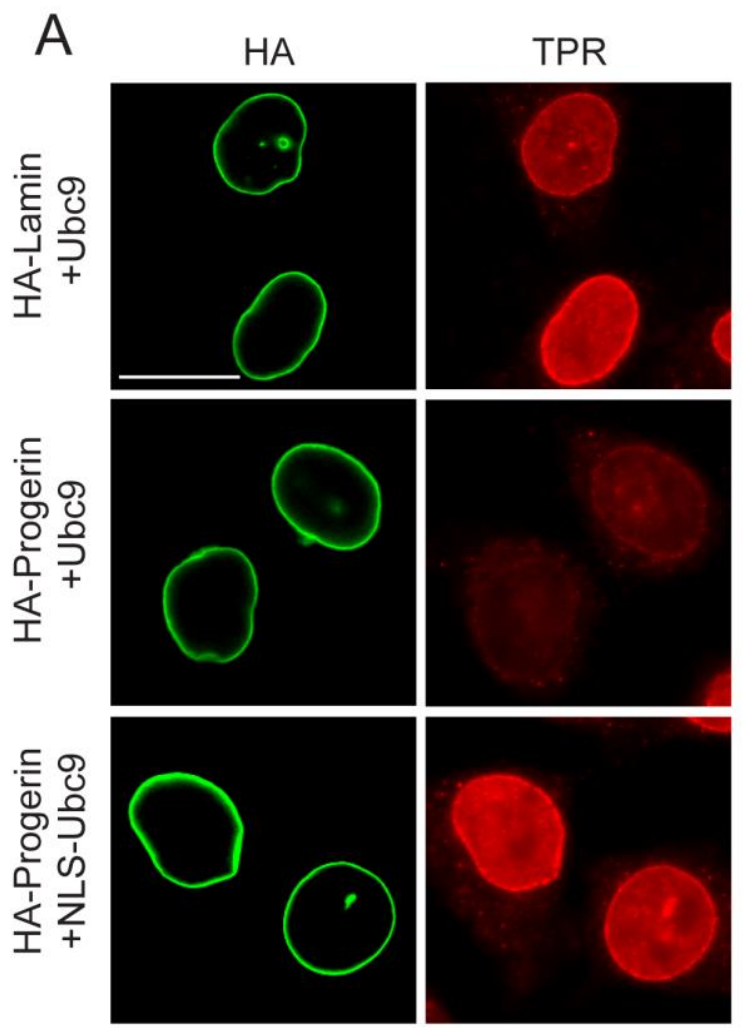

B

Lamin A Progerin Progerin

+Ubc9 +Ubc9 +NLS-Ubc9
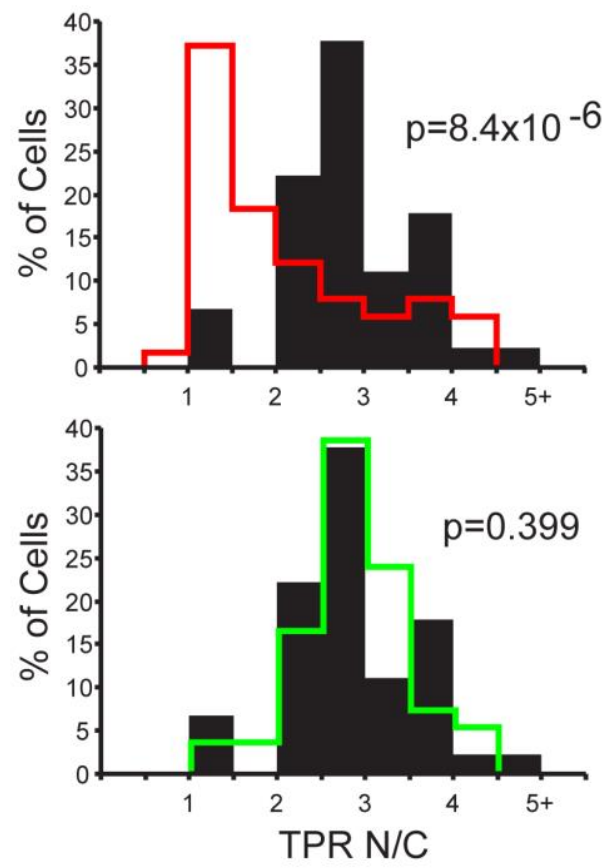
NLS-Ubc9 could restore H3K9me3 levels. By IF microscopy, HA-progerin transfected into HeLa cells with WT Ubc9 caused a statistically significant reduction of H3K9me3 levels (Fig 7 A, middle). However the cotransfection of HA-progerin with NLS-Ubc9 restored nuclear levels of $\mathrm{H} 3 \mathrm{~K} 9 \mathrm{me} 3$ significantly (Fig $7 \mathrm{~A}$, bottom). Our data suggest that the reduced level of $\mathrm{H} 3 \mathrm{~K} 9 \mathrm{me} 3$ induced by progerin expression is a consequence of either a Ran gradient disruption, a reduced level of Ubc9 function in the nucleus, or a combination of both mechanisms. 
Figure 7. Nuclear localization of Ubc9 restores H3K9me3 levels in cells expressing progerin. (A) IF localization of HA-progerin and nuclear H3K9me3 in cells cotransfected with Ubc9. Scale bar, $20 \mu \mathrm{m}$. (B) Histograms showing H3K9me3 levels (bin size $=1$ ) in HA-positive cells expressing engineered forms of Ubc9. "Copyright @ American Society for Microbiology, [Mol Cell Biol. 2011 August; 31(16): 3378-3395]" 
Figure 7

A

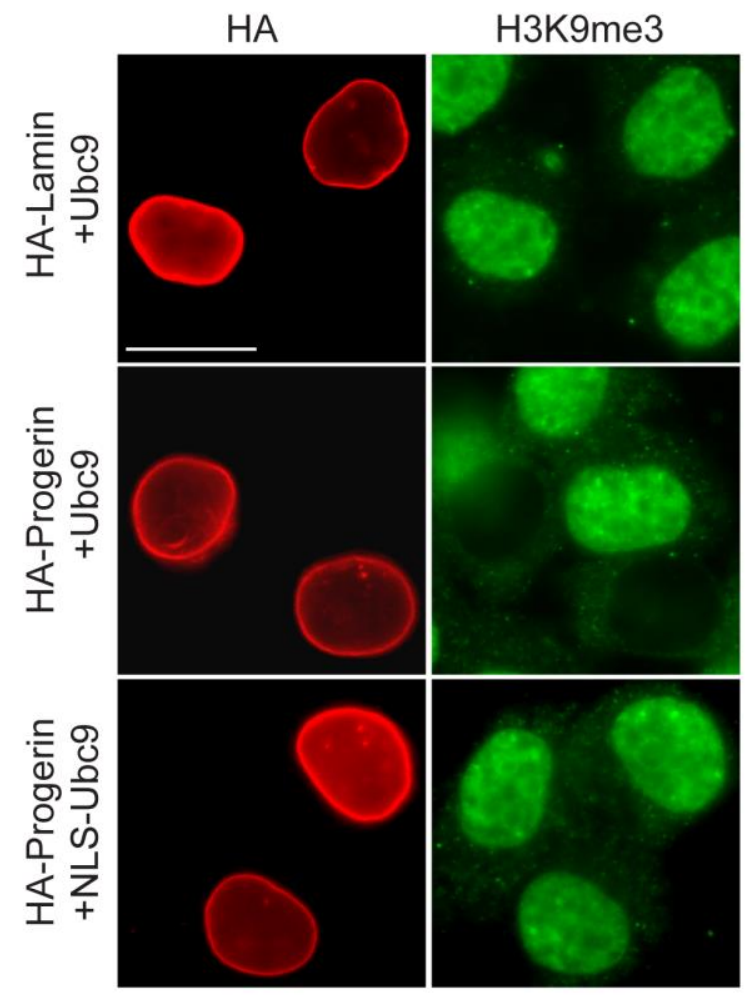

B

Lamin A Progerin Progerin

+Ubc9 +Ubc9 +NLS-Ubc9
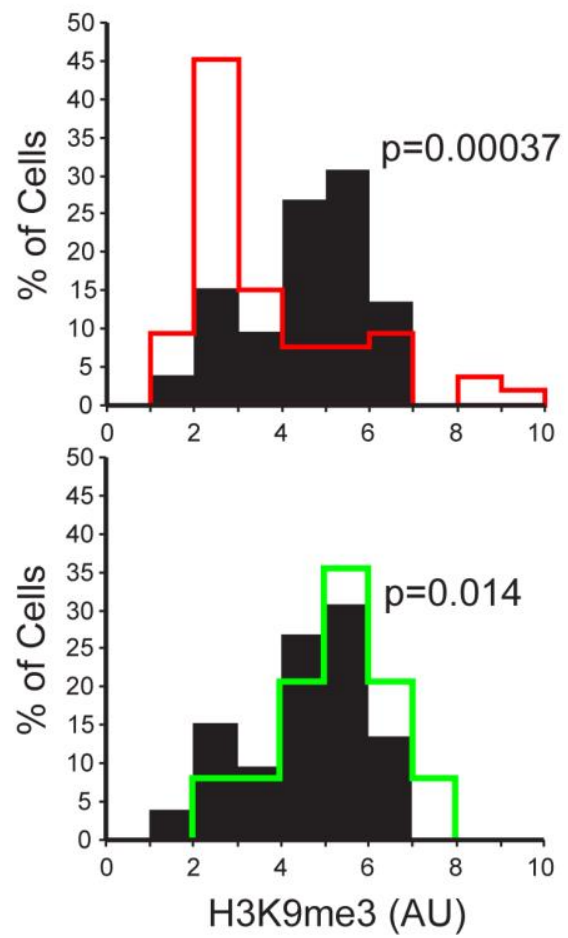


\section{DISCUSSION}

The fact that HGPS arises out of a mutation in the Lamin A gene, is well established (De Sandre-Giovannoli et al., 2003; Eriksson et al., 2003a). However the mechanisms that connect the most upstream event, which is the perturbation of the lamina, to the more downstream events like gene expression changes or global alterations in chromatin architecture, are still elusive. The goal of this study was to identify pathways that bridge defects in the lamina to the changes in chromatin that take place in HGPS. The disruption of the Ran gradient and the reduction of nuclear SUMOylation are two such observations that could possibly act as intermediate steps in the disease progression process. With the aim of ordering these two events, we showed that disruption of nuclear SUMOylation can disrupt the Ran gradient, indicating that nuclear SUMOylation is an upstream regulator of the Ran gradient. Since Ubc9 is the sole SUMO E2, we were interested in the localization of Ubc9 and interestingly both in HGPS cells as well as Progerin transfected cells, Ubc9 was mislocalized from the nucleus to the cytoplasm, and this was correlated with the Ran gradient disruption. Restoring the localization of Ubc9 in the context of Progerin, restored nuclear SUMOylation as well as the Ran gradient. However a catalytically dead mutant of Ubc9 when forced into the nucleus did not rescue nuclear SUMOylation or the Ran gradient. Thus a specific SUMOylation event carried out by Ubc9 in the nucleus is responsible for maintaining the Ran gradient. In addition to restoring the Ran gradient, Ubc9 could also rescue the nuclear import of nucleoporin TPR, whose import is defective in HGPS. Thus the nuclear import of certain nuclear proteins that depend on the Ran gradient, is regulated by nuclear SUMOylation carried out by Ubc9 (Figure 8). 
Figure 8. Working model for how progerin disrupts the Ran gradient in HGPS. The expression of the progerin form of lamin A in HGPS inhibits Ubc9 import, which results in reduced levels of nuclear SUMOylation by SUMO2/3, reduced RCC1 function, and a disruption of the Ran protein gradient. Although classical importin $\beta$-dependent import is largely unaffected by progerin, the nuclear import of TPR is defective because of its sensitivity to the Ran protein gradient. Loss of H3K9 trimethylation in HGPS may be downstream of the Ran gradient disruption, due to defective import of methyltransferases that maintain $\mathrm{H} 3 \mathrm{~K} 9$ trimethylation. It could also be a direct effect of reduced nuclear SUMOylation that alters chromatin structure and other chromatin modifications that ultimately affect H3K9-me3. Thus nuclear SUMOylation may be a central player in the initiation of the disruptive signals that allow the cellular progression of HGPS. 
Figure 8

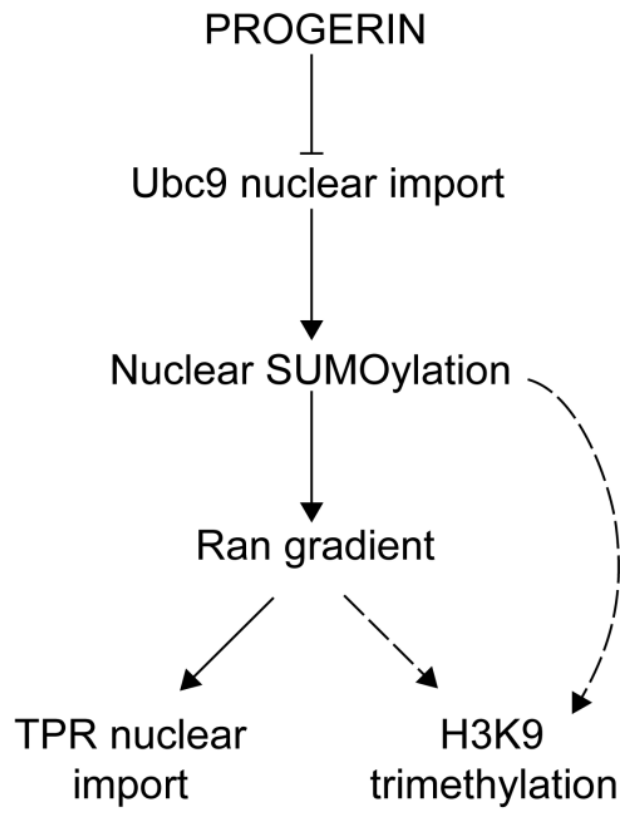


The mislocalization of Ubc9 might explain some of the epigenetic changes associated with HGPS. SUMOylation by Ubc9 regulates chromatin structure by modifying histones and chromatin-remodeling enzymes, resulting in chromatin compaction and gene repression (Shiio and Eisenman, 2003; Uchimura et al., 2006; Yang et al., 2007b). The reduced levels of the repressive chromatin mark H3K9me3 in HGPS and in HeLa cells expressing progerin, and the restoration of H3K9me3 levels upon the forcing of Ubc9 into the nucleus, suggest that reduced nuclear Ubc9 levels in HGPS might be responsible for the chromatin structural alterations occurring in HGPS. The rescue of $\mathrm{H} 3 \mathrm{~K} 9 \mathrm{me} 3$ levels could also be due to the rescue of the Ran gradient by NLS-Ubc9. Since Ubc9 has been shown to be imported into the nucleus by the Ran regulated import factor Importin13, the mislocalization of Ubc9 in HGPS could be a Ran dependent import defect (Mingot et al., 2001a). Thus the pathway of SUMOylation regulating the Ran gradient could include a positive-feedback loop wherein the the intact Ran gradient also regulates Ubc9 nuclear import. Progerin could disrupt the nuclear transport machinery by a yet unknown mechanism leading to the cytoplasmic localization of Ubc9. For example, Progerin could alter the expression of a factor that regulates the Ran gradient which when disrupted causes Ubc9 import defects, leading to a loss of nuclear SUMOylation and further loss of the Ran gradient. Regardless of which occurs first, a detailed insight into the mechanism of how the defects in the SUMOylation machinery are linked with the loss of the Ran gradient and how Progerin contributes towards these changes, is paramount towards the understanding of how HGPS progresses at the cellular level. 


\section{Chapter 3. A CENTRAL ROLE OF THE RAN GTPASE SYSTEM IN THE DEVELOPMENT OF SUMOYLATION DEFECTS AND OXIDATIVE STRESS IN HGPS}

\section{SUMMARY}

We recently demonstrated a loss of the Ran protein gradient and mis-localization of the SUMO E2 Ubc9 in HGPS which causes a loss of global nuclear SUMOylation. Here we establish that the mislocalization of Ubc9 in HGPS is a Ran dependent import defect. A gradually growing concept in the field of HGPS is oxidative stress. Here we show that the disruption of the Ran gradient also signals to the redox machinery and increases cellular ROS levels. To test whether ROS can in turn affect the cellular distribution of Ran and $\mathrm{Ubc9}$, we treated cells with $\mathrm{H}_{2} \mathrm{O}_{2}$ and both were rapidly mislocalized. Cells pre-adapted to Oxidative stress, when treated with $\mathrm{H}_{2} \mathrm{O}_{2}$ or when transfected with Progerin, did not have Ran or Ubc9 defects, indicating that increased cellular ROS levels in HGPS may also work upstream of Ran and Ubc9. However time course experiments using a Progerin mimetic revealed the ROS induction to be a biphasic phenomenon. Although the initial burst of ROS and the onset of Ran gradient disruption were temporally indistinguishable events, the secondary ROS induction was preceded by a Ran gradient disruption. Thus we highlight the central role of the Ran system in the initiating event following lamina perturbation, which by unleashing an oxidative stress response on one hand and global de-SUMOylation on the other, fosters the development of the cellular phenotypes in HGPS. 


\section{INTRODUCTION}

The nuclear lamina is a network of proteins proximal to the inner nuclear membrane that plays major roles in defining nuclear structure and function (Goldman et al., 2002). The most abundant components of the lamina are the A-type and B-type lamins, intermediate filament proteins that assemble into polymers (Goldman et al., 2002). The lamins interact with an expanding group of membrane-associated and nucleoplasmic proteins, and chromatin, to form macromolecular assemblies that regulate nuclear events including replication and transcription (Schirmer and Gerace, 2005). Studies over the past decade have revealed that mutations in genes encoding the lamins, particularly lamin A, are causal to a broad spectrum of human disorders termed laminopathies, which include cardiomyopathy, lipodystrophy, and certain types of muscular dystrophy (Mounkes et al., 2003). Remarkably, different mutations within the single gene encoding lamin $A(L M N A)$ give rise to disorders that can be manifest in a cell, tissue, and pathology-specific fashion (Mounkes et al., 2003).

One of the most highly studied laminopathies is Hutchinson-Gilford Progeria Syndrome (HGPS), a premature aging syndrome which is caused by a de novo mutation in LMNA. The LMNA mutation results in utilization of a cryptic pre-mRNA splice site and generation of a transcript that encodes the Progerin form of Lamin A that is missing 50 amino acids near its C-terminus (De Sandre-Giovannoli et al., 2003; Eriksson et al., 2003a). The Progerin form of Lamin A has a significant defect in processing, which is the basis of its dominant negative effects on the cell (De SandreGiovannoli et al., 2003; Eriksson et al., 2003a). WT Lamin A is farnesylated on a C- 
terminal CAAX motif, attached to the membrane, and subsequently proteolytically cleaved by Zmpste24 on the amino terminal side of Leu647. Thus, fully processed lamin A is not directly attached to the nuclear membrane (Goldman et al., 2002). Progerin is farnesylated, but it remains constitutively anchored to the nuclear membrane because the Zmpste24 cleavage site is within the 50 amino acid segment that is absent in Progerin (De Sandre-Giovannoli et al., 2003; Eriksson et al., 2003a). The results of a clinical trial using farnesyl transferase inhibitors to prevent membrane attachment of Progerin show moderate improvements in some of the anatomical and physiological malformations of HGPS (Gordon et al., 2012). While it is clear that constitutive attachment of lamin A is responsible for the changes associated with Progeria, the mechanisms that mediate the changes at the cellular and tissue levels remain a subject of investigation. One common phenotype reported for cells over-expressing Progerin is aberrant nuclear morphology including nuclear membrane blebbing (Goldman et al., 2004). A second phenotype of cells expressing Progerin is reduced levels of epigenetic marks on histone $\mathrm{H} 3$ that are associated with gene repression (Shumaker et al., 2006b) (Scaffidi and Misteli, 2006). Progerin, which is highly concentrated at the nuclear membrane, elicits epigenetic changes throughout the nucleus (Shumaker et al., 2006b) (Scaffidi and Misteli, 2006). This observation implies a dominant effect of nuclear architecture at the nuclear periphery that could have global effects on the nucleus.

We recently described phenotypic changes in HGPS cells that are associated with alterations in the nuclear transport machinery (Kelley et al., 2011). Nuclear import and export are regulated by the Ran GTPase, which rapidly shuttles between the nucleus and cytoplasm as part of its regulatory function (Pemberton and Paschal, 
2005). Under steady state conditions Ran has a predominantly nuclear distribution, and the difference in the concentration of Ran between the nucleus and cytoplasm is referred to as the Ran protein gradient (Bischoff et al., 1994a; Bischoff and Ponstingl, 1991; Görlich et al., 2003; Kelley and Paschal, 2007; Paschal, 1995; Pemberton and Paschal, 2005; Smith et al., 1998a). We discovered that the Ran nuclear to cytoplasmic ratio $(\mathrm{N} / \mathrm{C})$ is significantly reduced in HGPS cells.

One of the proteins whose nuclear localization is inhibited in HGPS is Ubc9, the E2 for SUMOylation (Geiss-Friedlander and Melchior, 2007; Kelley et al., 2011). Ubc9 is imported into the nucleus by Importin13, and following its translocation into the nucleus, the Ubc9-Importin13 complex is disassembled by RanGTP (Mingot et al., 2001a). Working in conjunction with Uba2/Aos1 E1 enzyme, Ubc9 either directly or with the assistance of E3 enzymes, conjugates SUMO to target proteins (Geiss-Friedlander and Melchior, 2007). SUMOylation regulates diverse pathways in the nucleus with roles in transcription, replication, and DNA repair (Geiss-Friedlander and Melchior, 2007). Ubc9 shuttles between the nucleus and the cytoplasm (Our unpublished data), and SUMOylation is known to regulate important events in the cytoplasm as well (GeissFriedlander and Melchior, 2007). There is also a pool of Ubc9 stably associated with the nucleoporin RanBP2 where it contributes to the SUMOylation of proteins associated with the nuclear pore complex (NPC) (Zhang et al., 2002). In our initial study it was not clear how Progerin triggers disruption of the Ran gradient, though loss of SUMOylation induced changes in the nuclear mobility of the nucleotide exchange factor RCC1 suggested a possible deficiency in GTP-GDP exchange on Ran (Kelley et al., 2011).

Loss of the Ran gradient has been linked with various kinds of cellular stresses 
like hyperosmotic stress (Kelley and Paschal, 2007) and oxidative stress(Kodiha et al., 2004; Miyamoto et al., 2004a; Yasuda et al., 2006). Reactive Oxygen Species (ROS) comprise a diverse variety of chemical derivatives of molecular oxygen that are by products of cellular metabolism (Finkel and Holbrook, 2000). Of these, the superoxide or hydroxyl radicals, are extremely unstable, whereas hydrogen peroxide is freely diffusible and has a relatively longer half-life (Finkel and Holbrook, 2000). ROS accumulation can have damaging effects on cellular components and hence there is a battery of enzymes like Superoxide Dismutase (SOD) and Catalase, that metabolize ROS and keep Oxidative stress to a minimum (Finkel and Holbrook, 2000).

In the present study we establish that our previously identified mislocalization of Ubc9 in HGPS (Chapter 2) is a Ran dependent import defect. A disruption of the Ran gradient also signals to the redox machinery and increases cellular ROS levels, which in turn can inhibit the nuclear import of Ubc9. Thus we highlight the central role of the Ran system in the development of the cellular phenotypes in HGPS. 


\section{MATERIALS AND METHODS}

\section{Cell culture and treatments}

Primary human fibroblasts from HGPS patients (AGO1972, AG11498, and AGO3199) and a clinically normal father (AGO8469) of an HGPS patient were obtained from the Coriell Cell Repository (Camden, NJ). These are designated HGPS 1972, HGPS 1498, HGPS 3199 and Normal 8469 respectively. Primary fibroblasts were grown at $37^{\circ} \mathrm{C}$ in $5 \% \mathrm{CO}_{2}$ in minimal essential medium (MEM) (Gibco) containing $15 \%$ FBS (Hyclone), 1 \% MEM vitamin solution (HyClone), 1\% penicillin/streptomycin (Gibco) and $1 \mathrm{mM}$ sodium pyruvate (Gibco). The passage number for cells in various experiments was between 8 and 25. HeLa and GSN2 cells (HeLa cells stably transfected with GFP-Streptavidin-SV40 NLS)(Black et al., 1999) were grown at $37^{\circ} \mathrm{C}$ in $5 \% \mathrm{CO}_{2}$ in Dulbecco's modified Eagle's medium (DMEM) (Gibco/Invitrogen) containing 10\% FBS (Atlanta Biologicals). Cos7 cells were grown in DMEM with 10\% FBS. CHO cells (HA-1 and OC-14) were generously provided by Douglas Spitz (Spitz et al., 1988a). These were grown at $37^{\circ} \mathrm{C}$ in $5 \% \mathrm{CO}_{2}$ in MEM (Gibco), supplemented with $15 \%$ FBS (Atlanta Biologicals) and 1\% penicillin/streptomycin (Gibco). Hydrogen Peroxide $\left(\mathrm{H}_{2} \mathrm{O}_{2}\right)$ (Catalog number $\left.\mathrm{H}-1009\right)$ was purchased from Sigma and used at concentrations and time points mentioned in the figure legends. Lopinavir was obtained from the NIH AIDS Research and Reference Reagent Program (Germantown, MD) (catalog number 9481; a gift from D. Rekosh) and Selleckchem (Catalog no. S-1380). Normal 8469 fibroblasts were treated with $40 \mu \mathrm{M}$ lopinavir dissolved in DMSO for 3 days 
or for the indicated time points and processed for immunoblotting, IF microscopy, ROS measurements or ATP measurements.

\section{Plasmids and siRNA}

Plasmids encoding the WT and mutant forms of Lamin A (pCDNA3-HA-Lamin and pCDNA3-HA-Progerin respectively) were generated as outlined in (Kelley et al.). pCDNA3-HA-Progerin C611S mutant was engineered by A. Spencer as described in(Snow et al., 2013). pCMV5-FLAG-Ubc9 and pCMV5-FLAG-Ubc9-C93S were generously provided by David Wotton. Small interfering RNA (siRNA) against NTF2 was obtained from Santa Cruz Biotechnology (Santa Cruz, CA). Stealth siRNA against Lamin A (LMNA stealth HSS106094) was purchased from Invitrogen (Catalog number 1299001). siRNA against Nup358/RanBP2 was purchased from Invitrogen (Catalog number 10620310)

\section{Transfection of plasmids and siRNA}

The cells to be transfected with plasmids were plated and grown in 6 well plates to a density of about $60-80 \%$ for 24 hours, followed by transfection with Transfectin (Biorad) according to the manufacturer's instructions. Transfected cells were processed for immunofluorescence microscopy or immunoblotting after 24 hours. NTF2 siRNA was transfected by using Lipofectamine RNAiMAX (Invitrogen) according to the manufacturer's instructions for reverse transfection. GSN2 cells were plated on NTF2 siRNA in 60mm dishes, split onto glass coverslips at $24 \mathrm{~h}$ post-transfection, transfected with pCMV-Flag-Ubc9 24 hours post splitting on to coverslips, and grown for an additional $24 \mathrm{~h}$, before processing for IF microscopy. For NTF2 knockdown in normal 
Human (8469) fibroblasts, cells were transfected with NTF2 siRNA by reverse transfection directly on coverslips for immunofluorescence or glass bottom dishes (Mattek) for ROS measurements and allowed to grow for 72 hours prior to processing. For Lamin A knockdown in 8469 fibroblasts, cells were transfected with Lamin A siRNA by the reverse transfection protocol followed by LPV treatment after 24 hours. The cells were allowed to grow for an additional 72 hours before processing for immunoblotting, immunofluorescence or ROS measurements.

\section{Heterokaryon cell fusion assays}

For the Heterokaryon assays, Cos7 cells were left un-transfected or transfected using fugene reagent (Promega) 24 hours after plating, as per the requirements of the experiment. After 24 hours the donor and acceptor cells were co-seeded on coverslips and after allowing them to grow for an additional 24 hours, cells were washed with prewarmed PBS and fused with 50\% Polyethylene glycol (Roche). Following an additional wash step, cells were incubated in $10 \mu \mathrm{g} / \mathrm{ml}$ cyclohexamide for 30 minutes or 2 hours in a $37^{\circ} \mathrm{C}$ incubator. The cells were then fixed and processed for immunofluorescence microscopy. Heterokaryons containing 2 nuclei per cell were visualized.

\section{ATP measurements}

ATP measurements were done using ATPlite luminescence ATP detection assay system (Perkin Elmer product \# 6016943) as per manufacturer protocols. Normal 8469 fibroblasts were plated in 96 well plates in triplicate and DMSO or LPV was added as indicated in the figure legends. On the day of processing, simultaneous to the ATP measurement, cells from an identical experiment were counted by trypan blue exclusion 
technique on a hemocytometer and the luminescence intensities were normalized to the cell numbers so obtained. In the same experiment, immunofluorescence detection of Ran gradient disruption was performed to verify the efficacy of the LPV treatment.

\section{Antibodies}

The following antibodies were used: Ran (Mouse monoclonal antibody, catalog number 610341; BD Biosciences), Lamin A (Rabbit polyclonal antibody [pAb], catalog number PRB-113c; Covance), Ubc9 (Rabbit pAb, catalog number ab33044; Abcam), anti-FLAG epitope (Mouse MAb M2; Sigma), OctA anti-FLAG (Rabbit pAb Santa Cruz), RanGAP (Mouse MAb 21c7; Zymed), anti-HA (Mouse MAb 16B12 and Rabbit pAb Y11; both from Santa Cruz), prelamin A (Goat pAb, catalog number sc-6214; Santacruz) and Tubulin (Mouse MAb 1-A2 Sigma).

\section{Immunoblotting}

Cells were lysed using standard SDS-PAGE loading buffer. Extracts were sonicated prior to loading on SDS-polyacrylamide gels. SDS-PAGE and immunoblotting were performed by standard methods using primary antibodies, peroxidase-labeled secondary antibodies and detected by chemiluminescence using ECL reagent. For the immunodetection of Ubc9-Uba2 thioester, cells were lysed in standard SDS-PAGE loading buffer containing 20mM N-Ethylmaleimide (NEM), 20mM lodoacetamide and protease inhibitors. $100 \mathrm{mM}$ DTT was added wherever indicated in the figures.

\section{Immunofluorescence microscopy}

Cells growing on glass coverslips were washed three times in PBS, fixed with $3.7 \%$ formaldehyde for 20 min, washed three times in PBS again and permeabilized in 
$0.2 \%$ Triton X-100 for 5 min. Coverslips were blocked in IF microscopy blocking buffer (1X PBS, 2\% bovine serum albumin, 2\% FBS) for 1 hour or overnight for Ubc9.

Coverslips were then incubated in primary antibody diluted in IF microscopy blocking buffer overnight at $4^{\circ} \mathrm{C}$ or at room temperature in the case of Ubc9. The secondary antibodies for IF microscopy were diluted in blocking buffer and incubated for 1 hour. The antibodies used were fluorescein isothiocyanate (FITC)-labeled donkey anti-mouse, Cy3-labeled donkey anti-mouse, FITC-labeled donkey anti-rabbit, FITC-labeled donkey anti-goat and Cy3-labeled donkey anti-rabbit antibodies (all from Jackson Immunoresearch). Wide-field microscopy was performed with a Nikon Eclipse E800 upright microscope using a 40X 1.0 NA oil immersion objective and recorded with a Hamamatsu C4742-95 charge-coupled device camera using OpenLab software (Perkin Elmer). Confocal imaging was done on a Zeiss LSM 510 microscope with $40 \mathrm{X}$ and 100X 1.3 NA oil immersion objectives using Axiovision software (Carl Zeiss) and on a Zeiss LSM 710 microscope with 20X regular and 63X oil immersion objectives using ZEN software (Carl Zeiss).

\section{ROS measurements}

2'7' Dichlorofluoroscein Diacetate (DCF-DA) was purchased from Sigma (Catalog number 35845 ) and a stock solution of $5 \mathrm{mM}$ made in ethanol. Cells growing in glass bottom dishes (Mattek) were washed three times with serum free MEM (Gibco) to remove traces of serum and incubated with a 1:2000 dilution of stock in serum free MEM for 30 minutes at $37^{\circ} \mathrm{C}$ and $5 \% \mathrm{CO}_{2}$. The cells were then washed and mounted in RPMI buffered medium (Gibco) followed by visualization of green fluorescence at an 
excitation wavelength of $488 \mathrm{~nm}$ on a Zeiss LSM 710 confocal microscope equipped with a stage warmer, with a 10X objective using ZEN software (Carl Zeiss).

\section{Measurement of Mitochondrial potential}

JC-1 was purchased from Invitrogen (Catalog number T-3168) and a $5 \mathrm{mg} / \mathrm{ml}$ stock solution was made in DMSO. Cells growing in glass bottom dishes (Mattek) were washed three times with regular HGPS media (See cell culture and treatments), incubated with $0.3 \mu \mathrm{g} / \mathrm{ml}$ concentration of JC-1 in HGPS media for 1 hour at $37^{\circ} \mathrm{C}$ and 5 $\% \mathrm{CO}_{2}$. The cells were then washed and mounted in RPMI buffered medium (Gibco) followed by visualization on a Zeiss LSM 510-META confocal microscope equipped with a stage warmer, with a $40 \mathrm{X}$ objective using Axiovision software (Carl Zeiss). JC-1 is a fluorescent dye that accumulates in mitochondria in the monomeric form and emits green fluorescence. It utilizes the mitochondrial potential to form fluorescent $\mathrm{J}$ aggregates which emit red fluorescence (Invitrogen). JC-1 was excited at 488nm and both the monomeric and $\mathrm{J}$ aggregate forms of $\mathrm{JC}-1$ were simultaneously visualized using 505-530 (Green) and 585-615 (Red) long pass filters respectively. The ratio of red to green fluorescence was quantified as a readout for polarized/total mitochondria.

\section{Image quantification and statistical analyses}

Quantitative analysis of IF microscopy images was performed using Image J image processing software $(\mathrm{NIH})$. For most of the figures more than 50 cells each from at least three experiments were quantified by circling relevant areas namely the nucleus and the cytoplasm, measuring the mean fluorescent intensities and representing the data as a mean $\mathrm{N} / \mathrm{C}$ ratio (Nuclear to Cytoplasmic fluorescence 
intensities). For Ubc9 rim quantification (Fig $9 \mathrm{C}$ ) the mean Ubc9 fluorescence intensity was calculated for 5 uniformly spread selections along the rim and their average values were plotted. For ROS measurements cells were outlined and mean DCF fluorescent intensities for the whole cell were plotted. For the measurement of mitochondrial potential using JC-1, individual cells were outlined and the ratio of mean red to green fluorescence intensities for each cell was plotted. Data were binned using Excel (Microsoft) prior to plotting as histograms, with bin sizes stated in the figure legends or were represented as bar graphs with error bars using standard deviations. The " $t$ test: two sample assuming equal variances" function of Excel (Microsoft) was used to calculate $p$ values, and the type 2 two tailed $p$ value was used. 


\section{RESULTS}

\section{Progerin inhibits nucleo-cytoplasmic shuttling of the SUMO conjugating enzyme} Ubc9.

Previously we showed that Ubc9, the E2 that conjugates SUMO to target proteins, is mis-localized from the nucleus to the cytoplasm in fibroblasts from HGPS patients (Kelley et al., 2011). Mis-localization of Ubc9 can be induced in HeLa cells by transient transfection of Progerin, the mutant form of lamin A expressed in HGPS fibroblasts (Kelley et al., 2011); (Fig. 9A). Detection of endogenous Ubc9 by immunofluoresecence (IF) microscopy and biochemical analysis of Ubc9 by other groups indicates there are two distinct pools of the protein, a soluble pool that shuttles between the cytoplasm and nucleoplasm, and an insoluble pool associated with RanBP2 at the NPC (Saitoh et al., 2002; Saitoh et al., 1997). Visual inspection of HAProgerin-transfected cells suggested that the large reduction in nuclear Ubc9 levels was not accompanied by a similar change in NPC-associated Ubc9 (Fig. 9 A). We measured the levels of nuclear, cytoplasmic, and NPC-associated Ubc9 in Progerin and WT lamin A (HA-tagged) transfected cells. Under conditions where Progerin expression dramatically reduced the levels of Ubc9 in the nucleus, there was a statistically insignificant change in the levels of Ubc9 associated with the NPC (Fig $9 \mathrm{~B}, \mathrm{C}$ ). Thus, Progerin selectively affects the pool of Ubc9 undergoing nucleo-cytoplasmic transport. The dominant negative effect of Progerin on Ubc9 import could be explained by retaining Ubc9 in the cytoplasm, or by inactivating the import signal in Ubc9. We used heterokaryon assays to address these possible models. HeLa cells transfected with 
Figure 9. Progerin inhibits nucleo-cytoplasmic shuttling of the SUMO conjugating enzyme Ubc9. (A) Endogenous Ubc9 distribution (red) in cells transfected with HALamin A and HA-Progerin (green). (B) Histogram of Ubc9 N/C (Bin size=0.2) from HALamin (black) and HA-Progerin (red). (C) Histogram of Ubc9 fluorescence measured at the nuclear envelope (Bin size=25) in HA-Lamin (black) and HA-Progerin (red) transfected cells. (D) Heterokaryon cell fusion using HeLa cells transfected with HAProgerin (green) or CFP (blue). Endogenous Ubc9 (red) was also detected. 16 out of 20 Heterokaryons counted had the phenotype shown. (E) Heterokaryon cell fusion by fusing HA-Progerin (red) and Flag-Ubc9 (green) transfected Cos cells with untransfected cells. 17 out of 18 Heterokaryons counted had the phenotype shown. (F) Heterokaryon cell fusion using Cos cells transfected with HA-Progerin (Red). Ran is shown in Green. 21 out of 23 Heterokaryons counted had the phenotype shown. Scale bars $=20 \mu \mathrm{m}(\mathrm{A})$ and $10 \mu \mathrm{m}(\mathrm{D}),(\mathrm{E})$ and $(\mathrm{F})$. 
Figure 9
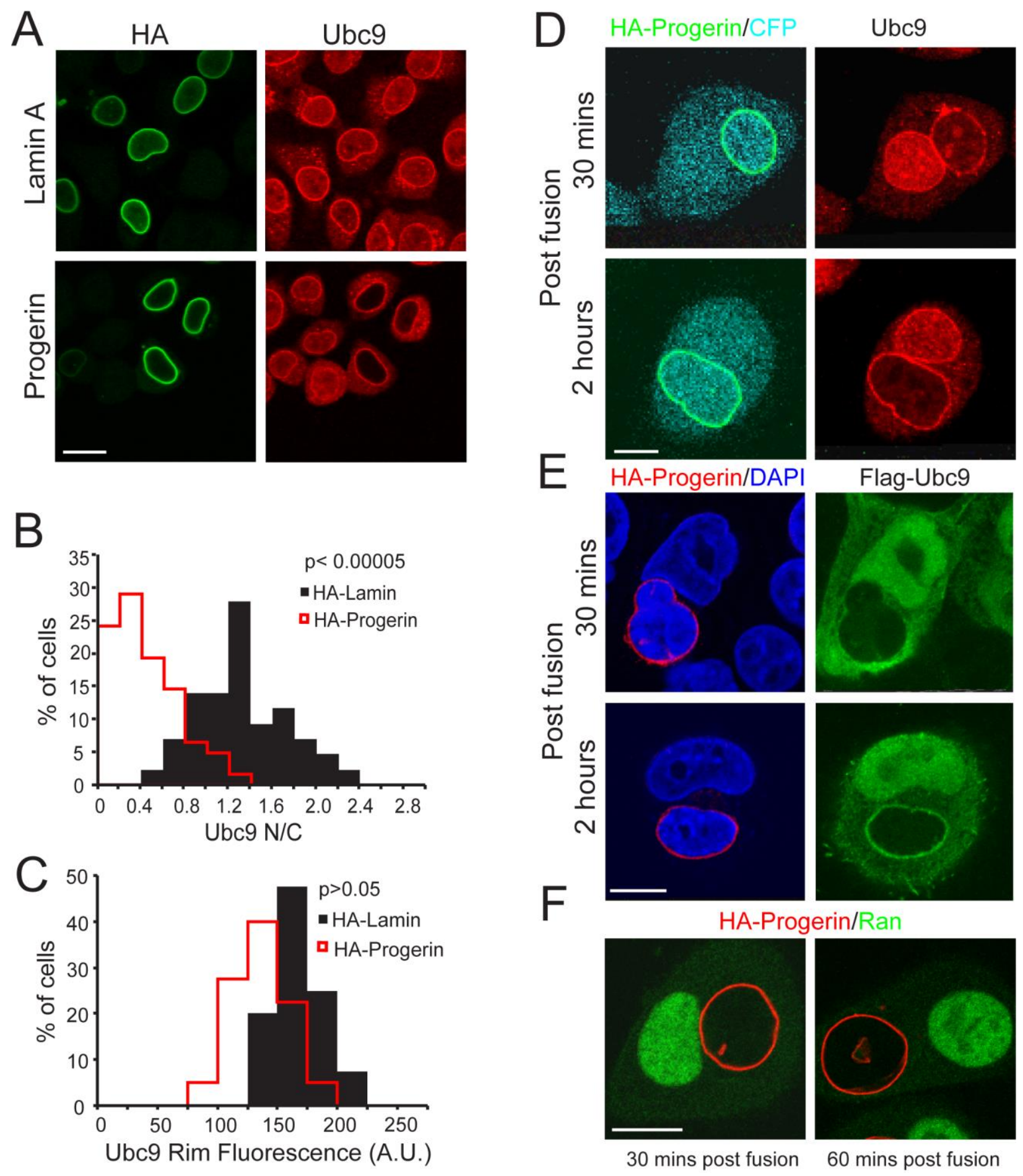
HA-Progerin were fused with HeLa cells transfected with CFP to mark the shared cytoplasm, and the localization of Progerin and endogenous Ubc9 determined by double label IF microscopy. In post-fusion heterokaryons, Ubc9 was concentrated in control nuclei, but failed to undergo import into nuclei that contained Progerin (Fig 9 D). The fact that the dominant negative effects of Progerin are not transduced within the cytoplasm of the heterokaryon argues strongly against a cytoplasmic signal being involved. Moreover, the fact that import in the Progerin-expressing nucleus was not restored by fusion with control cells suggests that the defect is associated with the nucleus and not the cytoplasm.

We tested for the import competence of Progerin mislocalized Ubc9 by cotransfecting HA-Progerin and Flag-tagged Ubc9, and subsequently fusing these cells with control cells. Flag-Ubc9 from the Progerin-expressing cell was fully competent for nuclear import into control nuclei, but failed to undergo import into the nucleus that contained Progerin (Fig. 9E). Flag-Ubc9 derived from the Progerin-expressing cell was incorporated into the NPC by 2 hrs (Fig. 9E), confirming the results described above (Fig. 9B, C) that Progerin inhibits Ubc9 import without disrupting its targeting to the NPC. As a control, Flag-Ubc9 was import competent in HA-Lamin transfected nuclei within the heterokaryons (Fig 10). The NPC-associated pool of Ubc9 detected by IF microscopy does not appear to be an intermediate in the Ubc9 nuclear import pathway. siRNA depletion of Nup358, which provides the binding site for Ubc9 on the cytoplasmic side of the NPC, did not affect nuclear levels of Ubc9 (Fig 11). We also examined the Ran distribution in heterokaryons. As expected, Ran was highly concentrated in the control nucleus but present at a much lower level in the nucleus from the Progerin- 
Figure 10. Heterokaryon cell fusion using Cos cells transfected with HA-Lamin (red) and Flag-Ubc9 (green). Blue represents DAPI stained nuclei. Scale bars $=10 \mu \mathrm{m}$. 
Figure 10

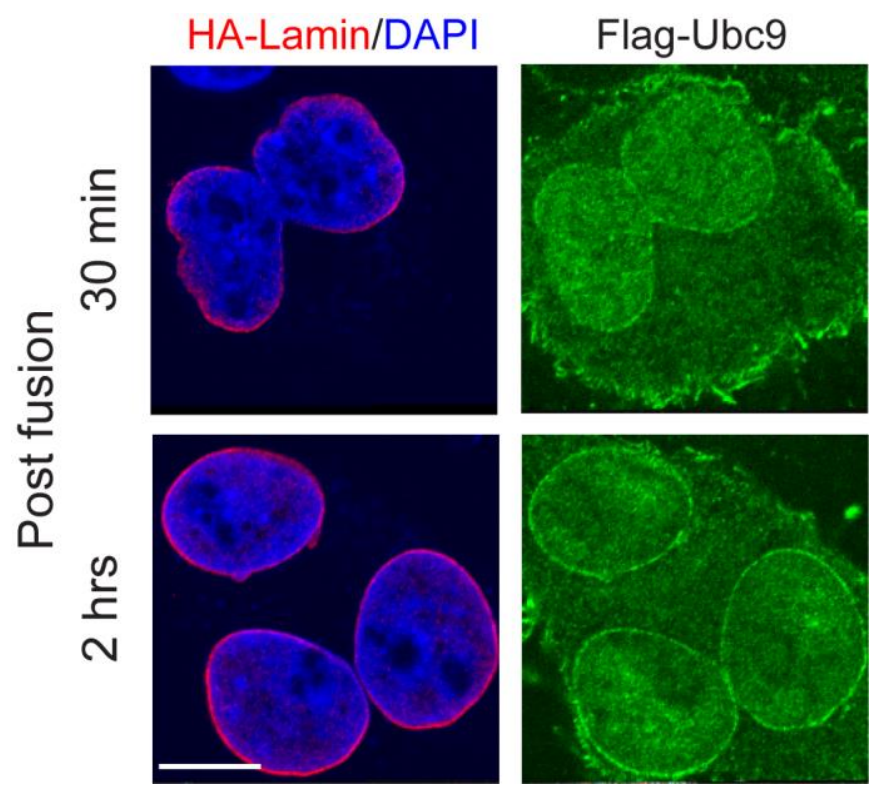


Figure 11. Nuclear import of Ubc9 does not require an interaction with Nup358 at the NPC. (A) siRNA mediated knockdown of Nup358 in HeLa cells and localization of RanGAP (green) and Ubc9 (red). RanGAP localization at the NPC was used as a surrogate marker for Nup358 expression, as RanGAP targeting to the NPC is strictly dependent on Nup358. (B) Histogram (Bin size $=0.25$ ) of Ubc9 N/C in control siRNA (black) and NTF2 siRNA (red) transfected cells. Scale bars $=20 \mu \mathrm{m}$. 
Figure 11
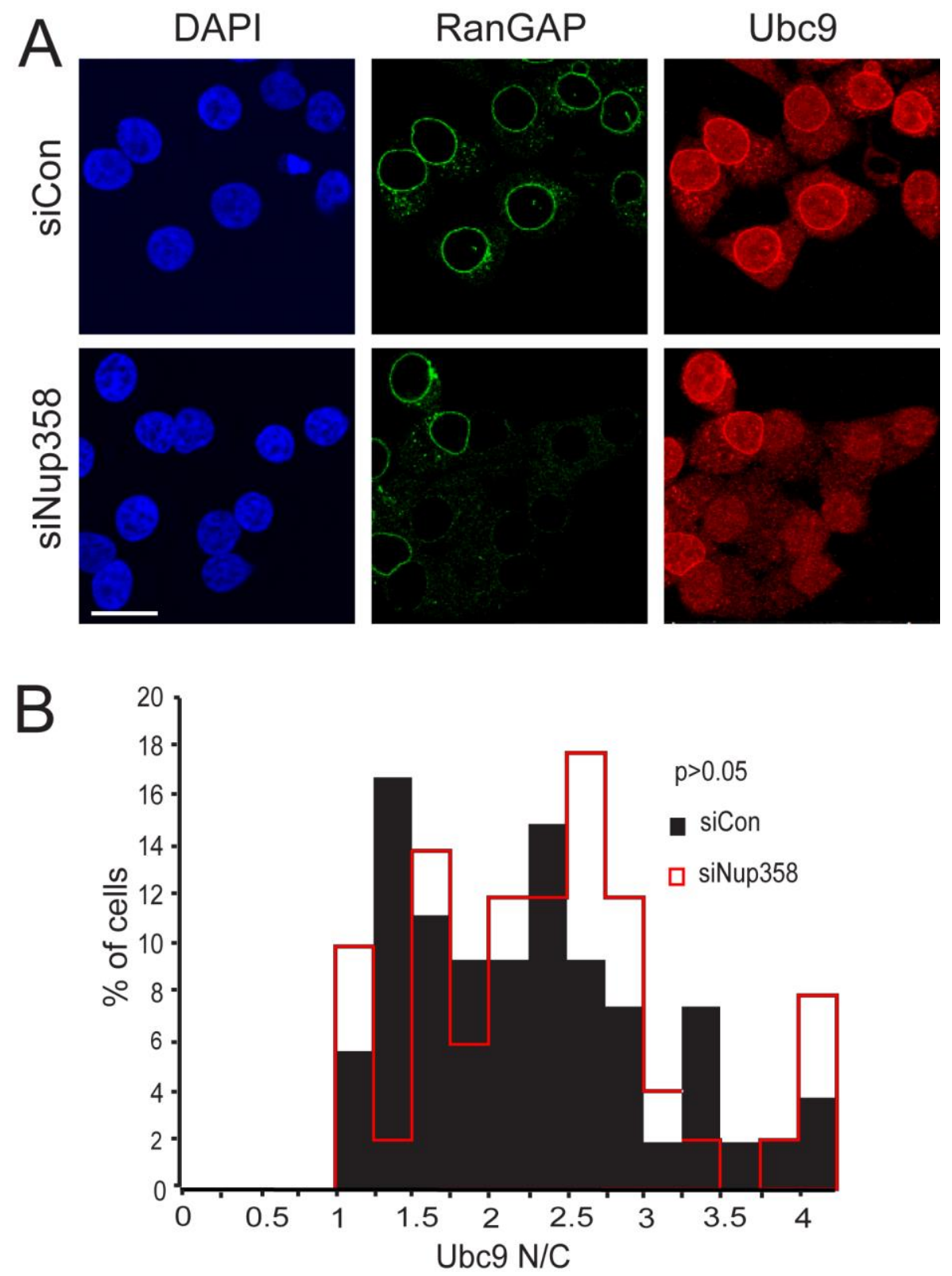
expressing cell (Fig. 9F). These experiments suggest that Progerin disruption of Ubc9 import is primarily a feature of the nucleus and not the cytoplasm, and the effect is not based on modifying the import competence of Ubc9.

\section{Fibroblasts from HGPS patients have elevated ROS}

Increased levels of ROS and oxidative damage are associated with aging (Finkel and Holbrook, 2000). ROS are elevated 1.6-fold in two fibroblast lines from HGPS patients (HGADFN167, HGADFN003), which also showed increased protein carbonyl content (Richards et al., 2010; Viteri et al., 2011). Increased ROS levels have also been demonstrated in human dermal fibroblasts transfected with Progerin (Pekovic et al., 2011).To ask whether elevated ROS might be common to HGPS fibroblasts, we measured ROS levels with the fluorescent indicator DCF-DA in three HGPS lines widely used in the field $(3199,1498,1972)$. All three lines had elevated levels of ROS compared to control fibroblasts from an unaffected parent of an HGPS patient (Fig. 12

A, B). The fact that $5 / 5$ HGPS lines examined to date have elevated ROS suggests that oxidative stress may be a common feature of HGPS cells. Oxidative stress has been shown to be associated with mitochondrial depolarization (Lemasters et al., 1998). Using JC-1, a fluorescent dye that produces red fluorescent aggregates in response to the mitochondrial membrane potential, we compared the mitochondrial membrane potential between normal and HGPS fibroblasts and found that HGPS cells unlike normal fibroblasts are unable to form the red fluorescent aggregates. This implies that HGPS cells have depolarized mitochondria indicative of mitochondrial dysfunction (Fig $13 \mathrm{~A}, \mathrm{~B})$. 
Figure 12. Fibroblasts from HGPS patients have elevated ROS. (A) ROS detected in primary fibroblasts from control (8469) and Progeria patients (1972, 1498 and 3199) using DCF. (B) Histograms comparing total ROS levels in control (Black bars) and Progeria patient fibroblasts (Red lines). Bin size $=100$. Scale bars $=20 \mu \mathrm{m}$. 
Figure 12

A

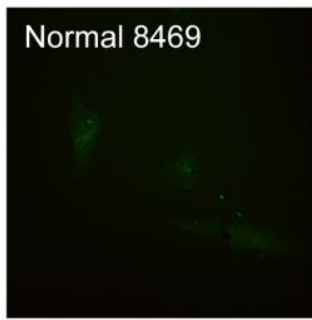

HGPS 1498

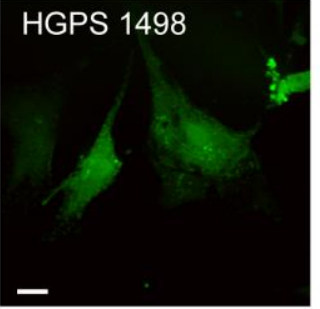

B
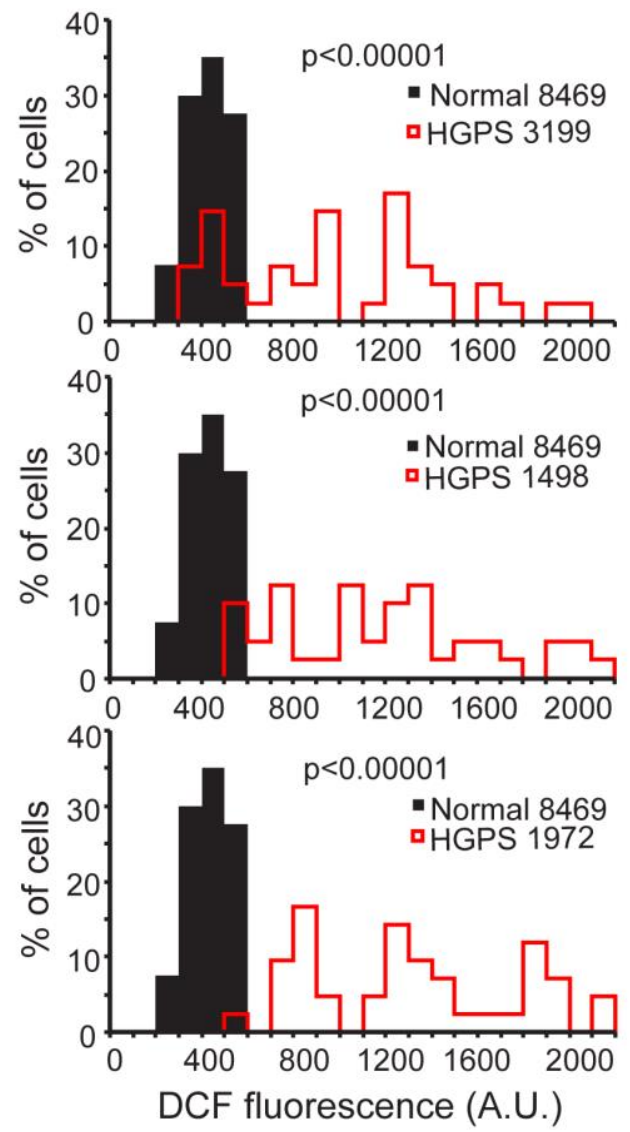
Figure 13. Mitochondria from HGPS fibroblasts are depolarized. (A) JC-1 fluorescence in fibroblasts from normal and HGPS patients. The total mitochondrial distribution is shown in green while the red dots are indicative of polarized mitochondria. (B) Histograms depicting the fractions of red/green intensity (polarized/total mitochondria) in HGPS cells (Red lines) and normal fibroblasts (Black bars). Bin size $=0.25$. Scale bars $=20 \mu \mathrm{m}$. 
Figure 13

A
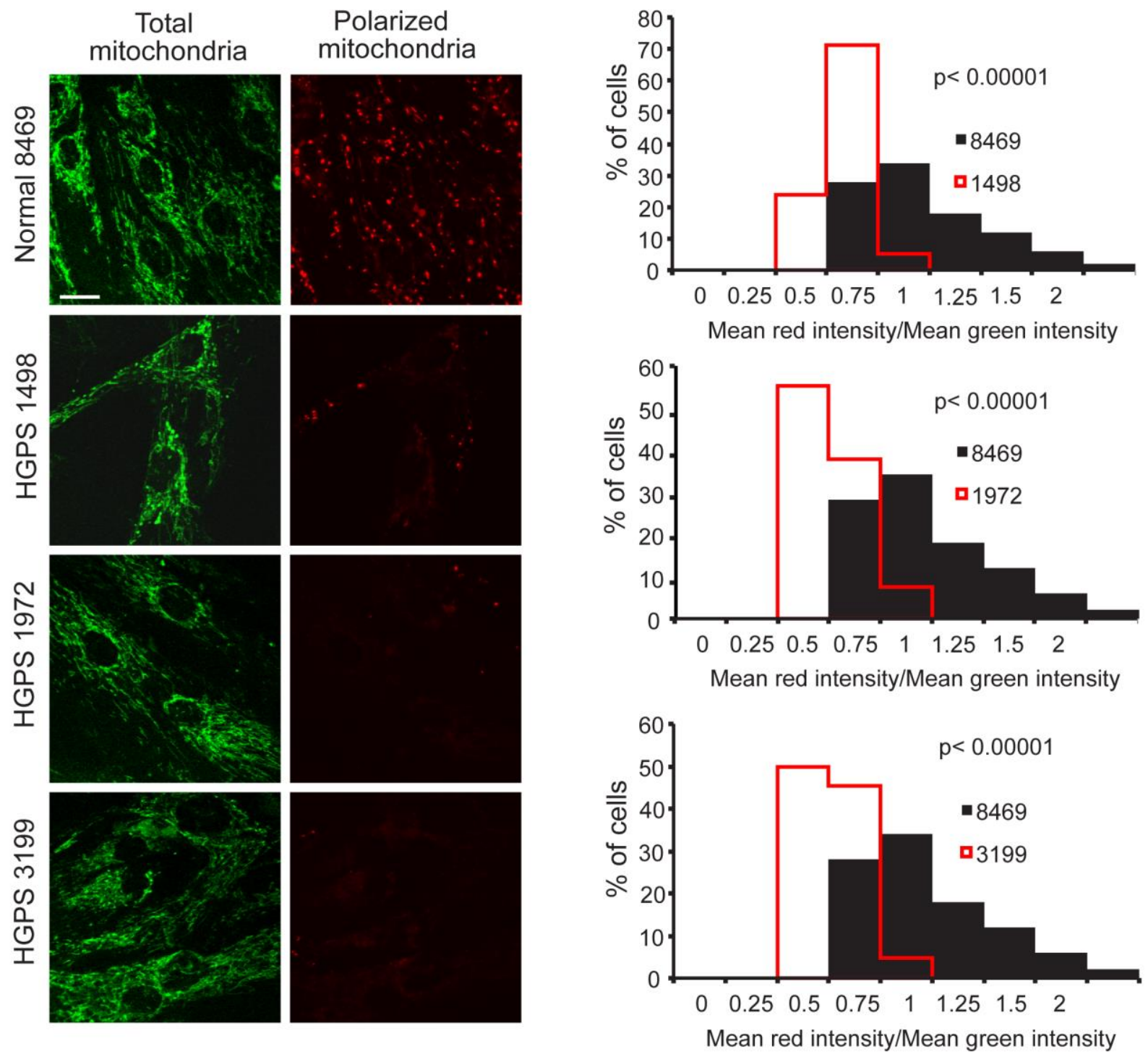


\section{Oxidative stress induced by $\mathrm{H}_{2} \mathrm{O}_{2}$ addition causes $\mathrm{Ubc} 9$ re-distribution to the cytoplasm.}

Oxidative stress induced by $\mathrm{H}_{2} \mathrm{O}_{2}$ results in a Ran distribution that is strikingly similar to that observed in HGPS cells, and in HeLa cells transfected with Progerin

(Czubryt et al., 2000; Miyamoto et al., 2004b) (Kelley et al., 2011). Given the elevation of ROS in HGPS cells, coupled with nuclear Ubc9 being upstream of the Ran gradient (Chapter 2), we considered whether ROS effects on the Ran gradient involve a mislocalization of Ubc9. The simple model is that Progerin expression elevates ROS levels, which mislocalizes Ubc9, which in turn disrupts the Ran gradient. To determine whether oxidative stress is sufficient to change Ubc9 distribution in the cell, we treated HeLa cells with a range $(0-400 \mu \mathrm{M})$ of $\mathrm{H}_{2} \mathrm{O}_{2}$ concentrations and examined endogenous Ubc9 by IF microscopy. Treating HeLa cells with $\mathrm{H}_{2} \mathrm{O}_{2}$ for 10 minutes was sufficient to markedly reduce the nuclear concentration of Ubc9, an effect that was clearly visible with $100 \mu \mathrm{M} \mathrm{H}_{2} \mathrm{O}_{2}$ and above (Fig $14 \mathrm{~A}$ ). Using biochemical approaches the Melchior group showed that in response to $\mathrm{H}_{2} \mathrm{O}_{2}, \mathrm{Ubc9}$ forms a heterodimer with the $\mathrm{E} 1 \mathrm{Uba} 2$ (Bossis and Melchior, 2006). The Ubc9-Uba2 disulfide product was detected from cells treated with as little as $50 \mathrm{uM} \mathrm{H} \mathrm{O}_{2}$ (Fig. 14 B). Higher concentrations of $\mathrm{H}_{2} \mathrm{O}_{2}(100$ and $200 \mu \mathrm{M} \mathrm{H} 2 \mathrm{O} 2$ ) further increased disulfide product formation (Fig $14 \mathrm{~B}$ ). To test whether the effect of $\mathrm{H}_{2} \mathrm{O}_{2}$ on Ubc9 localization is reversible, we treated HeLa cells with $200 \mu \mathrm{M}$ $\mathrm{H}_{2} \mathrm{O}_{2}$ for 10 mins, washed the cells, and added fresh media for 30 and 60 min to allow recovery. Under these conditions the nuclear levels of Ubc9 and Ran were reduced by $\mathrm{H}_{2} \mathrm{O}_{2}$ treatment, but nuclear levels of both proteins were recovered in most cells by 30 min (Fig 14 C). Immunoblotting for Ubc9 and Ran levels showed that they were 
Figure 14. Oxidative stress induced by $\mathrm{H}_{2} \mathrm{O}_{2}$ addition causes $\mathrm{Ubc} 9$ re-distribution to the cytoplasm. (A) Endogenous Ubc9 distribution detected by IF in HeLa cells treated for 10 minutes with the indicated concentrations of $\mathrm{H}_{2} \mathrm{O}_{2}$. (B) Ubc9-Uba2 disulfide detected by immunoblotting under non-reducing conditions. HeLa cells were treated for 10 minutes with the indicated concentrations of $\mathrm{H}_{2} \mathrm{O}_{2}$ immunoblotted for Ubc9. Gel samples were prepared without or with $100 \mathrm{mM}$ DTT, as indicated. (C) Endogenous Ubc9 (Red) and Ran (Green) in HeLa cells undergo reversible changes in distribution in response to oxidative stress. HeLa cells were treated for 10 min with $200 \mu \mathrm{M} \mathrm{H}_{2} \mathrm{O}_{2}$ and then allowed to recover in fresh media for 30 and $60 \mathrm{~min}$. Scale bars $=20 \mu \mathrm{m}$. 
Figure 14

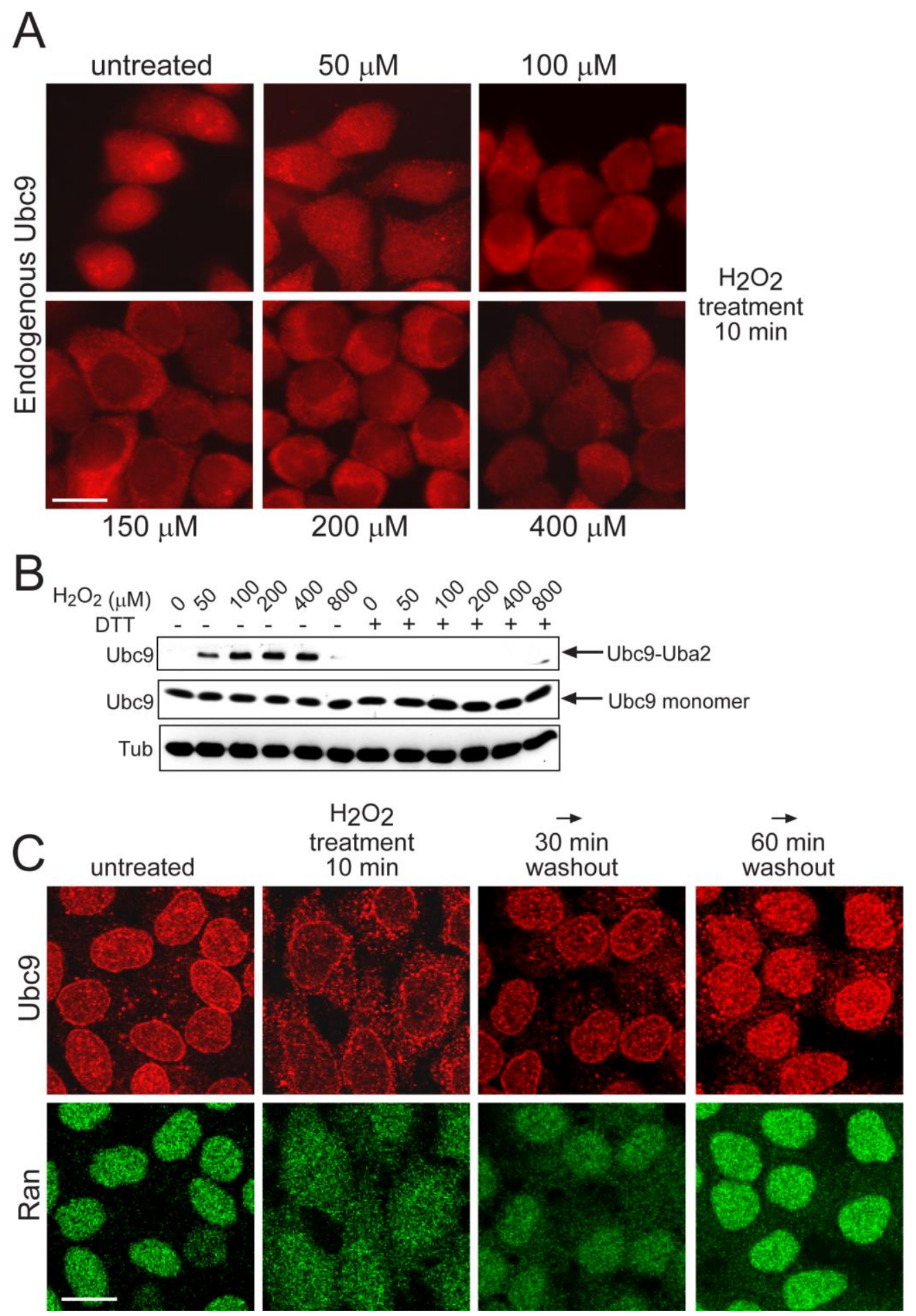


unchanged (Data not shown). Oxidative stress can therefore affect Ubc9 by two mechanisms namely heterodimer formation with Uba2 and altered sub-cellular localization through a nuclear transport-based mechanism.

\section{Nuclear localization of Ubc9 is resistant to the effects of $\mathrm{H}_{2} \mathrm{O}_{2}$ in $\mathrm{CHO}$ cells pre- adapted to oxidative stress.}

As an additional approach to determine whether Ubc9 localization is responsive to oxidative stress, we took advantage of a $\mathrm{CHO}$ cell line that is oxidative stressresistant (Spitz et al., 1988a). The OC-14 line was adapted to oxidative stress in culture, and stably over-expresses catalase $>10$-fold relative to the parental line HA-1 (Spitz et al., 1988b). In the HA-1 (Parental) cell line, the nuclear distribution of both Ubc9 and Ran was unaffected by $200 \mu \mathrm{M} \mathrm{H}_{2} \mathrm{O}_{2}$ but reduced significantly by $400 \mu \mathrm{M} \mathrm{H}_{2} \mathrm{O}_{2}$ (Fig. 15A). Nuclear localization of Ubc9 and Ran in the OC-14 (Resistant) cell line was unaffected by $400 \mu \mathrm{M} \mathrm{H}_{2} \mathrm{O}_{2}$, but both proteins were disrupted by $800 \mu \mathrm{M} \mathrm{H}_{2} \mathrm{O}_{2}$ (Fig. 15A). By immunoblotting, the Ubc9-Uba2 disulfide product was detected in HA-1 (Parental) cells treated with $\mathrm{H}_{2} \mathrm{O}_{2}$ (Fig. 15B). In contrast, the Ubc9-Uba2 disulfide was not detected in the OC-14 (Resistant) cells even with high concentrations of $\mathrm{H}_{2} \mathrm{O}_{2}$ (Fig. 15C), consistent with oxidative stress resistance of this line. Thus, oxidative stress resistance of the OC-14 cell line (defined originally in terms of viability); affords protection of Ubc9 and Ran from stress-induced changes in distribution and Ubc9 from disulfide formation with Uba2. 
Figure 15. Nuclear localization of Ubc9 is resistant to the effects of $\mathrm{H}_{2} \mathrm{O}_{2}$ in $\mathrm{CHO}$ cells pre-adapted to oxidative stress. (A) $\mathrm{H}_{2} \mathrm{O}_{2}$ effects on $\mathrm{Ubc9}$ and Ran distribution in $\mathrm{CHO}$ cells. Cells were treated with the indicated concentrations of $\mathrm{H}_{2} \mathrm{O}_{2}$ for 10 min, and endogenous Ubc9 and Ran were detected by IF. Scale bars $=20 \mu \mathrm{m}$. (B) and (C) Ubc9Uba2 disulfide formation in HA-1 and OC14 cells. Cells treated with different concentrations of $\mathrm{H}_{2} \mathrm{O}_{2}$ for 10 min were analyzed by immunoblotting. DTT was added to the samples wherever indicated. The electrophoretic position of the Ubc9-Uba2 disulfide (arrow) and a non-specific cross-reaction product (asterisk) are indicated. 
Figure 15

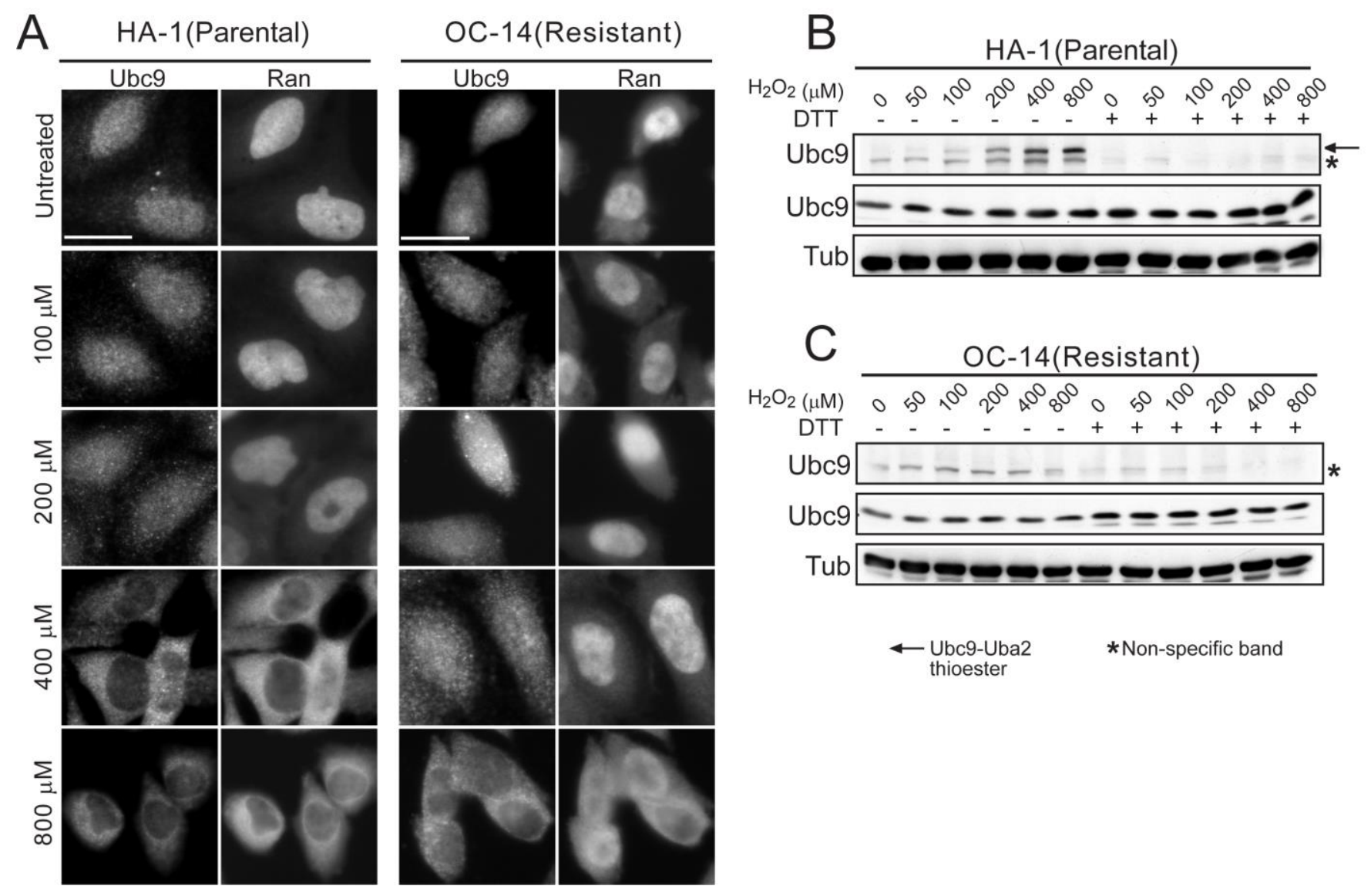




\section{Stress-resistant $\mathrm{CHO}$ cells resist the effects of Progerin on nuclear transport.}

We next used the HA-1 and OC-14 cell lines to test whether Progerin effects on

Ubc9 and Ran distribution are mediated by oxidative stress. If so, the OC-14 (Resistant) cells would be expected to resist the effects of Progerin expression. We transfected HAtagged Progerin and WT Lamin A into HA-1 (Parental) and OC-14 (Resistant) cells, and analyzed the samples by double label IF microscopy. In HA-1 (Parental) cells, transfected HA-Progerin disrupted the Ran gradient and lead to a striking reduction in nuclear levels of Ubc9 (Fig. $16 \mathrm{~A}$, upper panels). By contrast, in OC-14 cells the distribution of Ran and Ubc9 appeared to be unaffected by Progerin (Fig. 16 A, lower panels). Quantitative analysis confirmed that there is a significant difference in Ran and Ubc9 distribution in Progerin transfected HA-1 and OC-14 cells (Fig. 16 B, C).

\section{Ubc9 localization to the cytoplasm in response to Progerin and oxidative stress appears to be independent of cysteine 93 oxidation.}

$\mathrm{H}_{2} \mathrm{O}_{2}$ disrupted Ubc9 localization and induced Ubc9-Uba2 disulfide formation in both HeLa cells and the HA-1 (Parental) CHO cells (Fig 14, 15). In OC-14 (Resistant) $\mathrm{CHO}$ cells, however, Ubc9 distribution was disrupted with $800 \mu \mathrm{M} \mathrm{H}_{2} \mathrm{O}_{2}$ with no evidence of Ubc9-Uba2 disulfide formation (Fig 15). These observations led us to question whether loss of Ubc9 from the nucleus in response to Progerin and oxidative stress is linked to Ubc9-Uba2 disulfide formation, or whether these are independent events. To address this question, we used a form of Ubc9 that contains an amino acid substitution in the cysteine that forms the disulfide with Uba2 (C93S). Flag-Ubc9-C93S like Flag-Ubc9-WT, was redistributed from the nucleus to the cytoplasm in response to 
Figure 16. Stress-resistant $\mathrm{CHO}$ cells resist the effects of Progerin on nuclear transport. (A) Transfection of HA-Lamin and HA-Progerin into $\mathrm{CHO}$ cell lines followed by detection of endogenous Ubc9 and Ran by IF. Lamin A and Progerin expression were detected with anti-HA antibody. $(B, C)$ Histograms (Bin size $=0.5$ ) showing the Ran N/C and Ubc9 N/C in the HA-1(Parental) and OC-14(Resistant) cells transfected with Progerin. Scale bars $=20 \mu \mathrm{m}$. 
Figure 16
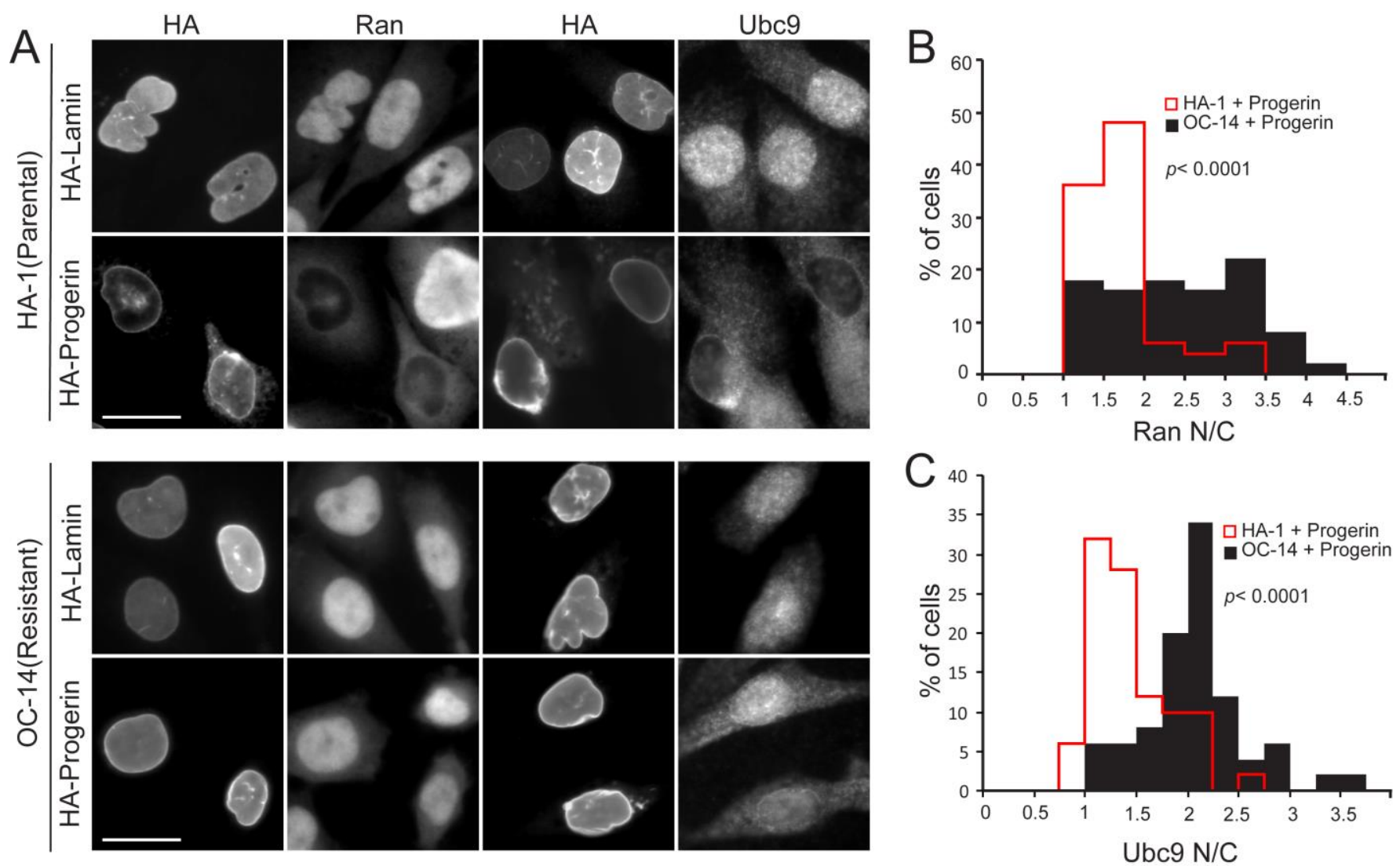
Progerin expression (Fig. 17 A) and to $\mathrm{H}_{2} \mathrm{O}_{2}$ treatment (Fig. 17 B). By quantitative analysis Progerin reduced the nuclear levels of Flag-Ubc9-C93S to the same extent as Flag-Ubc9-WT (Fig. 17 C, D). Thus, disulfide formation is not necessary for the changes in Ubc9 distribution in response to Progerin and $\mathrm{H}_{2} \mathrm{O}_{2}$. We next examined three HGPS cell lines $(1972,3199,1498)$ for the presence of the Ubc9-Uba2 disulfide. The Ubc9Uba2 disulfide was not detected (Fig $17 \mathrm{E}$ ), despite the fact it can be induced by $\mathrm{H}_{2} \mathrm{O}_{2}$ treatment of passage number-matched control (8469) fibroblasts (Fig 18). Our data indicate that Progerin and oxidative stress effects on Ubc9 distribution can occur independently of Ubc9 oxidation and disulfide formation with Uba2.

\section{Nuclear localization of Ubc9 requires a Ran protein gradient.}

Loss of $\mathrm{Ubc9}$ from the nucleus in response to $\mathrm{H}_{2} \mathrm{O}_{2}$ and Progerin expression could be a response to Ran gradient disruption under these conditions. In HeLa cells treated with $\mathrm{H}_{2} \mathrm{O}_{2}$, there is a simultaneous loss of Ran and Ubc9 from the nucleus detected within 2 min (Fig. 19 A, B). Plotting the Ubc9 N/C as a function of the Ran N/C revealed a strong correlation with or without treating cells with $\mathrm{H}_{2} \mathrm{O}_{2}$ (Fig. 19 C). Given a large number of cellular pathways can be affected by $\mathrm{H}_{2} \mathrm{O}_{2}$, we directly tested whether the level of nuclear Ran controls Ubc9 import by depleting the Ran import factor NTF2. For this experiment we used a HeLa cell line that stably expresses a GFP-NLS reporter protein (Black et al., 1999). Disruption of the Ran gradient by NTF2 depletion was sufficient to reduce the nuclear concentration of Ubc9 without a significant effect on nuclear localization of the GFP-NLS reporter (Fig. 19 D, E, F). The Ubc9 N/C was 
Figure 17. Ubc9 localization to the cytoplasm in response to Progerin and oxidative stress appears to be independent of cysteine 93 oxidation. (A) Localization of FlagUbc9 (WT and C93S mutant; green) in HeLa cells co-transfected with HA-Lamin or HAProgerin (red). (B) Localization of Flag-Ubc9 (WT and C93S mutant; green) in HeLa cells treated with $200 \mu \mathrm{M} \mathrm{H}_{2} \mathrm{O}_{2}$ for 10 min. Scale bars $=10 \mu \mathrm{m}$. (C, D) Histograms showing N/C values (bin size $=0.5$ ) of Flag-Ubc9 and Flag-Ubc9-C93S in cells expressing HA-Lamin (black) and HA-Progerin (red). (E) Immunoblotting for the Ubc9Uba2 disulfide in control (8469) and HGPS (1972, 1498 and 3199) fibroblasts. The electrophoretic position of the Ubc9-Uba2 disulfide (arrow) and a non-specific crossreaction (asterisk) are indicated. 
Figure 17

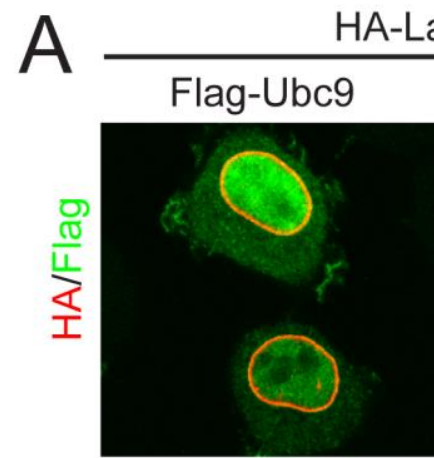

B

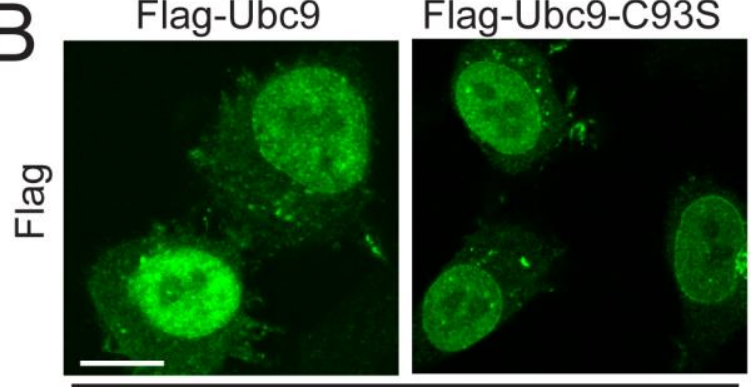

untreated

C

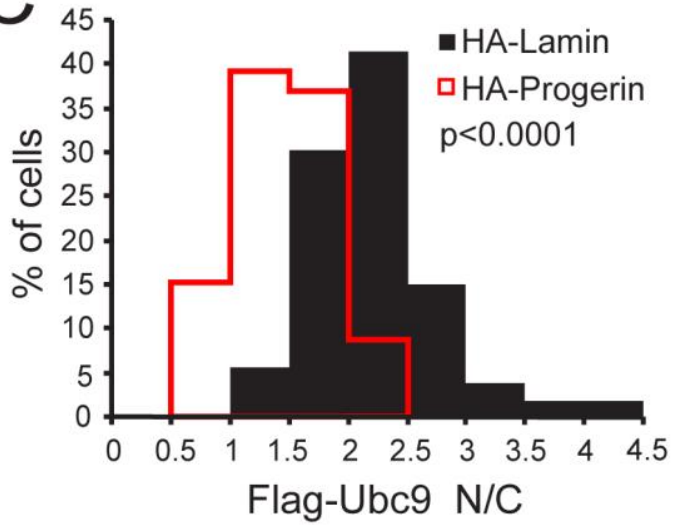

HA-Progerin

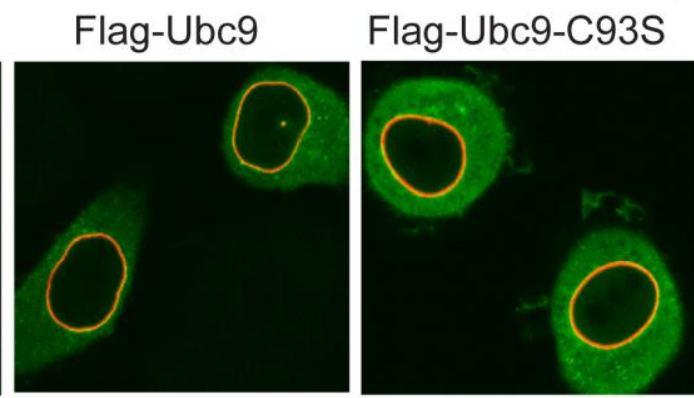

Flag-Ubc9

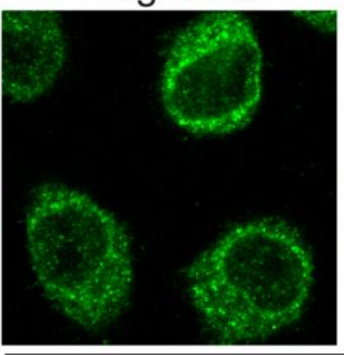

Flag-Ubc9-C93S

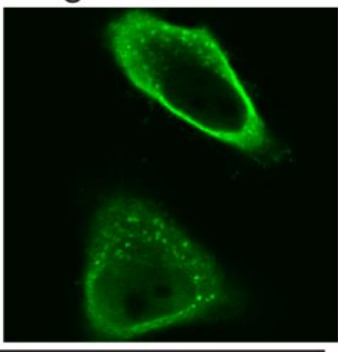

$\mathrm{H}_{2} \mathrm{O}_{2}(200 \mu \mathrm{M})$

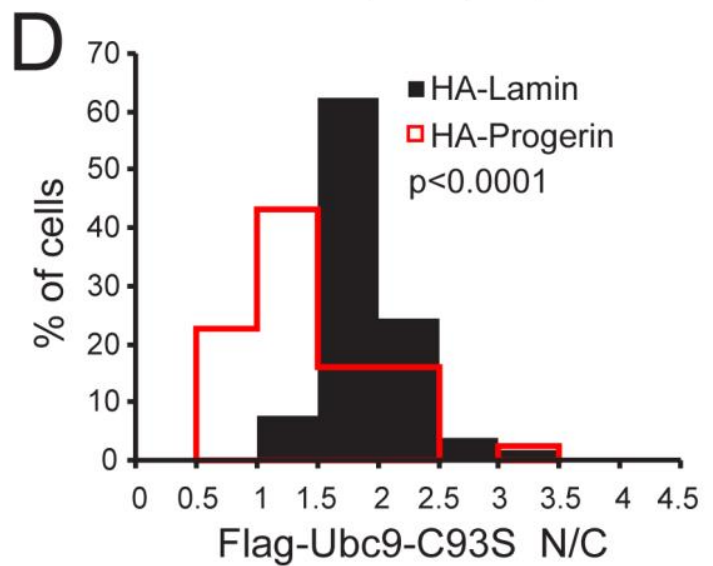

E

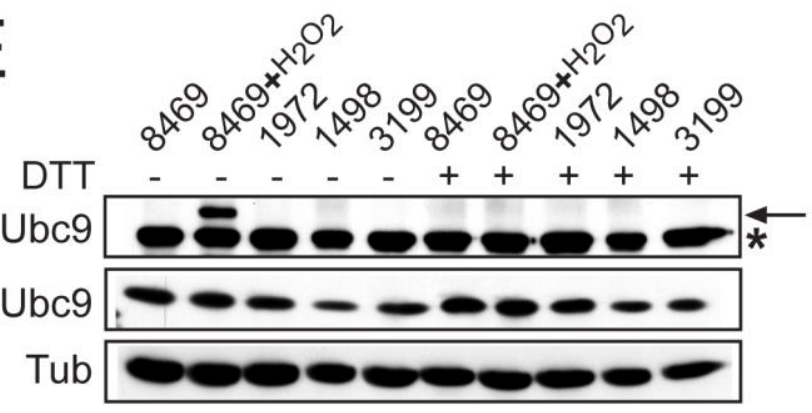

—Ubc9-Uba2 * Non-specific band 
Figure 18. Ubc9-Uba2 disulfide formation in 8469 fibroblasts. Cells treated with different concentrations of $\mathrm{H}_{2} \mathrm{O}_{2}$ for 10 min were analyzed by immunoblotting. Gel samples were prepared with or without $100 \mathrm{mM} \mathrm{DTT}$, as indicated. The electrophoretic position of the Ubc9-Uba2 disulfide (arrow) and a non-specific cross-reaction product (asterisk) are indicated. 
Figure 18

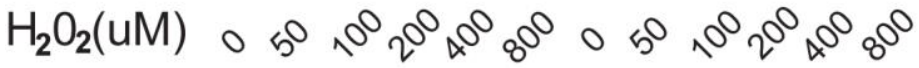

$$
\begin{aligned}
& \text { DTT - - - - }- \text { - + + + + }
\end{aligned}
$$

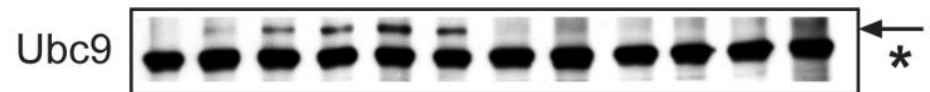

$$
\begin{aligned}
& \text { Ubc9 -- - - - - - - - - }
\end{aligned}
$$

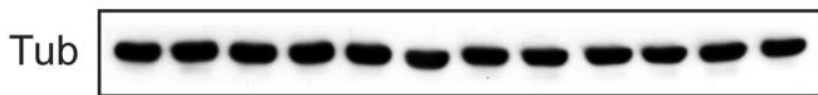


Figure 19. Nuclear localization of Ubc9 requires a Ran protein gradient. (A, B) Ubc9 and Ran N/C levels in HeLa cells treated with $200 \mu \mathrm{M} \mathrm{H} \mathrm{H}_{2}$ measured as a function of time. (C) XY Scatter plot of Ubc9 N/C and Ran N/C in untreated (black dots) and $\mathrm{H}_{2} \mathrm{O}_{2}-$ treated HeLa cells (red dots). (D) Disruption of the Ran gradient by siRNA depletion of NTF2 in GSN2 cells. HeLa cells (stably transfected with GFP-NLS; (Black et al., 1999) were co-transfected with siRNA and Flag-Ubc9, and imaged in three channels for endogenous Ran (purple), Flag-Ubc9 (red), and GFP (green) Scale bars $=20 \mu \mathrm{m}$. (E) Histogram (bin size $=0.25$ ) showing Flag-Ubc9 N/C from control siRNA (black) and NTF2 siRNA (red line) transfected cells. (F) Histogram (bin size=25) showing nuclear fluorescence intensities of GFP-NLS from control (black) and NTF2 siRNA (red) transfected cells. (G) XY scatter plot of Ubc9 N/C and Ran N/C in control siRNA (black dots) and NTF2 siRNA (red dots) cells. 
Figure 19
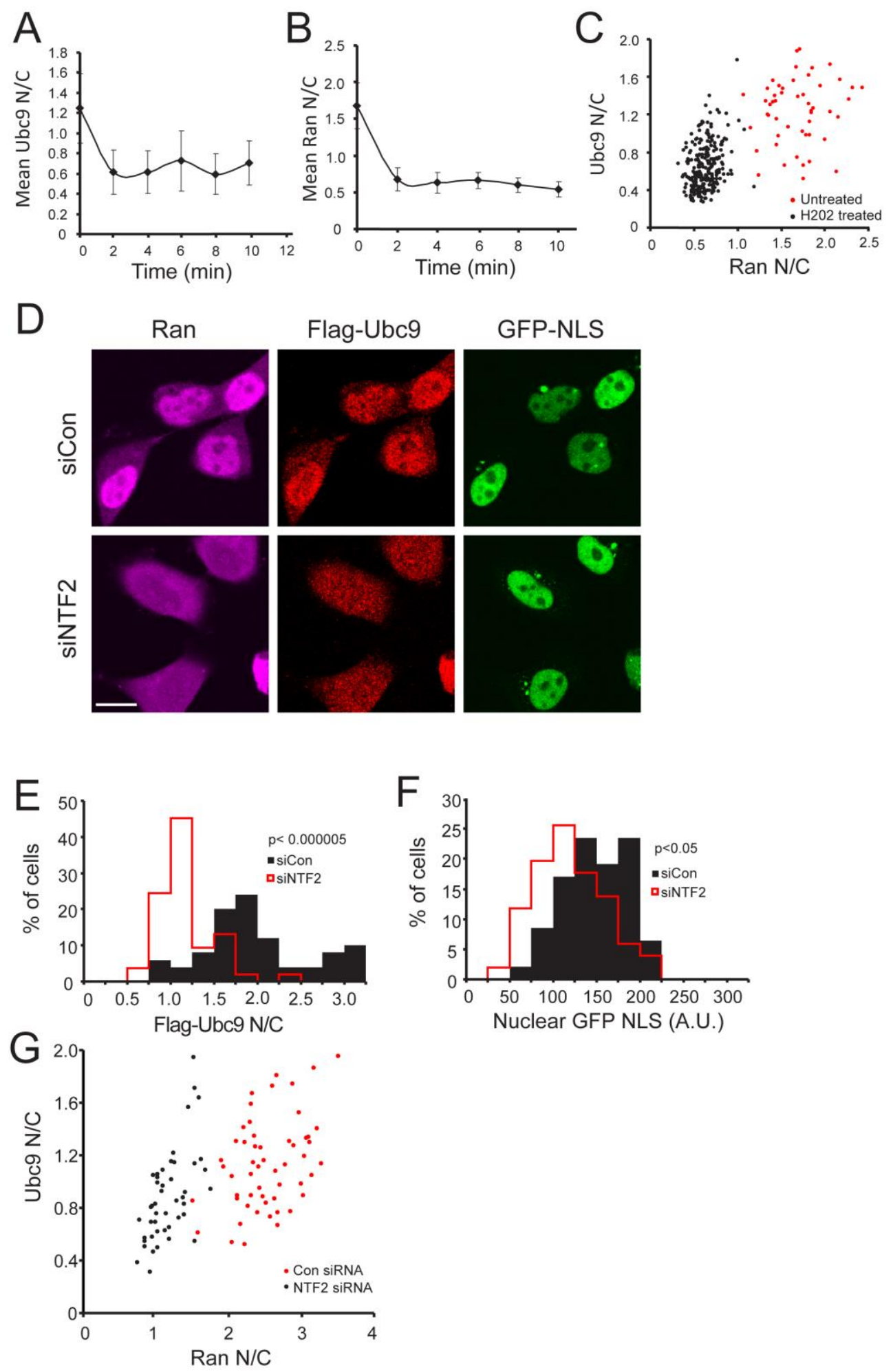
correlated with the N/C of Ran in both control siRNA and NTF2 siRNA samples, indicating that Ubc9 is dependent on the nuclear concentration of Ran for efficient nuclear localization (Fig. 19G). Thus in addition to Ubc9 mislocalization being responsible for the Ran gradient disruption in HGPS (Chapter 2), a disrupted Ran gradient can also cause defective nuclear import of Ubc9.

\section{Lopinavir, a Zmpste24 inhibitor mislocalizes Ran and Ubc9 and elevates ROS} levels.

In order to have temporal control over the induction of ROS and the Ran and Ubc9 defects, we looked for a non-transfection based method to produce the defects. Previously we had observed that inhibiting the processing of Lamin A by Lopinavir (LPV) treatment causes a loss of nuclear SUMOylation and disrupts the Ran gradient (Kelley et al., 2011). LPV is an HIV protease inhibitor that blocks the cleavage of prelamin A by Zmpste24 and as a result, WT prelamin A remains anchored to the nuclear membrane (Coffinier et al., 2007). LPV treatment has also been shown to increase cellular levels of ROS in various cell types (Capel et al., 2012; Deng et al., 2010; Lagathu et al., 2007; Lefavre et al., 2010). To check whether LPV could cause

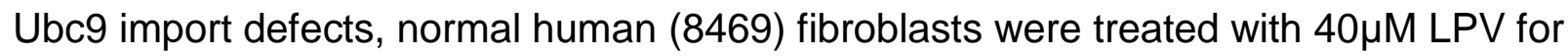
72 hours and processed for immunofluorescence detection of Ubc9 and Ran. The Ran gradient was strongly disrupted while Ubc9 was partially mislocalized from the nucleus to the cytosol with LPV treatment (Fig $20 \mathrm{~A}$ ). ROS measurements by DCF staining done in parallel, showed a significant increase with LPV treatment (Fig $20 \mathrm{~B}, \mathrm{C}$ ), thereby indicating that LPV is a suitable drug for studying the time course of Ran gradient 
Figure 20. Lopinavir, a Zmpste24 inhibitor mislocalizes Ran and Ubc9 and elevates ROS levels. (A) Endogenous Ubc9 (red) and Ran (green) distribution in normal human (8469) fibroblasts treated with DMSO (Control) or $40 \mu \mathrm{M}$ Lopinavir (LPV) for 3 days. Scale bars $=20 \mu \mathrm{m}$. (B) ROS detection in DMSO and LPV treated 8469s using DCFDA. Scale bars $=100 \mu \mathrm{m} .(C)$ Histogram of mean DCF fluorescence $($ Bin size $=10)$ measured in DMSO (Black) and LPV treated (Red) cells. 
Figure 20

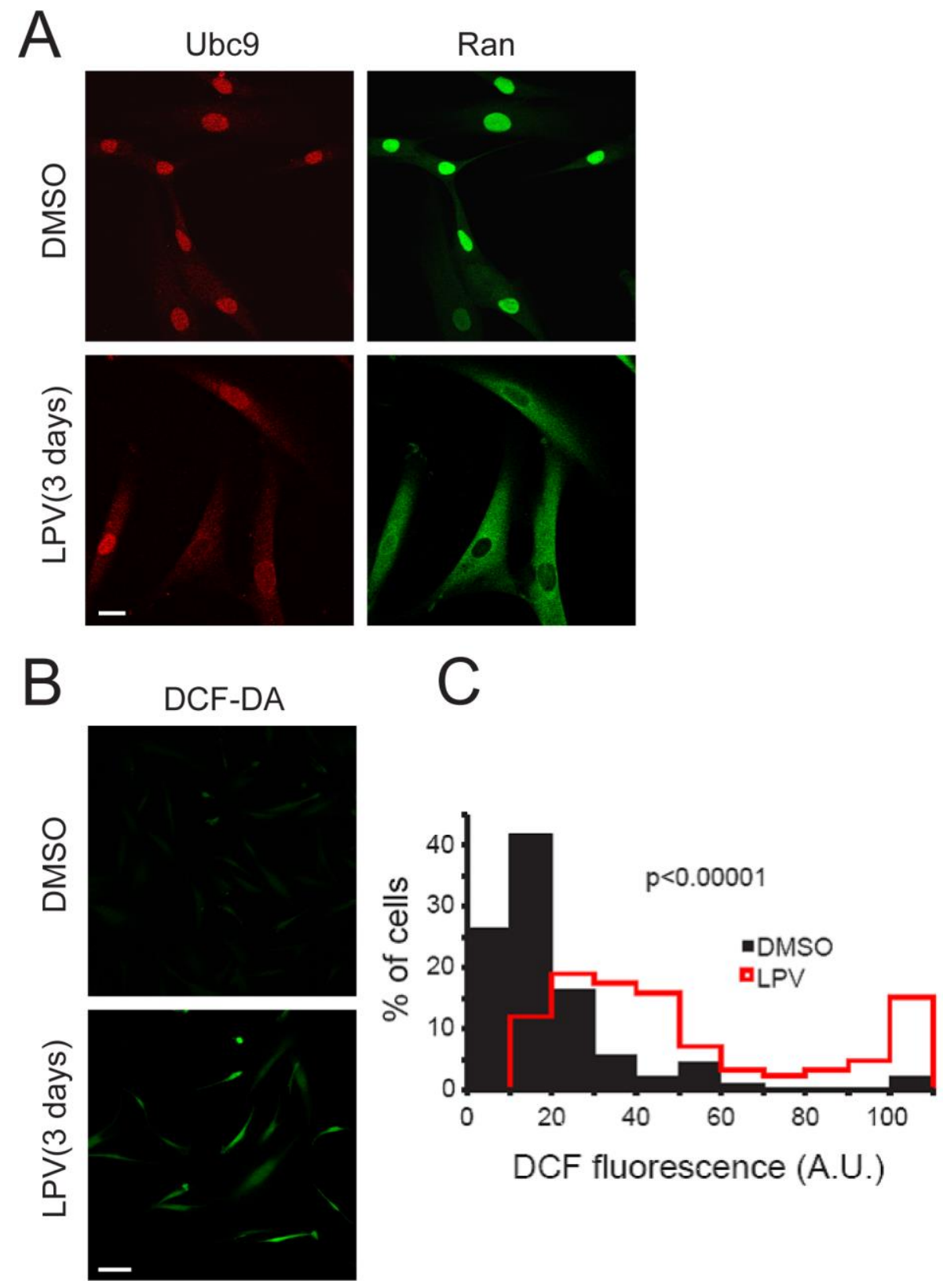


disruption and the increase in ROS levels. Owing to the partial effect on Ubc9 distribution, Ubc9 detection with LPV treatment was omitted in the subsequent experiments.

\section{siRNA knockdown of Lamin A prevents LPV induced cellular defects.}

Although LPV has been shown to inhibit Zmpste24 activity (Coffinier et al., 2007), being an aspartyl protease inhibitor, it could target other proteases in the cell leading to off target effects. Therefore in order to ask whether the effects of LPV on the Ran system and redox machinery are transduced through prelamin A, we attempted to knockdown Lamin A using siRNA, in the presence or absence of LPV treatment. Immunoblotting of prelamin A showed that knocking down Lamin A prevents the accumulation of prelamin A while the levels of Lamin A are only reduced to about half (Fig $21 \mathrm{~A}$ ). This is likely due to the fact that LPV treatment affects only newly synthesized Lamin A. Measurement of ROS levels by DCF fluorescence showed that Lamin A knock-down dramatically reduced the levels of ROS induction by LPV treatment (Fig $21 \mathrm{~B}$ ). In the same experiment, IF detection of prelamin A showed little to no prelamin A without LPV while with LPV treatment, prelamin A could be easily detected at the nuclear envelope. However upon knocking down Lamin A, prelamin A could no longer be detected, as expected (Fig $21 \mathrm{C}$ ). In the same cells, the Ran gradient was drastically disrupted with LPV treatment under control siRNA conditions but with Lamin A siRNA transfection, the Ran gradient was significantly rescued (Fig 21 C). Quantification of the Ran N/C corroborated the above findings (Fig $21 \mathrm{D})$. These 
Figure 21. siRNA knockdown of Lamin A prevents LPV induced cellular defects.

(A) Immunoblotting for prelamin A, Lamin A and Tubulin (loading control) in control and Lamin A siRNA transfected normal (8469) human fibroblasts, treated with or without LPV. (B) Bar graph depicting the mean DCF fluorescence in control and Lamin A siRNA transfected cells, with or without LPV treatment. (C) Immunofluorescence detection of prelamin A (red) and Ran (green) in control and Lamin A siRNA transfected cells, with or without LPV treatment. Scale bars $=20 \mu \mathrm{m}$. (D) Bar graph depicting the mean Ran $\mathrm{N} / \mathrm{C}$ in control and Lamin A siRNA transfected cells, with or without LPV treatment. Statistical significance as measured by student's T test is depicted by asterisks. * $p$ $<0.05,{ }^{* *} p<0.001$. 
Figure 21
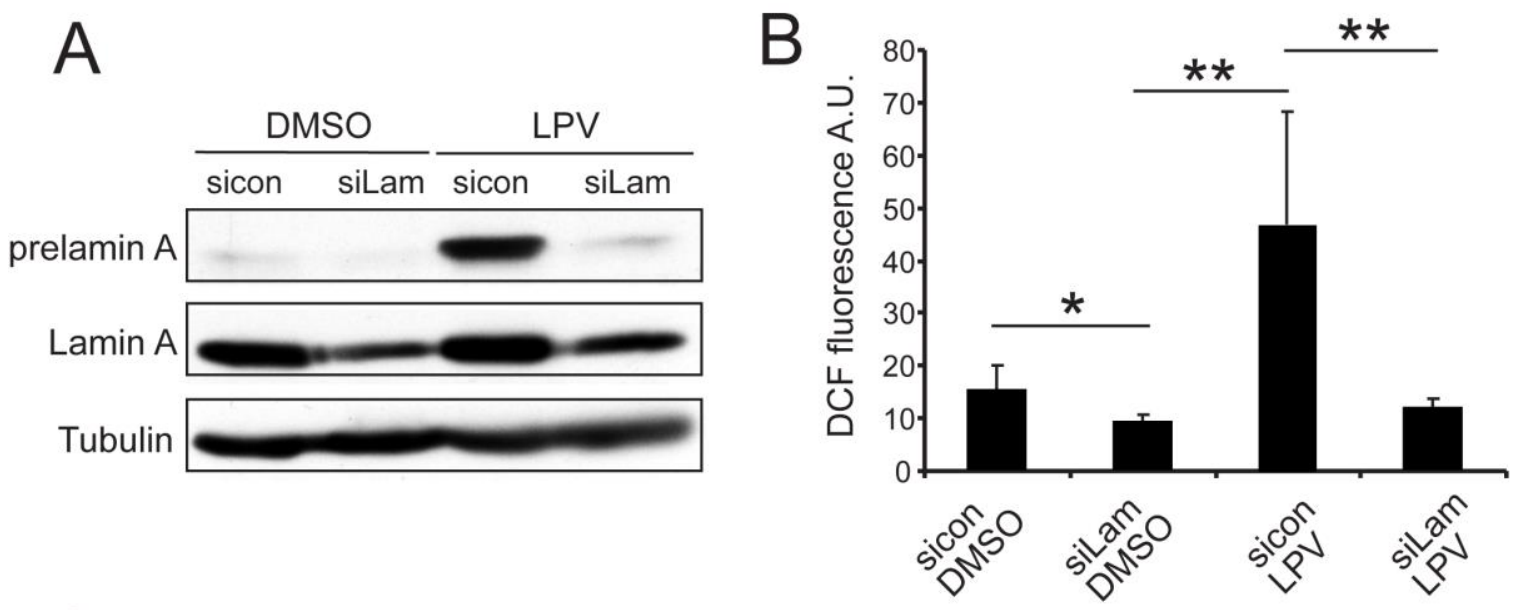

C sicon DMSO siLam DMSO
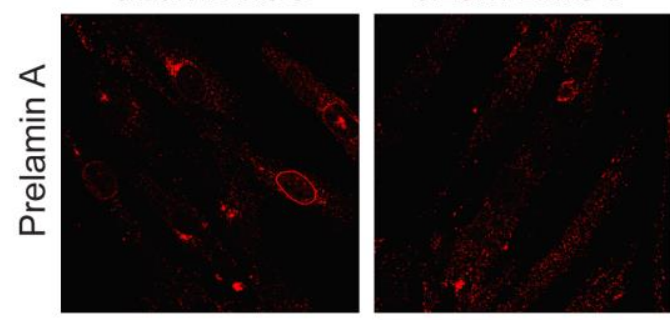

sicon LPV siLam LPV
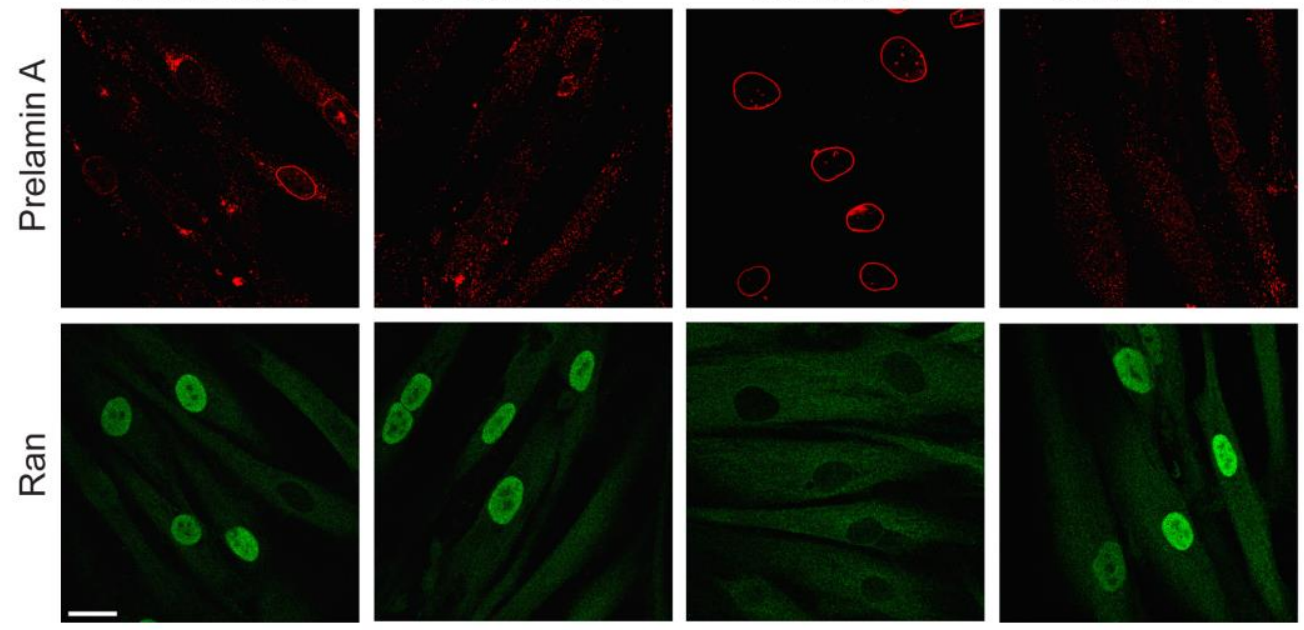

D

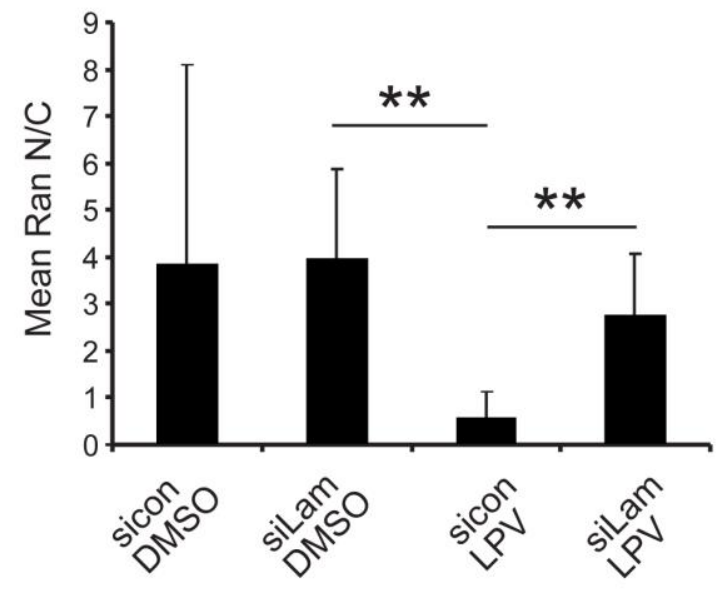


results indicate that the LPV induced cellular defects observed earlier are transduced through the nuclear lamina, thereby justifying the use of LPV to order the cellular events in HGPS, with prelamin A being a Progerin mimetic.

\section{Time course of prelamin A accumulation, disruption of the Ran gradient and induction of ROS by LPV treatment.}

A time course of LPV treatment was carried out and the amount of prelamin A induced by LPV was monitored with time by western blotting. Prelamin A was induced as early as 12 hours post treatment and maximal levels were attained by 48 hours with constant levels maintained up to 72 hours (Fig 22 A). Following washout of the drug, prelamin A levels dropped considerably, almost down to basal levels (Fig 23 A). ROS levels as measured by DCF staining showed a $\sim 1.5$ fold increase at 12 hours followed by reduction to basal levels by 18 hours. A second phase of significant ROS induction occurred at 72 hours (Fig 22 B) and this time the induction was $\sim 3$ fold. As expected its levels were restored back to basal upon drug washout (Fig 23 B). However the Ran gradient started to disrupt as early as 12 hours and by 48 hours Ran appeared to be completely cytosolic (Fig 22 C, D). Upon drug washout the Ran gradient improved although not completely (Fig $23 \mathrm{C}$ ). This implies that the defects induced by LPV treatment are not stable and can be reversed, at least partially upon washout of the drug. The increase in ROS at 12 hours could be significant or unrelated to the Ran defects, considering its low magnitude. Nevertheless, quite contrary to our expectations, the onset of Ran gradient defects preceded the second phase increase in ROS levels. 
Figure 22. Time course of prelamin A accumulation, disruption of the Ran gradient and induction of ROS by LPV treatment. (A) Immunoblot showing the accumulation of PreLamin A upon treatment of 8469 fibroblasts with $40 \mu \mathrm{M}$ LPV at the indicated time points. Ran levels remain unchanged. Tubulin represents the loading control. (B) Bar graph comparing mean DCF fluorescence at different time points of LPV treatment. Grey and Black bars represent DMSO and LPV respectively. (C) Endogenous Ran distribution (green) at the indicated time points of LPV treatment detected by immunofluorescence. Scale bars $=50 \mu \mathrm{m}$. (D) Bar graph representing the Ran N/C ratios at the indicated time points of DMSO (Grey bars) and LPV (Black bars) treatment. Statistical significance as measured by student's T test is depicted by asterisks. ${ }^{*} p<0.05,{ }^{* *} p<0.001$. 
Figure 22
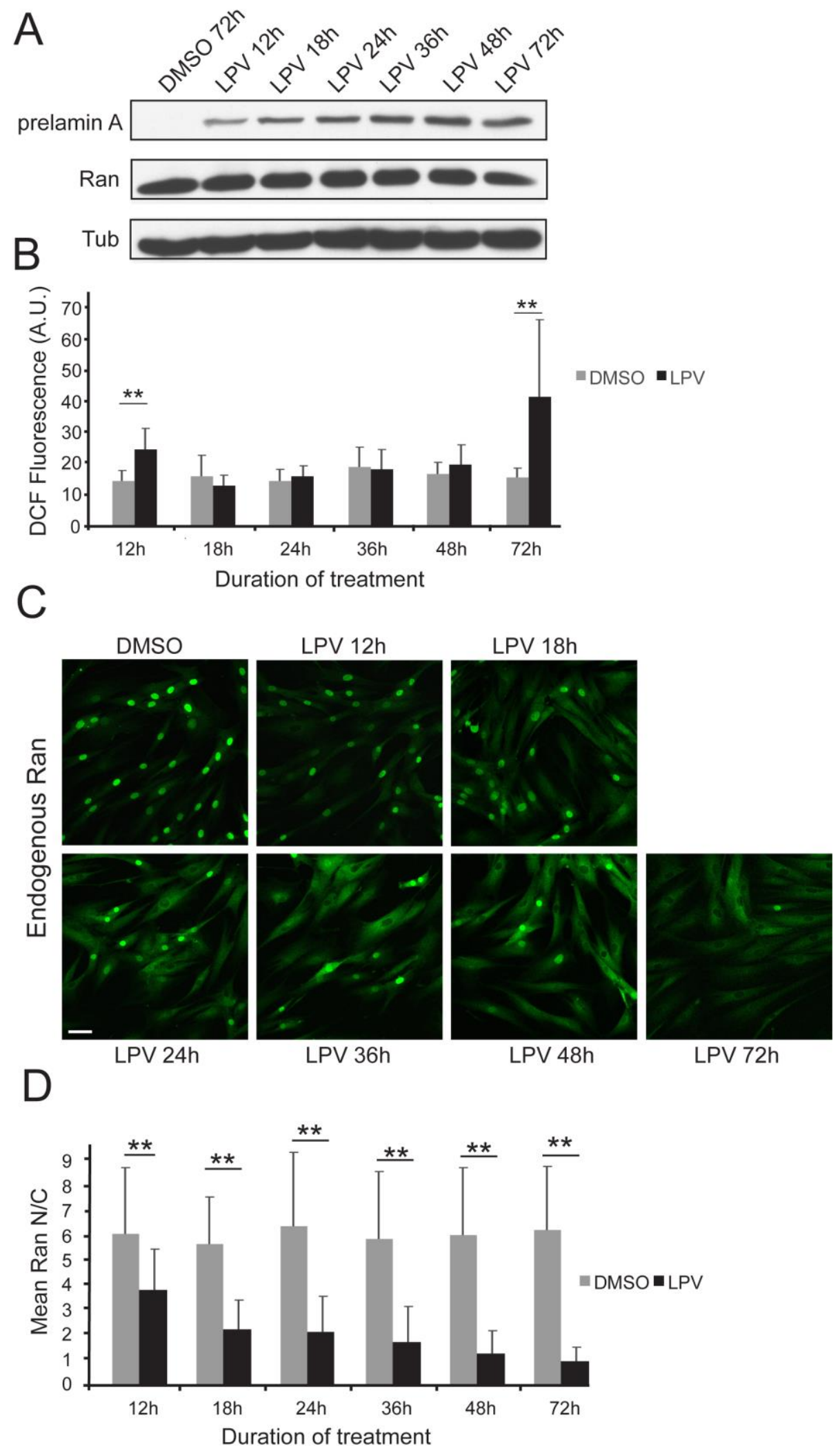
Figure 23. Induction and reversal of cellular defects with LPV treatment and post treatment withdrawal respectively. (A) Immunoblot showing the accumulation of PreLamin A upon treatment of 8469 fibroblasts with $40 \mu \mathrm{M}$ LPV and its disappearance upon drug washout at the indicated time points. Ran levels remain unchanged. Tubulin represents the loading control. (B) Bar graph comparing mean DCF fluorescence at different time points of LPV treatment and post treatment drug washout. Grey and Black bars represent DMSO and LPV respectively. (C) Bar graph representing the Ran N/C ratios at the indicated time points of LPV treatment and washout (Black bars). The grey bar shows the Ran N/C with 96 hours of DMSO treatment (control). Statistical significance as measured by student's $T$ test is depicted by asterisks. ${ }^{*} p<0.05,{ }^{* *} p<$ 0.001 . 
Figure 23

A

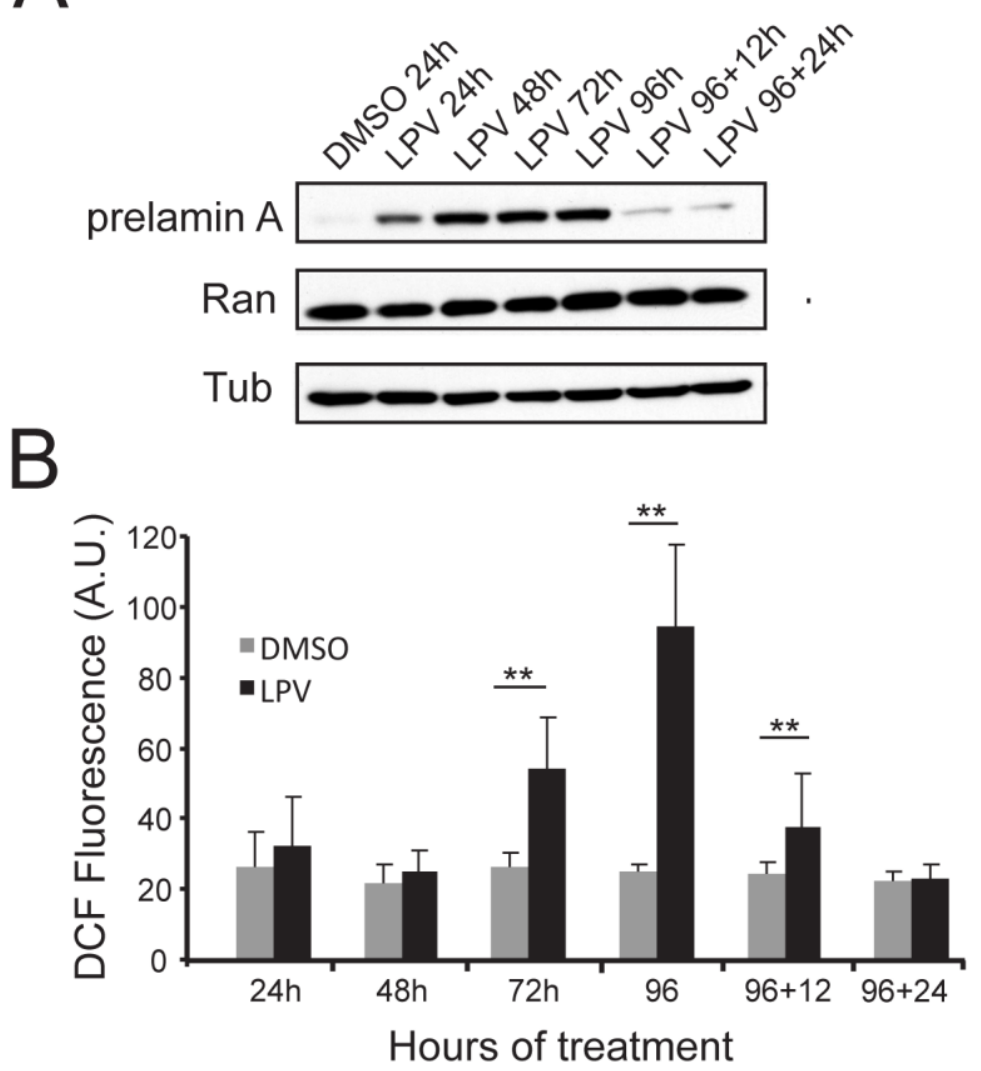

0

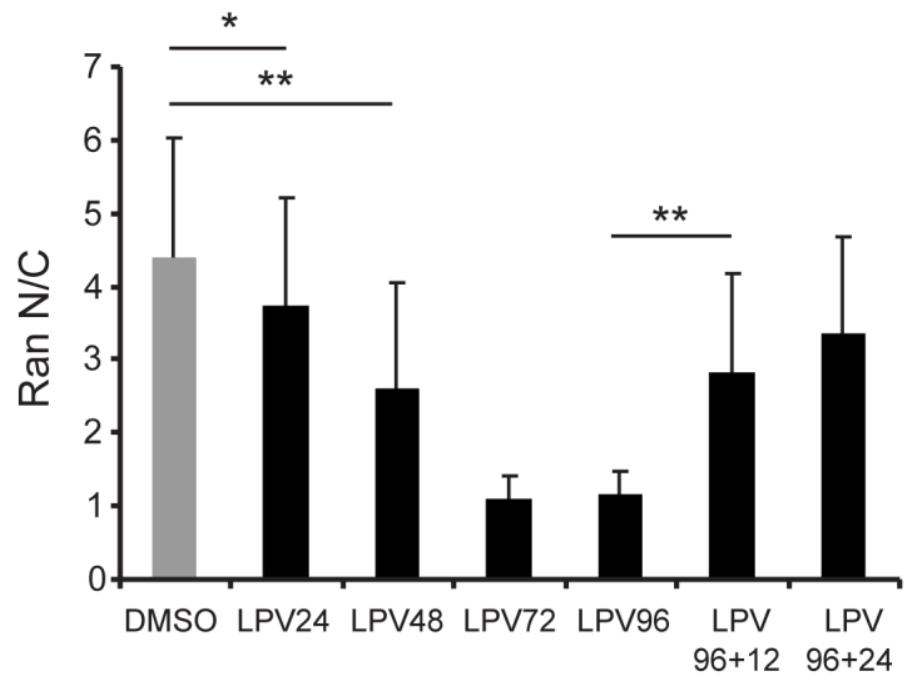

Hours of treatment 
This was surprising in light of previous reports that oxidative stress can cause Ran gradient disruption (Czubryt et al., 2000; Miyamoto et al., 2004b; Yasuda et al., 2006). The production of prelamin A however, appears to be upstream of both the Ran and ROS defects based on its early prevalence as well as the Lamin A knockdown experiments. (Figure 21). Since ATP depletion has been implicated in the disruption of the Ran gradient (Schwoebel et al., 2002; Yasuda et al., 2006), ATP levels were measured with LPV treatment and showed no significant changes while the Ran gradient was strongly disrupted (Fig $24 \mathrm{~A}, \mathrm{~B}$ ).

\section{Disruption of the Ran protein gradient is sufficient to cause an increase in cellular ROS levels.}

Taking into consideration that in the LPV treatment experiments, the Ran gradient disruption preceded the second phase increase in cellular ROS levels, we asked whether the Ran gradient could function upstream of ROS induction. To our surprise, knocking down NTF2 and disrupting the Ran gradient in normal human fibroblasts (Fig $25 \mathrm{~A}$ ) increased cellular ROS levels as measured by DCF staining (Fig $25 \mathrm{~B}, \mathrm{C}$ ), indicating that the normal nuclear levels of Ran are required for keeping cellular ROS levels under check. Thus in the context of HGPS, ROS induction may be a bi-phasic phenomenon. The initial burst of ROS could cause a disruption of the Ran gradient, which in turn can initiate the cellular changes causal to the slower secondary phase of ROS induction. 
Figure 24. ATP measurements with LPV treatment. (A) Normal human (8469) fibroblasts were treated with DMSO or LPV for the indicated time points in a 96 well plate and ATP-LITE chemiluminescence assay was used to measure ATP levels in a plate reader. The luminescence intensities were normalized to cell number and plotted as a bar graph. Grey and black bars denote DMSO and LPV treated cells respectively. The $p$ values are indicated. (B) A bar graph representing the Ran N/C with the indicated time points of LPV treatment in the same experiment. The grey bar shows the Ran N/C with 96 hours of DMSO treatment (control). Statistical significance as measured by student's T test is depicted by asterisks. ${ }^{*} p<0.05,{ }^{* *} p<0.001$. 
Figure 24

A
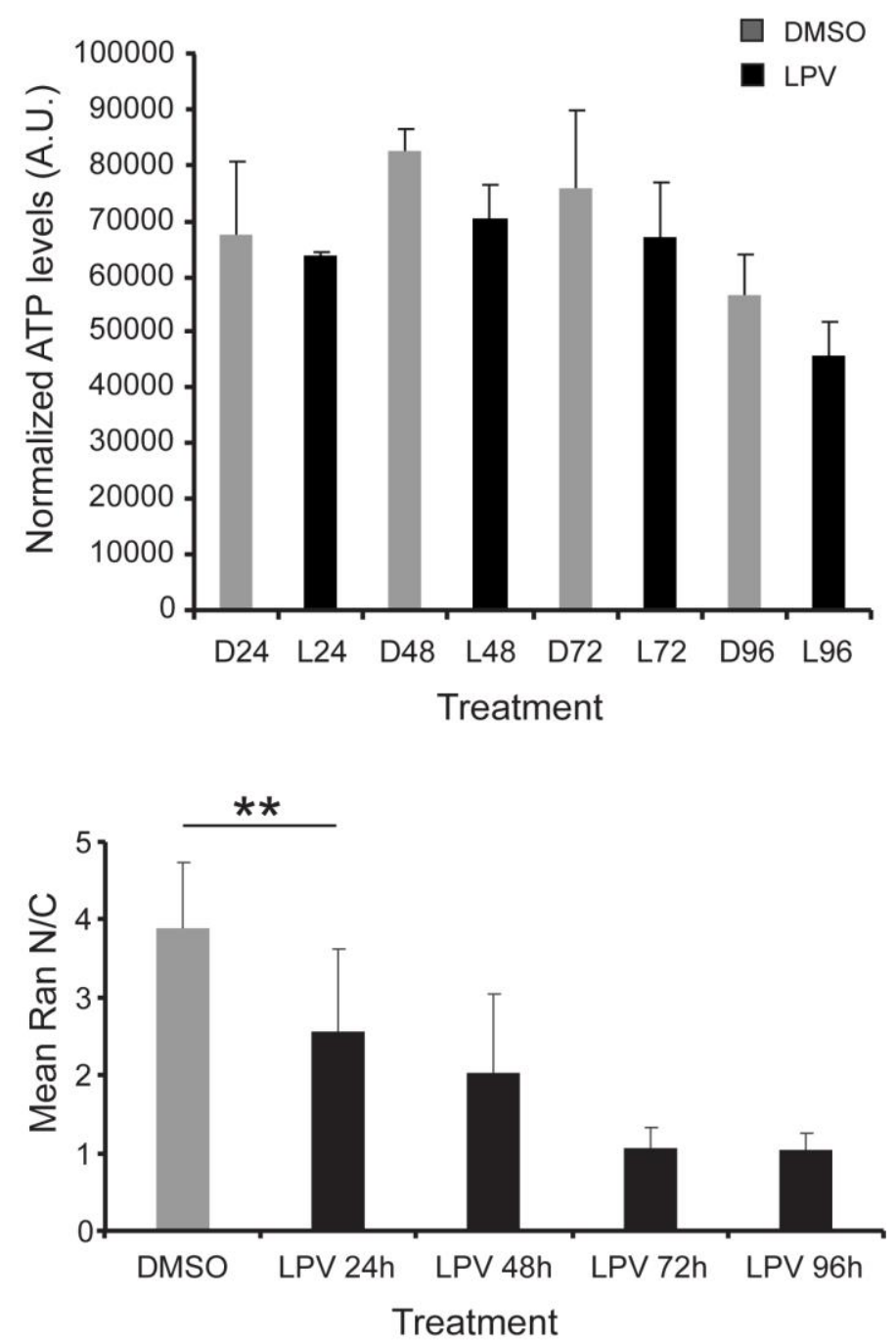
Figure 25. Disruption of the Ran protein gradient is sufficient to cause an increase in cellular ROS levels. (A) Disruption of the Ran gradient by siRNA depletion of NTF2 in 8469 fibroblasts (5 days), followed by immunofluorescence detection of Ran distribution. Scale bars $=20 \mu \mathrm{m}$. (B) ROS detection in 8469 fibroblasts treated with Control and NTF2 siRNA, showing an increase in levels upon NTF2 knockdown. Scale bars $=100$ $\mu \mathrm{m} .(\mathrm{C})$ Histogram depicting an increase in mean DCF fluorescence intensity upon NTF2 Knockdown (Red lines) in comparison to control siRNA treated cells (Black bars). Bin size $=10$. 
Figure 25

A

Ran

B

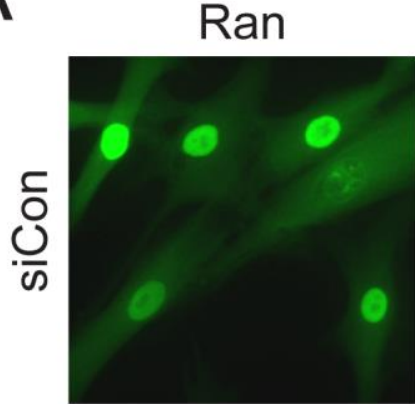

DCF-DA
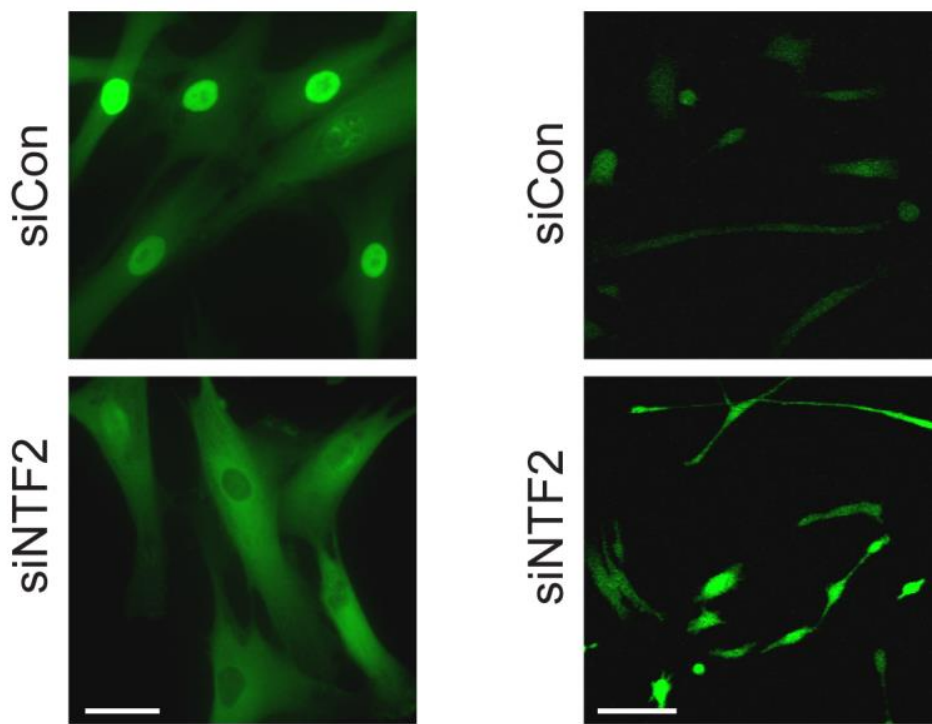

C

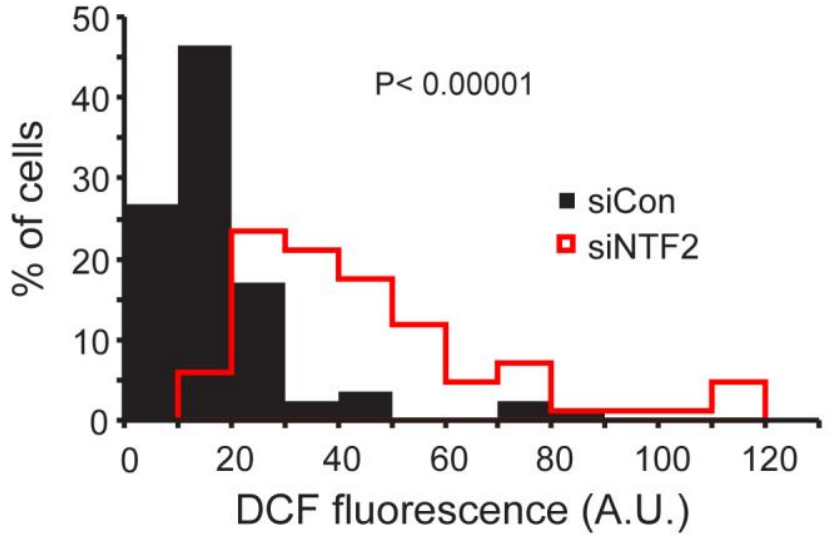




\section{DISCUSSION}

Previously we have demonstrated that the mislocalization of SUMO E2 Ubc9 from the nucleus to the cytosol in Hutchinson-Gilford Progeria syndrome, causes a global reduction in nuclear SUMOylation, which in turn disrupts the Ran protein gradient (Chapter 2). By using salt extraction methods and Fluorescence Recovery After Photobleaching (FRAP), Kelley et al. showed that the mobility of RCC1 on chromatin is regulated by SUMOylation. Since RCC1 activity is closely coupled to its on and off rate on chromatin (Li et al., 2003), Kelley et al. hypothesized that the loss of nuclear SUMOylation in HGPS disrupts the Ran gradient by affecting RCC1 activity (Kelley et al., 2011). In this study, we focused on a newly recognized property of HGPS cells, namely an increase in cellular ROS levels, and established its connection with the mislocalization of Ubc9 and Ran. Both basal as well as stimulated levels of ROS are higher in a few recently studied HGPS cell lines (Pekovic et al., 2011; Richards et al., 2010; Viteri et al., 2011). In order to establish whether this is applicable to the HGPS cell lines that we study, we measured cellular ROS levels in our HGPS cell lines using DCF staining and verified an increase in basal ROS levels, thereby indicating that oxidative stress may be a common feature of HGPS cells (Fig 12). We also showed that the mitochondria from HGPS cells are depolarized, indicative of mitochondrial dysfunction (Fig 13).

Oxidative stress has been previously shown to inhibit Ubc9 activity by inducing the formation of a disulfide bond between the catalytic cysteines of Ubc9 and the SUMO E1 Uba2 (Bossis and Melchior, 2006). In order to ask whether the localization of Ubc9 
could also be affected by oxidative stress, we treated HeLa cells with increasing concentrations of $\mathrm{H}_{2} \mathrm{O}_{2}$, which led to a mislocalization of $\mathrm{Ubc9}$ from the nucleus to the cytosol in a concentration dependent manner (Fig 14). To our knowledge, this is the first report of oxidative stress's affecting the SUMOylation machinery by altering the localization of one of the key enzymes of the pathway. $\mathrm{H}_{2} \mathrm{O}_{2}$ treatment also disrupts the Ran gradient by causing a drop in ATP levels which leads to a resulting drop in free GTP levels and subsequently affects RCC1 activity by depleting the cellular pool of RanGTP (Schwoebel et al., 2002; Yasuda et al., 2006). Defects in the localization of Nup153 as well as a reduction in the levels of Ran, Importin $\beta$ and Nup153, have also been implicated in $\mathrm{H}_{2} \mathrm{O}_{2}$ treated cells (Kodiha et al., 2004). We saw a concomitant disruption of the Ran gradient in $\mathrm{H}_{2} \mathrm{O}_{2}$ treated cells along with the Ubc9 mislocalization, and both were restored upon $\mathrm{H}_{2} \mathrm{O}_{2}$ washout, indicating that the defects are rapid and reversible (Fig 14). Thus the increase in ROS levels in HGPS could in principle, disrupt the localization of Ubc9, leading to a loss of the Ran gradient by affecting RCC1 activity, caused by reduced SUMOylation.

In order to verify the above possibility, $\mathrm{CHO}$ cells pre-adapted to Oxidative stress were treated with increasing concentrations of $\mathrm{H}_{2} \mathrm{O}_{2}$ and both Ran and Ubc9 localization were unchanged upon $\mathrm{H}_{2} \mathrm{O}_{2}$ treatment, unless at a very high concentration (Fig 15). $\mathrm{CHO}$ cells grown and maintained in the presence of $\mathrm{H}_{2} \mathrm{O}_{2}$ for multiple passages ultimately adapt to ROS by a duplication in the Catalase gene, leading to increased levels as well as activity of the Catalase enzyme (Spitz et al., 1988b). Thus these cells which can metabolize ROS, do not alter their Ran or Ubc9 distributions. To ask whether Progerin uses the same mechanism, namely an increase in ROS levels to transduce its 
effects to Ran and Ubc9, we expressed Progerin in the pre-adapted cells and both Ran and Ubc9 were indeed protected against Progerin induced disruption (Fig 16). This allowed us to posit that Progerin utilizes an increase in ROS levels to affect the distributions of Ran and Ubc9 and that when ROS levels are not allowed to increase, neither pathway is affected.

The defects in Ubc9 localization upon increased oxidative stress could be a direct effect of a disulfide formation between Ubc9 and Uba2. The small size of Ubc9 (18 $\mathrm{KDa}$ ) is expected to allow its free diffusion through the Nuclear Pore Complex, whose limit of diffusion is $\sim 40 \mathrm{KDa}$ (Mohr et al., 2009). However upon ROS stimulation, the increase in effective size of the Ubc9-Uba2 complex to $~ 110 \mathrm{KDa}$ could simply exclude Ubc9 from the nucleus. The mislocalization of Ubc9 in OC-14 (oxidative stress resistant) $\mathrm{CHO}$ cells with $800 \mu \mathrm{M} \mathrm{H}_{2} \mathrm{O}_{2}$ without a concomitant Ubc9-Uba2 disulfide formation (Fig 15) points towards the unlikelihood of this mechanism. Regardless, in order to test this directly, we made use of a mutant form of Ubc9 (C93S) which does not form the disulfide and asked whether the mutant could enter the nucleus in the face of Progerin or $\mathrm{H}_{2} \mathrm{O}_{2}$ treatment. As expected, the mutant form of $\mathrm{Ubc} 9$ was also excluded from the nucleus under both conditions. This, coupled with the fact that we could not detect the Ubc9-Uba2 disulfide in extracts from HGPS fibroblasts, indicated that the Ubc9 defects are independent of heterodimer formation (Fig 17).

Previously in our $\mathrm{H}_{2} \mathrm{O}_{2}$ treatments the disruption of the Ran gradient had been interpreted as being downstream of the Ubc9 import defects, wherein the resulting loss of nuclear SUMOylation affected RCC1 activity (Kelley et al., 2011). However in $\mathrm{H}_{2} \mathrm{O}_{2}$ timecourse experiments, time points as early as 2 minutes, showed a concomitant 
disruption of the Ran gradient and Ubc9 localization (Fig 19). We reasoned that if the Ubc9 defects were upstream of the Ran gradient disruption, then the Ran effects would be slower. However the rapidness of the Ran defects led us to hypothesize that the Ubc9 mislocalization could be a Ran dependent nuclear import defect which is expected to act in relatively shorter periods of time. Ubc9 is known to be imported into the nucleus by Importin13 (Mingot et al., 2001b). However its Ran dependence has not been documented. In order to ask if the Ran gradient is necessary for Ubc9 import into the nucleus, we knocked down NTF2 and disrupted the Ran gradient. This was sufficient to cause a mislocalization of Flag tagged Ubc9 without majorly affecting the levels of nuclear GFP streptavidin SV40 NLS (GSN) (Fig 19). This implies that the disruption of the Ran gradient is sufficient to cause a Ubc9 import defect specifically, as opposed to a general reduction in nuclear import. To this effect, Flag-NLS Ubc9 which restores the Ran gradient in the face of Progerin, also rescued the nuclear import of Myc-Ubc9 (Data not shown). Thus Ubc9 nuclear import is both upstream as well as downstream of the Ran gradient disruption and the way we envision this happening, is through a cyclic order of dependence between the two processes. In other words an intact Ran gradient is necessary for Ubc9 nuclear import that drives nuclear SUMOylation, which in turn helps maintain the Ran gradient by modulating RCC1 activity. A defect in any of these processes is expected to affect the next step and thereby cause a progressively deteriorating phenotype.

In order to pinpoint the role of oxidative stress in the development of this vicious cycle, we made use of LPV an HIV protease inhibitor that has been shown to inhibit Zmpste 24 and accumulate prelamin A which retains the farnesyl group (Coffinier et al., 
2007). The resultant protein is expected to be attached to the nuclear membrane and thereby mimic the effects of Progerin. We have previously shown that LPV treatment can disrupt the Ran gradient and reduce nuclear SUMO 2/3 levels (Kelley et al., 2011). LPV treatment has also been demonstrated to increase cellular ROS levels (Deng et al., 2010; Lagathu et al., 2007; Lefavre et al., 2010). We verified the effects of LPV on ROS levels, Ran and Ubc9 distribution and these were indeed altered in our experimental settings, although the effect on Ubc9 was moderate (Fig 20). By knocking down Lamin A in the presence of LPV treatment, we also verified that the cellular effects of LPV are indeed transduced through the nuclear lamina (Fig 21).

Having established the suitability of LPV for ordering the molecular events in HGPS, we carried out a time course of LPV treatment and monitored prelamin A accumulation, ROS levels and the Ran gradient. To our surprise, there were two phases of ROS induction. The first was at 12 hours which coincided with the initiation of the Ran gradient disruption, followed by a much later induction at 72 hours (Fig 22). To test whether the secondary induction of ROS could be downstream of the Ran gradient disruption, we disrupted the Ran gradient by knocking down NTF2 and quite unexpectedly, there was an induction in cellular ROS levels as measured by DCF fluorescence (Fig 25). Thus a temporary burst of oxidative stress could disrupt the Ran gradient while the later induction of ROS could be a slower phenomenon downstream of the Ran gradient disruption. Knocking down Ran has been shown to induce the expression of MnSOD, indicative of oxidative stress and contribute towards cellular senescence (Nagai and Yoneda, 2012).

We hereby propose a model wherein the cellular defects in HGPS can be 
subdivided into "Initiation" and "Maintenance" phases (Fig 26). Since the time-course of LPV treatment did not allow a temporal resolution of the initial burst of ROS and the onset of Ran gradient disruption, they could both be occurring simultaneously.

Therefore they both constitute the initiating events. In addition to the possibility of being a direct consequence of the initial ROS induction, the onset of disruption of the Ran gradient could be a result of perturbation of the nuclear lamina due to irreversible attachment of Progerin to the nuclear membrane. This could directly affect the structure of chromatin thereby affecting the activity of RCC1, that associates with heterochromatin and can be stimulated by binding histones H2A and H2B (Casolari et al., 2004; Nemergut et al., 2001). The resulting Ran gradient disruption could prevent the nuclear import of Ubc9, owing to which there is a loss of nuclear SUMOylation. This in turn could disrupt the Ran gradient further by modulating RCC1 activity by regulating its mobility on chromatin (Kelley et al., 2011).

The subsequent secondary induction of ROS levels by the Ran gradient disruption constitutes the "Maintenance" phase. This could be a driven by a gene expression based mechanism, specifically through altering the expression of redox scavenging enzymes, or regulators of NADPH Oxidase (NOX) complexes (Lambeth, 2004). HGPS cells have depolarized mitochondria compared to the normal fibroblasts, indicative of Mitochondrial dysfunction (Fig 13). Although Mitochondria are considered the major sources of ROS generation, by using NOX inhibitors, our lab has shown that it is possible to rescue the effects of LPV on the Ran gradient (Chelsi Snow, unpublished observations). As an additional mechanism for sustained maintenance of the Ran gradient disruption, the increase in ROS levels could potentially signal back to the Ran 
Figure 26. Model showing the proposed pathway of Progerin induced cellular defects. Under normal conditions, there exists a mutually regulatory signaling network involving the Ran GTPase system, the SUMOylation machinery and redox balance pathways. An intact Ran gradient allows the nuclear import of Ubc9 on one hand, thereby maintaining nuclear SUMOylation, which in turn regulates RCC1 activity and maintains the Ran gradient. On the other hand it also keeps ROS levels to a minimum. Progerin expression hits this network at two levels. First it disrupts the Ran-ROS balance by simultaneously affecting both. The resulting initiation of the Ran gradient disruption causes a secondary increase in ROS levels, which in turn can signal back to the Ran system for prolonged maintenance of the Ran defects. Progerin also hits the RanSUMO cycle wherein the disrupted Ran gradient inhibits the nuclear import of SUMO E2 Ubc9. The resulting loss of nuclear SUMOylation can further disrupt the Ran gradient in a feed-back loop. Thus this model highlights the central role of the Ran system in the disruption of two major cellular pathways namely redox balance and nuclear SUMOylation and lays out the initial events originated in the nuclear lamina that could lead to HGPS progression at the cellular level. 
Figure 26

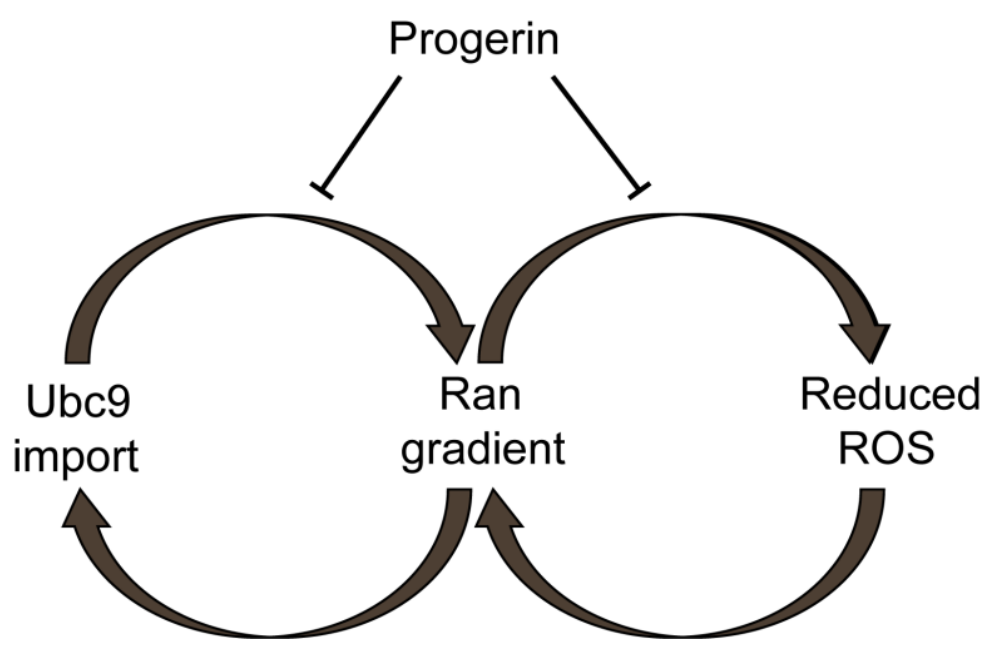


system and further disrupt the Ran gradient, most likely by modifying Redox sensitive cysteines on RCC1 and thereby lowering its activity (Mandovi Chatterjee, unpublished observations). Previously published mechanisms involving the lowering of RanGTP levels are not likely to be involved because LPV treatment did not cause a reduction in cellular ATP levels in our hands, while the Ran gradient was disrupted (Fig 24). The fact that $\mathrm{CHO}$ cells pre-adapted to Oxidative stress do not have Ran or Ubc9 localization defects, can easily be explained by the above model wherein Progerin expression does probably induce an initial disruption of the Ran gradient and Ubc9 mislocalization, but since the resulting secondary phase ROS induction is not allowed to take place, the secondary maintenance phase of the pathway is not triggered. As a result the initial loss of the Ran gradient could be corrected by the end of 24 hours.

Thus in this study, our observations linking the disruption of the Ran gradient, the loss of Ubc9 nuclear import and the induction of cellular ROS levels indicate the presence of a complex signaling network that when disrupted, is expected to compromise normal nuclear functioning. This network represents the early events that follow the perturbation of the nuclear lamina and could potentially precede an alteration of global gene expression patterns within the nucleus. The culmination of aberrant signaling cascades and disrupted gene expression programs may subsequently contribute to premature aging at the cellular level. 


\section{Chapter 4. CONCLUSIONS AND FUTURE DIRECTIONS.}

Ever since the discovery that HGPS is caused by a mutation in the Lamin A gene, a plethora of observations have been made that document the features of the disease at the cellular level. These observations however are mostly restricted to events that are either upstream, for example the perturbation of the nuclear lamina or are mostly downstream phenotypes like the alteration in gene expression patterns. Considering the huge separation between these events, the aim of this thesis has been to bridge the gaps and provide mechanistic insights into intermediate pathways that connect the disruption of the nuclear lamina with global changes in gene expression. In this work, we have focused on nuclear SUMOylation, the Ran gradient and ROS levels, three pathways fundamental to normal cellular functions, which potentially provide previously unappreciated connections between the very upstream and downstream defects in HGPS. In addition to reporting that these pathways are defective in HGPS, we provide evidence for the existence of a complex signaling network involving each of these components that is highly sensitive to perturbation. The misregulation of these pathways in HGPS is likely to result in a global disruption of cellular functions. Thus a study of these pathways facilitates an understanding of the cellular progression of HGPS. However an attempt towards understanding these pathways raises many important questions.

Our founding observation was the disruption of the Ran protein gradient by reducing nuclear SUMOylation. Our use of SENP CDs to disrupt nuclear SUMOylation 
raises a question about the localization of the SENPs themselves in the context of HGPS. In addition to the Ubc9 import defect, one or more of the SENPs may be mislocalized as a supporting mechanism to result in a reduced SUMOylation status in the nucleus. This possibility warrants a systematic approach towards characterizing SENP localization defects in HGPS patient cells and with Progerin expression. Notably, we have observed a striking mislocalization of SENP1 from the nucleus to the cytoplasm with the expression of HA-Progerin (Our unpublished observations). Arguably this should serve to increase nuclear SUMOylation and reduce cytosolic SUMOylation. However a complete picture of the localization status of all the SENPs is necessary before stating a conclusion regarding the role of one or more of the SENPs in the reduction of nuclear SUMOylation. If the mislocalization of SENPs has a major role in HGPS, then siRNA knockdown of the SENPs either singly or in combination in the context of Progerin expression, may rescue the Ran gradient by causing a net increase in nuclear SUMOylation.

One of the striking phenotypes that led us to be interested in the in-depth analysis of nuclear SUMOylation defects in HGPS, was the mislocalization of Ubc9. We demonstrate that this is due to a disruption of the Ran gradient thereby leading to an import defect. Ubc9 is imported into the nucleus by Importin13 an importin $\beta$ family member (Mingot et al., 2001a). Although we have verified that the localization of Importin 13 is unchanged when Ubc9 is mislocalized (our unpublished observations), a detailed characterization of the import defects is necessary. Despite the incomplete characterization of the Ubc9 import pathway, one may speculate that it conforms to the normal importin $\beta$ dependent import pathways. Thus the pathway of Ubc9 nuclear 
import is expected to involve a complex formation with Importin13, translocation of the complex through the Nuclear Pore complex(NPC) followed by dissociation of the complex by RanGTP binding. The Ubc9 import defect in HGPS may involve abnormalities at any of these steps. It would be useful to test the binding of Ubc9 to Importin13 and also compare the concentration of RanGTP required to dissociate the complex, under normal and Progerin expression conditions. In a recent study, the authors have laid out differences between the RanGTP requirements of large vs small cargoes (Snow et al., 2013). Although Ubc9 itself is a small protein, the complete biochemical composition of its import complex is unknown. It is formally possible that the import complex of Ubc9 with Importin13 has additional partners that contribute towards the formation of a large complex, which has stringent RanGTP requirements for dissociation. In our hands, Ubc9 import defects only occur when the Ran gradient is strongly disrupted. Thus it may be essential to use chromatographic techniques to figure out the actual size of the Ubc9 import complex and identify unknown partners that may impose another layer of regulation.

Restoring the nuclear localization of WT Ubc9 in the context of Progerin rescues the Ran gradient. Since the Ran gradient strictly depends on RCC1 (Ren et al., 1993; Tachibana et al., 1994), we speculate that this is due to stimulation of RCC1 activity in the nucleus. Our speculation is backed by the finding that decreasing nuclear SUMOylation reduces the mobility of RCC1 on chromatin and in vitro SUMOylation of an yet unidentified target on chromatin promotes the release of RCC1 from chromatin (Kelley et al.). Since RCC1 activity is closely coupled to its on and off rate on chromatin (Casolari et al., 2004; Li et al., 2003; Nemergut et al., 2001; Wong et al., 2008), NLS- 
Ubc9 may simply improve RCC1 mobility and thus stimulate its activity. Particularly worth mentioning is the fact that only active Ubc9 is capable of rescuing the Ran gradient while a catalytically inactive mutant of Ubc9 fails to do so. This agrees with our hypothesis that an actual SUMOylation event by Ubc9 is necessary for the maximal dissociation of RCC1, as opposed to a non-covalent SUMO binding interaction by a SUMO interaction motif (SIM) on the target protein (Kelley et al., 2011). Although histones that stimulate RCC1 activity have been shown to be SUMOylated (Nemergut et al., 2001; Shiio and Eisenman, 2003), additional targets of SUMOylation may be involved. Thus it would be worth identifying SUMO targets that are particularly relevant to the disruption of the Ran gradient in HGPS and in general, that would provide insights into additional SUMO based mechanisms for exacerbating the cellular defects in HGPS.

In addition to rescuing nuclear SUMOylation and the Ran gradient, the restoration of Ubc9 nuclear import in the context of Progerin expression also rescued the import of nucleoporin TPR and increased the levels of H3K9 trimethylation in the nucleus. This led us to ask if in the absence of Progerin these pathways were dependent on nuclear Ubc9 levels. However to our surprise knocking down Ubc9 did not affect the Ran gradient nor did it reduce the nuclear enrichment of TPR and H3K9me3. We speculate this as being the result of a differential degree of disruption of SUMOylation between Ubc9 knockdown and Progerin expression conditions. While progerin expression dramatically reduces SUMOylation in the nucleus, a complete Ubc9 knockdown only partially equilibrates SUMO between the nucleus and the cytoplasm (Our unpublished observations). It goes without saying that Ubc9 knockdown and Progerin expression 
present completely different scenarios with respect to Ubc9, namely the latter is expected to increase Ubc9 in the cytoplasm. Interestingly the artificial targeting of Ubc9 to the cytosol (Ubc9-NES) does not affect cellular SUMOylation or the Ran gradient (our unpublished observations). In fact, quite contrary to our expectations, knocking down Ubc9 actually partially protected the Ran gradient against disruption by Progerin and by oxidative stress (our unpublished observations). Thus it is formally possible that Ubc9 acts as an inducer of the response and being a rapidly shuttling protein (Our unpublished observations), its shuttling function may be coupled to its role in the induction of the response. In other words the scenario with respect to Ubc9 may be complicated in HGPS and further characterization of these fine-tuned mechanisms is necessary.

In addition to SUMOylation machinery and Ran system defects, we and others demonstrate the existence of oxidative stress in HGPS (Viteri et al., 2011) (Richards et al., 2010) (Pekovic et al., 2011). A byproduct of normal metabolism, ROS play a significant role in normal aging most prominently through DNA damage, cellular senescence as well as increasing nuclear leakiness by oxidative damage to nucleoporins (Beckman and Ames, 1998; D'Angelo et al., 2009; Hagen et al., 1997; Sedelnikova et al., 2010). Being a premature aging syndrome, HGPS is expected to involve similar mechanisms, at least partially. In the process of characterizing the interplay between oxidative stress and the Ran and SUMOylation systems, we came across a complex array of feed-back loops that allow mutually regulatory mechanisms to work between the three parameters. Based on the evidence presented in this work we propose that the Ran gradient disruption and the initial burst of ROS induction 
appear to occur simultaneously. The initial burst of ROS could be directly caused by damage to the mitochondria by aggregates of Progerin farnesylated in the cytosol by the ER resident farnesyl transferases. WT Lamin A has an NLS between the alpha helical coiled coil domain and the Ig fold domain (Dechat 2008). Since only the C terminus of Lamin A is affected by the HGPS mutation, Progerin should have an intact NLS and therefore is capable of undergoing nuclear import. However since farnesylation of Progerin can occur in the cytoplasm, the resulting product should have a high affinity for membranes (Barrowman et al., 2008). The non-specific nature of this affinity could allow Progerin to indiscriminately attach to the ER, Golgi or Mitochondrial membranes even before it can be bound and transported into the nucleus by the nuclear import machinery, and in the process, damage mitochondria or affect ER resident NOX activity. This can be tested by fractionating mitochondrial and ER membranes from HGPS cells and checking for the enrichment of Progerin. Additionally a mutant form of Progerin lacking a functional NLS or with a mitochondrial targeting sequence may be overespressed in cells to check if it targets to the mitochondrial membranes and induces ROS. These experiments would provide fundamental insights into the possible mechanism of early induction of ROS by Progerin.

The Ran gradient disruption could potentially be a downstream consequence of this initial burst of ROS. It may also be attractive to speculate the Ran gradient disruption as being a direct effect of perturbation of the lamina by Progerin expression, altering chromatin structure by disrupting the lamina-chromatin interface and thereby affecting RCC1 activity. Indeed ING1 and LAP2, proteins that bridge the lamina with chromatin, are mislocalized in HGPS (Han et al., 2008; Scaffidi and Misteli, 2005b; 
Scaffidi and Misteli, 2006). Since Ubc9 is a rapidly shuttling protein, it is expected to be rapidly mislocalized from the nucleus to the cytosol in response to the Ran gradient disruption (Fig 27). The resulting loss of nuclear SUMOylation may help stabilize the Ran gradient defects because SUMOylation of a yet unknown chromatin associated factor can stimulate $\mathrm{RCC} 1$ release from chromatin and thereby enhance its activity (Kelley et al., 2011). RCC1 activity has been previously shown to be regulated by kinase signaling cascades through phosphorylation of RanBP3, a co-factor for RCC1 enzymatic activity (Yoon et al., 2008). Owing to a drastic loss of heterochromatin in HGPS (Shumaker et al., 2006a), RCC1 may be in a state of heavy dependence on these stimulatory pathways. Due to loss of nuclear SUMOylation, RCC1 could potentially lose this additional wing of stimulation, leading to a gradual loss of the Ran gradient. The net result of this cycle of regulatory events would be a stabilization of the Ran gradient defects.

Once the Ran gradient defect is stabilized, the secondary phase of the pathway is triggered. This includes the secondary induction of ROS which according to our findings is a much later phenomenon and by that time the Ran gradient is completely reversed. While many Ran dependent cargoes can still undergo import under conditions of moderate Ran gradient disruption, under these severe disruption conditions, nuclear import may be stalled. Some of the affected cargoes could be transcription factors that regulate redox balance in the cell. For example the FOXO proteins (Forkhead group of transcription factors) undergo nuclear import under conditions of oxidative stress and turn on the expression of MnSOD and Catalase (Alcendor et al., 2007; Brunet et al., 2004; Kops et al., 2002; Nemoto and Finkel, 2002; Van der Heide et al., 2004). The 
Figure 27. Model depicting the interdependence of nuclear SUMOylation and the Ran gradient and how it is disrupted in HGPS. Under normal conditions (right), RCC1 that associates with heterochromatin generates the RanGTP gradient which dissociates Ubc9-Importin13 import complexes, thereby releasing Ubc9 in the nucleus. Ubc9 can SUMOylate a yet unknown protein ' $x$ ' that when SUMOylated can facilitate the release of RCC1 from chromatin. This would enhance RCC1 activity which is closely coupled to its on and off rate on chromatin. Thus both nuclear SUMOylation and the Ran gradient are maintained. In HGPS (left), the expression of Progerin disrupts the nuclear lamina and alters the structure of chromatin such that it is in a more open conformation. This could affect RCC1 activity leading to disruption of the Ran gradient, in the absence of which, import complexes cannot be dissociated. As a result the Importin13-Ubc9 complex could shuttle back into the cytoplasm, leading to Ubc9 import defects. Due to its absence from the nucleus, Ubc9 cannot SUMOylate $x$ and RCC1 loses the stimulatory input from $\mathrm{x}$. Thus nuclear SUMOylation is reduced and the Ran gradient is disrupted. 
Figure 27

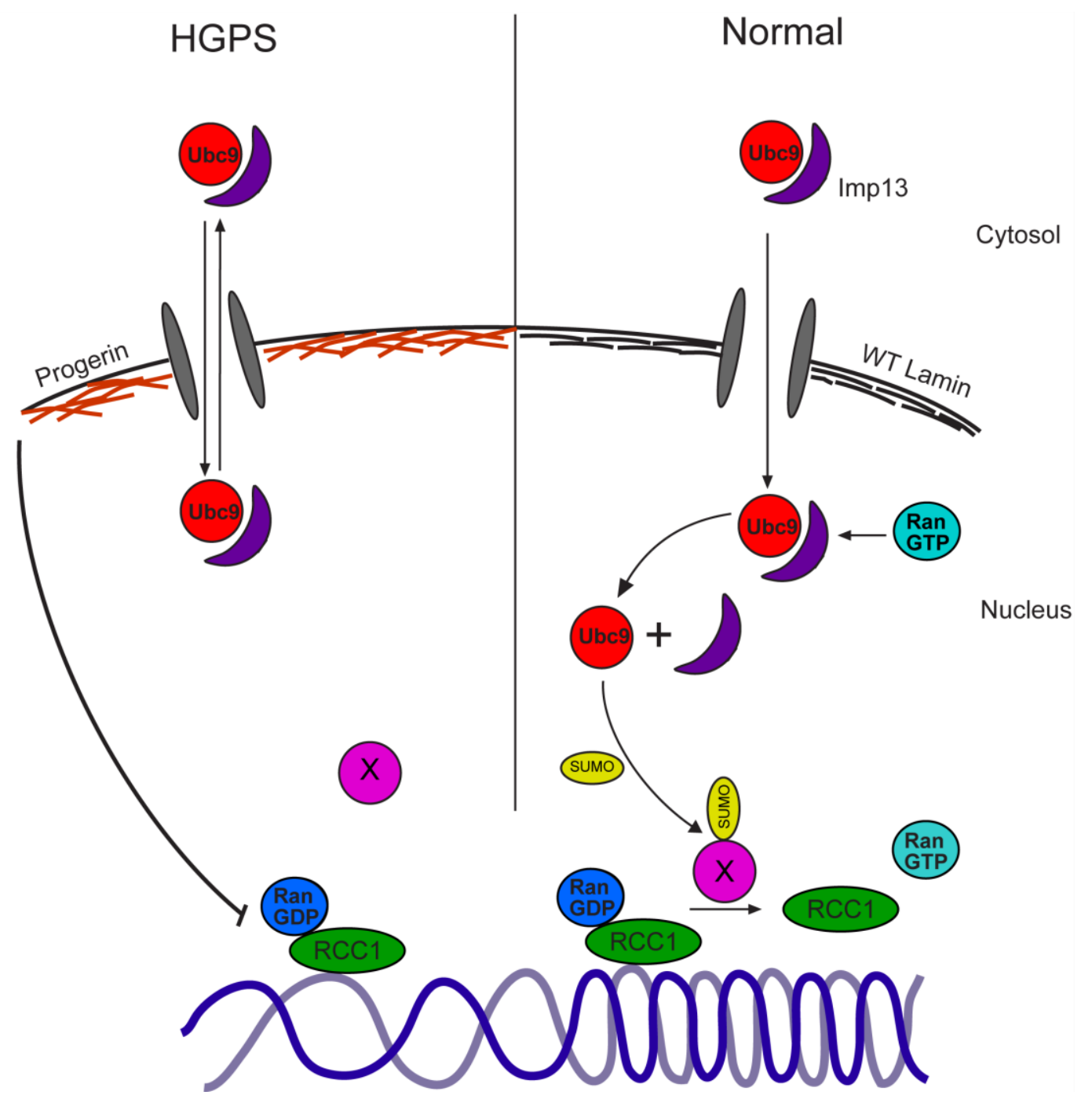


absence of an intact Ran gradient may prevent them from entering the nucleus and turning on pathways that would normally keep basal levels of ROS from increasing. This would lead to a gradual increase in ROS levels (Fig 28). Interestingly Lamin B is mislocalized in HGPS cells (Scaffidi and Misteli, 2005a) and Lamin B is responsible for tethering the transcription factor Oct-1 and keeping it inactive. In the absence of this tethering, Oct-1 becomes active and upon entering the nucleus stimulates ROS by repressing antioxidant genes (Malhas et al., 2009). Furthermore in cells aged in culture, Oct-1 dissociates from the Lamina and turns on the expression of cellular aging associated genes (Imai et al., 1997). However the likelihood of the Lamin B-Oct-1 pathway is less owing to the complete reversal of the Ran gradient. Unless the Ran gradient requirements for Oct-1 nuclear import are not stringent, the reversed Ran gradient should prevent Oct-1 from entering the nucleus and exerting its effects. Currently our hypothesis that the Ran gradient disruption is upstream of ROS induction in HGPS is backed by two observations, namely the appearance of Ran defects prior to increased ROS in the time course of LPV treatment, and ROS induction by disruption of the Ran gradient upon knocking down NTF2. It may be important to follow up these experiments with the use the Flag-NLS-Ubc9 to rescue the Ran gradient and prevent ROS induction in Progerin transfected or LPV treated cells. Microinjection of Ran into the nucleus may also be attempted to see if it brings down ROS levels in LPV treated cells. Surprisingly in the LPV timecourse experiments, the secondary induction of ROS was not gradual. Rather the levels of ROS remained constant up to 48 hours followed by a several fold increase at 72 hours. This implies that there are additional extremely slow means of misregulation of the cellular redox balance, some that take longer to 
Figure 28. Model demonstrating the possible ways by which Progerin could induce ROS. Mitochondria continuously produce basal levels of ROS which participate in cellular homeostasis, by acting as a component of signaling pathways. Under normal conditions, in response to ROS generation, the FOXO group of transcription factors enter the nucleus in a RanGTP dependent manner and enhance the expression of redox sensitive genes like MnSOD and Catalase. These enzymes keep ROS levels under check. During HGPS, the disruption of the Ran gradient by Progerin expression may inhibit the nuclear import of FOXO, thereby preventing the overexpression of the Catalase and MnSOD genes. As a result ROS levels may continue to increase. In addition, the aggregate of Progerin at the nuclear membrane could potentially induce the Unfolded Protein Response (UPR) in the lumen of the ER. The resulting leakage of Calcium ions from the ER stores could alter the permeability of the mitochondrial membranes, leading to dissipation of the mitochondrial proton gradient and generation of ROS. 
Figure 28

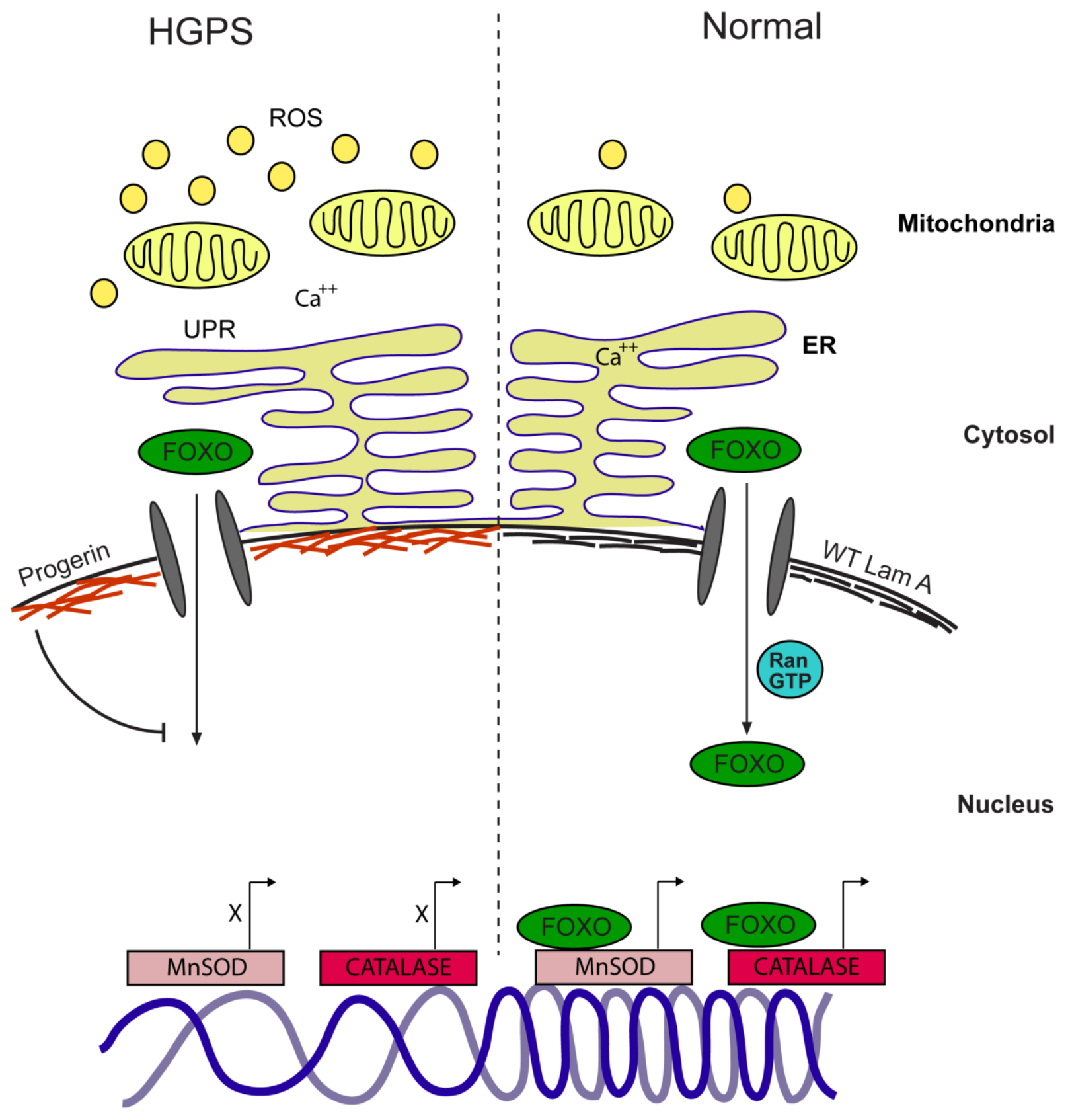


develop than gene expression based mechanisms. Since the Ran gradient is disrupted much earlier, these slow mechanisms presumably do not involve the Ran gradient disruption. We observed a depolarization of mitochondria in HGPS cells, indicative of mitochondrial dysfunction. How then could mitochondria be affected by a signal emanating from the nuclear lamina? The possibilities are fascinating (Figure 28). Since Progerin retains its farnesylation at its C terminus (De Sandre-Giovannoli et al., 2003; Eriksson et al., 2003a), it could lead to the formation of insoluble aggregates at the nuclear membrane. Since the ER lumen is continuous with the perinuclear space, the aggregate of Progerin at the nuclear membrane can be perceived as misfolded protein, leading to an Unfolded Protein Response (UPR) in the ER (Shang, 2005). ER stress has been linked with oxidative stress in yeast as well as C. elegans (Harding et al., 2003; Haynes et al., 2004). One of the end results of UPR is the leakage of Calcium ions from their stores in the ER, to the cytosol, thus increasing cytosolic Calcium concentrations (Deniaud, 2007). Furthermore, an increase in cytosolic $\mathrm{Ca}^{2+}$ has been implicated in permeabilizing mitochondrial membranes, dissipating the proton gradient and increasing oxidative stress (Deniaud, 2007; Malhotra and Kaufman, 2007; Tan et al., 1998). Although this mechanism seems far-fetched prima-facie, it serves as an interesting platform for investigation and provides a potential explanation for why the secondary ROS induction takes so long to develop despite the non-gradual nature of the induction. Testing for evidences supporting each step of the above pathway could be crucial towards understanding a novel mechanism of regulation of oxidative balance by the nuclear lamina.

Artificial induction of oxidative stress by $\mathrm{H}_{2} \mathrm{O}_{2}$ treatment is capable of disrupting 
the Ran gradient and causing a rapid and reversible mislocalization of Ubc9 from the nucleus to the cytoplasm. This led us to posit that the ROS induction in HGPS is upstream of the Ran and Ubc9 defects. However such an assumption demands caution. $\mathrm{H}_{2} \mathrm{O}_{2}$ treatment is a rather harsh treatment for cells and the concentrations of $\mathrm{H}_{2} \mathrm{O}_{2}$ that affect Ran and Ubc9 distribution are much higher than physiological, and presumably exceed the concentration of ROS produced in HGPS. It would be worthwhile to calculate the exact concentration of ROS produced in HGPS cells or with LPV treatment. In addition to the above, the use of $\mathrm{CHO}$ cells adapted to oxidative stress presents certain disadvantages. Although the adaptive response to chronic oxidative stress results in Catalase gene duplication and increased Catalase activity (Hunt et al., 1998), that represents only one of the plethora of adaptive modifications that the $\mathrm{CHO}$ cells may undergo. Therefore one must be careful with the assumption that it's because of the ability to metabolize ROS that the resistant $\mathrm{CHO}$ cells have an intact Ran gradient with Progerin expression. It would be interesting to knockdown or inhibit Catalase in the resistant $\mathrm{CHO}$ cells and ask if they can still protect their Ran gradient from Progerin or $\mathrm{H}_{2} \mathrm{O}_{2}$. Thus it is safe to conclude that while increased ROS is capable of altering the distribution of Ran and Ubc9 in ways that match HGPS related events, in the actual context of HGPS, this may be an open possibility. Our attempts to answer this question were only met with moderate success as redox scavengers like $\mathrm{N}$-acetyl Cysteine (NAC) and Catalase could only result in a partial rescue of phenotypes and was also confounded by variability on an experiment to experiment basis. We hypothesize this as being the result of the inability of redox scavengers to reduce ROS levels down to the level that would allow successful rescue of the phenotypes, due to the overwhelming 
nature of Progerin in HGPS cells or prelamin A induced by LPV treatment. As per our observations, redox scavengers do not reduce the levels of prelamin A in LPV treatment experiments. On the other hand in LPV timecourse experiments, a wash out of the drug brings down prelamin A to basal levels and therefore allows the Ran gradient to be reestablished and ROS levels to be reduced. Further trials may reveal a suitable redox scavenger that can bring down ROS levels and rescue some of the cellular phenotypes associated with HGPS.

Since the aim of this work was to bridge the gap between the upstream events in HGPS, namely lamina perturbation and the downstream consequences, it would be useful to discuss how this work can influence the understanding of the latter. The downstream phenotypes that interest us are DNA damage and gene expression changes. Oxidative stress in HGPS has already been shown to be upstream of DNA damage and cell cycle arrest (Richards et al., 2011). Once we identify a suitable redox scavenger that can successfully reduce ROS levels in HGPS cells, it would be worthwhile to see if it restores the expression patterns of a subset of genes misregulated in HGPS. Genes related to ECM function (MMP3, HAS3, TIMP3) have been shown to be downregulated in HGPS fibroblasts while those involved in an inflammatory response (CCL8, MCP-1) and stem cell proliferation (MEOX2) are overexpressed (Scaffidi and Misteli, 2005; Csoka et al., 2004). A restoration of normal expression patterns of the above genes for example, in HGPS fibroblasts with scavenger treatment, would imply that these are regulated by oxidative stress. The same genes could be studied in the context of SENP2 CD expression (reduction of nuclear SUMOylation) and NTF2 Knockdown (disruption of the Ran gradient) to check if 
nuclear SUMOylation and nuclear import-export pathways need to be functional for the normal expression patterns of these genes. If the above results hold true, then NLSUbc9 (That would restore nuclear SUMOylation and the Ran gradient) may be used to reverse the aberrant expression of these genes. The use of NLS-Ubc9 may also be extended to the establishment of whether the DNA damage phenotypes associated with HGPS are downstream of defective nuclear SUMOylation and the closely associated Ran gradient disruption. Since the disruption of nuclear SUMOylation and the Ran gradient is closely related to the induction of ROS, it may be difficult to pinpoint which of these pathways is actually responsible for the gene expression changes. The expectation is that they are all jointly responsible. Since we have LPV, a powerful drug that mimics Progerin, at our disposal, we are in a position to follow gene expression changes as a function of time and correlate it with the prevalence of the Ran, SUMO and ROS defects. The above experiments would be critical to the understanding of how early signaling cascades unleashed by a disease mutation can impact on cellular functions, by disturbing the delicate balance of gene transcription, as well as by reducing cellular viability by compromising the integrity of the genome. After all the above represent two of the most fundamental mechanisms for progression of any genetic disease.

Thus in conclusion we propose that the cellular defects in HGPS are caused by a complex interplay of signals that involves the disruption of the Ran gradient, defective nuclear import of Ubc9, loss of nuclear SUMOylation and induction of Oxidative stress. These represent the intermediate phenomena that follow the perturbation of the nuclear lamina and lay the groundwork for developing an understanding of the cellular 
progression of HGPS. They also provide an interesting platform for investigating how some of the downstream phenotypes of HGPS namely changes in gene expression patterns, DNA damage and cellular senescence may develop. Ultimately it would be an in-depth understanding of this connection that would allow us to dissect out the fundamentals of this dreadful disease and possibly move towards a cure. 


\section{References}

Adam, S.A., Marr, R.S., and Gerace, L. (1990). Nuclear protein import in permeabilized mammalian cells requires soluble cytoplasmic factors. The Journal of Cell Biology 111, 807-816.

Alcendor, R.R., Gao, S., Zhai, P., Zablocki, D., Holle, E., Yu, X., Tian, B., Wagner, T., Vatner, S.F., and Sadoshima, J. (2007). Sirt1 regulates aging and resistance to oxidative stress in the heart. Circulation research 100, 1512-1521.

Allsopp, R.C., Vaziri, H., Patterson, C., Goldstein, S., Younglai, E.V., Futcher, A.B., Greider, C.W., and Harley, C.B. (1992). Telomere length predicts replicative capacity of human fibroblasts. Proceedings of the National Academy of Sciences 89, 10114-10118.

Barrowman, J., Hamblet, C., George, C.M., and Michaelis, S. (2008). Analysis of prelamin A biogenesis reveals the nucleus to be a CaaX processing compartment. Molecular biology of the cell 19, 5398-5408.

Bayer, P., Arndt, A., Metzger, S., Mahajan, R., Melchior, F., Jaenicke, R., and Becker, J.r. (1998). Structure determination of the small ubiquitin-related modifier SUMO-1. Journal of molecular biology $280,275-286$.

Beckman, K.B., and Ames, B.N. (1998). The free radical theory of aging matures. Physiological reviews 78, 547-581. 
Ben-Efraim, I., Frosst, P.D., and Gerace, L. (2009). Karyopherin binding interactions and nuclear import mechanism of nuclear pore complex protein Tpr. BMC cell biology 10,74 .

Benson, E.K., Lee, S.W., and Aaronson, S.A. (2010). Role of progerin-induced telomere dysfunction in HGPS premature cellular senescence. Journal of cell science 123, 26052612.

Bernad, R., van der Velde, H., Fornerod, M., and Pickersgill, H. (2004).

Nup358/RanBP2 attaches to the nuclear pore complex via association with Nup88 and Nup214/CAN and plays a supporting role in CRM1-mediated nuclear protein export. Molecular and Cellular Biology 24, 2373-2384.

Bischoff, F., Klebe, C., Kretschmer, J., Wittinghofer, A., and Ponstingl, H. (1994a). RanGAP1 induces GTPase activity of nuclear Ras-related Ran. Proceedings of the National Academy of Sciences 91, 2587-2591.

Bischoff, F., Krebber, H., Smirnova, E., Dong, W., and Ponstingl, H. (1995). Coactivation of RanGTPase and inhibition of GTP dissociation by Ran-GTP binding protein RanBP1. The EMBO Journal 14, 705.

Bischoff, F., and Ponstingl, H. (1991). Catalysis of guanine nucleotide exchange on Ran by the mitotic regulator RCC 1 . Nature $354,80-82$. 
Black, B.E., Levesque, L., Holaska, J.M., Wood, T.C., and Paschal, B.M. (1999). Identification of an NTF2-related factor that binds Ran-GTP and regulates nuclear protein export. Molecular and Cellular Biology 19, 8616.

Bodoor, K., Shaikh, S., Salina, D., Raharjo, W.H., Bastos, R., Lohka, M., and Burke, B. (1999). Sequential recruitment of NPC proteins to the nuclear periphery at the end of mitosis. Journal of cell science 112, 2253-2264.

Bossis, G., and Melchior, F. (2006). Regulation of SUMOylation by reversible oxidation of SUMO conjugating enzymes. Molecular Cell 21, 349-357.

Bridger, J.M., and Kill, I.R. (2004). Aging of Hutchinsonâ€“Gilford progeria syndrome fibroblasts is characterised by hyperproliferation and increased apoptosis. Experimental gerontology 39, 717-724.

Brown, K., Gerstberger, S., Carlson, L., Franzoso, G., and Siebenlist, U. (1995). Control of I kappa B-alpha proteolysis by site-specific, signal-induced phosphorylation. Science $267,1485-1488$.

Brunet, A., Sweeney, L.B., Sturgill, J.F., Chua, K.F., Greer, P.L., Lin, Y., Tran, H., Ross, S.E., Mostoslavsky, R., and Cohen, H.Y. (2004). Stress-dependent regulation of FOXO transcription factors by the SIRT1 deacetylase. Science Signaling 303, 2011.

Budd, S., and Nicholls, D. (1996). A reevaluation of the role of mitochondria in neuronal Ca2+ homeostasis. Journal of neurochemistry $66,403-411$. 
Cao, K., Blair, C.D., Faddah, D.A., Kieckhaefer, J.E., Olive, M., Erdos, M.R., Nabel, E.G., and Collins, F.S. (2011a). Progerin and telomere dysfunction collaborate to trigger cellular senescence in normal human fibroblasts. The Journal of clinical investigation $121,2833$.

Cao, K., Capell, B.C., Erdos, M.R., Djabali, K., and Collins, F.S. (2007). A lamin A protein isoform overexpressed in Hutchinsonâ€“Gilford progeria syndrome interferes with mitosis in progeria and normal cells. Proceedings of the National Academy of Sciences 104, 4949-4954.

Cao, K., Graziotto, J.J., Blair, C.D., Mazzulli, J.R., Erdos, M.R., Krainc, D., and Collins, F.S. (2011b). Rapamycin reverses cellular phenotypes and enhances mutant protein clearance in Hutchinson-Gilford progeria syndrome cells. Sci Transl Med 3, 89ra58.

Capel, E., Auclair, M., Caron-Debarle, M., and Capeau, J. Effects of ritonavir-boosted darunavir, atazanavir and lopinavir on adipose functions and insulin sensitivity in murine and human adipocytes. Antiviral therapy 17, 549.

Capell, B., Collins, F., and Nabel, E. (2007). Mechanisms of cardiovascular disease in accelerated aging syndromes. Circulation research 101, 13.

Capell, B.C., Erdos, M.R., Madigan, J.P., Fiordalisi, J.J., Varga, R., Conneely, K.N., Gordon, L.B., Der, C.J., Cox, A.D., and Collins, F.S. (2005). Inhibiting farnesylation of progerin prevents the characteristic nuclear blebbing of Hutchinson-Gilford progeria syndrome. Proceedings of the National Academy of Sciences of the United States of America 102, 12879-12884. 
Caron, M., Auclair, M., Donadille, B., Bereziat, V., Guerci, B., Laville, M., Narbonne, H., Bodemer, C., Lascols, O., and Capeau, J. (2007). Human lipodystrophies linked to mutations in A-type lamins and to HIV protease inhibitor therapy are both associated with prelamin A accumulation, oxidative stress and premature cellular senescence. Cell Death \& Differentiation 14, 1759-1767.

Casolari, J.M., Brown, C.R., Komili, S., West, J., Hieronymus, H., and Silver, P.A. (2004). Genome-wide localization of the nuclear transport machinery couples transcriptional status and nuclear organization. Cell 117, 427-439.

Chai, H., Yang, H., Yan, S., Li, M., Lin, P.H., Lumsden, A.B., Yao, Q., and Chen, C. (2005). Effects of 5 HIV protease inhibitors on vasomotor function and superoxide anion production in porcine coronary arteries. JAIDS Journal of Acquired Immune Deficiency Syndromes 40, 12-19.

Chakrabarti, S.R., and Nucifora, G. (1999). The leukemia-associated gene TEL encodes a transcription repressor which associates with SMRT and mSin3A. Biochemical and biophysical research communications 264, 871-877.

Chen, C.-Y., Chi, Y.-H., Mutalif, R.A., Starost, M.F., Myers, T.G., Anderson, S.A., Stewart, C.L., and Jeang, K.-T. Accumulation of the inner nuclear envelope protein sun1 is pathogenic in progeric and dystrophic laminopathies. Cell 149, 565-577.

Chen, L.B. (1988). Mitochondrial membrane potential in living cells. Annual review of cell biology 4, 155-181. 
Chinopoulos, C., Tretter, L., and Adamâ€• Vizi, V. (2002). Depolarization of In Situ Mitochondria Due to Hydrogen Peroxideâ€• Induced Oxidative Stress in Nerve Terminals. Journal of neurochemistry $73,220-228$.

Cimarosti, H., Lindberg, C., Bomholt, S.F., RÃ nn, L.C., and Henley, J.M. (2008). Increased protein SUMOylation following focal cerebral ischemia. Neuropharmacology $54,280-289$.

Coffinier, C., Hudon, S.E., Farber, E.A., Chang, S.Y., Hrycyna, C.A., Young, S.G., and Fong, L.G. (2007). HIV protease inhibitors block the zinc metalloproteinase ZMPSTE24 and lead to an accumulation of prelamin A in cells. Proceedings of the National Academy of Sciences 104, 13432-13437.

Cordes, V., Reidenbach, S., Rackwitz, H., and Franke, W. (1997). Identification of protein p270/Tpr as a constitutive component of the nuclear pore complex-attached intranuclear filaments. Journal of Cell Biology 136, 515-529.

Corrigan, D.P., Kuszczak, D., Rusinol, A.E., Thewke, D.P., Hrycyna, C.A., Michaelis, S., and Sinensky, M.S. (2005). Prelamin A endoproteolytic processing in vitro by recombinant Zmpste24. Biochemical Journal 387, 129.

Croft, J.A., Bridger, J.M., Boyle, S., Perry, P., Teague, P., and Bickmore, W.A. (1999). Differences in the localization and morphology of chromosomes in the human nucleus. The Journal of Cell Biology 145, 1119-1131. 
Csoka, A., English, S., Simkevich, C., Ginzinger, D., Butte, A., Schatten, G., Rothman, F., and Sedivy, J. (2004). Genome-scale expression profiling of Hutchinson-Gilford progeria syndrome reveals widespread transcriptional misregulation leading to mesodermal/mesenchymal defects and accelerated atherosclerosis. Aging Cell 3, 235243.

Czubryt, M.P., Austria, J.A., and Pierce, G.N. (2000). Hydrogen peroxide inhibition of nuclear protein import is mediated by the mitogen-activated protein kinase, ERK2. The Journal of Cell Biology 148, 7-16.

D'Angelo, M.A., Raices, M., Panowski, S.H., and Hetzer, M.W. (2009). Age-dependent deterioration of nuclear pore complexes causes a loss of nuclear integrity in postmitotic cells. Cell 136, 284-295.

D'Autréaux, B., and Toledano, M.B. (2007). ROS as signalling molecules: mechanisms that generate specificity in ROS homeostasis. Nature Reviews Molecular Cell Biology 8, 813-824.

Dahl, K.N., Scaffidi, P., Islam, M.F., Yodh, A.G., Wilson, K.L., and Misteli, T. (2006). Distinct structural and mechanical properties of the nuclear lamina in Hutchinsonâ€“Gilford progeria syndrome. Proceedings of the National Academy of Sciences 103, 10271-10276.

Dai, D.-F., Santana, L.F., Vermulst, M., Tomazela, D.M., Emond, M.J., MacCoss, M.J., Gollahon, K., Martin, G.M., Loeb, L.A., and Ladiges, W.C. (2009). Overexpression of 
catalase targeted to mitochondria attenuates murine cardiac aging. Circulation 119, 2789-2797.

Dai, Q., Choy, E., Chiu, V., Romano, J., Slivka, S.R., Steitz, S.A., Michaelis, S., and Philips, M.R. (1998). Mammalian prenylcysteine carboxyl methyltransferase is in the endoplasmic reticulum. Journal of Biological Chemistry 273, 15030-15034.

De Sandre-Giovannoli, A., Bernard, R., Cau, P., Navarro, C., Amiel, J., Boccaccio, I., Lyonnet, S., Stewart, C.L., Munnich, A., and Le Merrer, M. (2003). Lamin a truncation in Hutchinson-Gilford progeria. Science 300, 2055-2055.

Dechat, T., Pfleghaar, K., Sengupta, K., Shimi, T., Shumaker, D.K., Solimando, L., and Goldman, R.D. (2008). Nuclear lamins: major factors in the structural organization and function of the nucleus and chromatin. Genes \& development 22, 832-853.

Dechat, T., Shimi, T., Adam, S.A., Rusinol, A.E., Andres, D.A., Spielmann, H.P., Sinensky, M.S., and Goldman, R.D. (2007). Alterations in mitosis and cell cycle progression caused by a mutant lamin A known to accelerate human aging. Proceedings of the National Academy of Sciences 104, 4955-4960.

Dellaire, G., and Bazett-Jones, D.P. (2004). PML nuclear bodies: dynamic sensors of DNA damage and cellular stress. Bioessays 26, 963-977.

Deng, W., Baki, L., Yin, J., Zhou, H., and Baumgarten, C.M. HIV protease inhibitors elicit volume-sensitive $\mathrm{Cl}<$ sup $>\hat{a}^{\wedge}{ }^{\prime}</$ sup $>$ current in cardiac myocytes via mitochondrial ROS. Journal of molecular and cellular cardiology 49, 746-752. 
Deniaud, A. (2007). Endoplasmic reticulum stress induces calcium-dependent permeability transition, mitochondrial outer membrane permeabilization and apoptosis. Oncogene 27, 285-299.

Desterro, J.M., Rodriguez, M.S., and Hay, R.T. (1998). SUMO-1 modification of lîoBît inhibits NF-ÎoB activation. Molecular Cell 2, 233-239.

Engelsma, D., Bernad, R., Calafat, J., and Fornerod, M. (2004). Supraphysiological nuclear export signals bind CRM1 independently of RanGTP and arrest at Nup358. The EMBO Journal 23, 3643-3652.

Eriksson, M., Brown, W., Gordon, L., Glynn, M., Singer, J., Scott, L., Erdos, M., Robbins, C., Moses, T., and Berglund, P. (2003a). Recurrent de novo point mutations in lamin A cause Hutchinson-Gilford progeria syndrome.

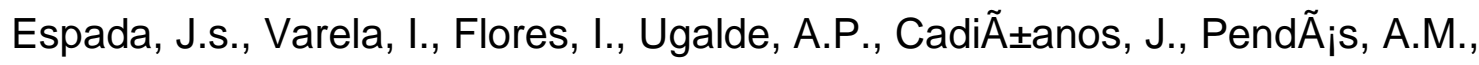
Stewart, C.L., Tryggvason, K., Blasco, M.a.A., and Freije, J.M. (2008). Nuclear envelope defects cause stem cell dysfunction in premature-aging mice. The Journal of Cell Biology 181, 27-35.

Fahrenkrog, B., and Aebi, U. (2003). The nuclear pore complex: nucleocytoplasmic transport and beyond. Nature Reviews Molecular Cell Biology 4, 757-766.

Finkel, T., and Holbrook, N.J. (2000). Oxidants, oxidative stress and the biology of ageing. NATURE-LONDON-, 239-247. 
Fong, L.G., Frost, D., Meta, M., Qiao, X., Yang, S.H., Coffinier, C., and Young, S.G. (2006). A protein farnesyltransferase inhibitor ameliorates disease in a mouse model of progeria. Science 311, 1621-1623.

Fornerod, M., Ohno, M., Yoshida, M., and Mattaj, I. (1997). CRM1 is an export receptor for leucine-rich nuclear export signals. Cell 90, 1051-1060.

Frosst, P., Guan, T., Subauste, C., Hahn, K., and Gerace, L. (2002). Tpr is localized within the nuclear basket of the pore complex and has a role in nuclear protein export. The Journal of Cell Biology 156, 617-630.

Fukuda, M., Asano, S., Nakamura, T., Adachi, M., Yoshida, M., Yanagida, M., and Nishida, E. (1997). CRM1 is responsible for intracellular transport mediated by the nuclear export signal. Nature 390, 308-311.

Goth, L.s., Shemirani, A., and KalmÃ $i r$, T. (2000). A novel catalase mutation (a GA insertion) causes the hungarian type of acatalasemia. Blood Cells, Molecules, and Diseases 26, 151-154.

Gorlich, D., and Kutay, U. (1999). Transport between the cell nucleus and the cytoplasm. Annual review of cell and developmental biology 15, 607-660.

Gorlich, D., Seewald, M.J., and Ribbeck, K. (2003). Characterization of Ran-driven cargo transport and the RanGTPase system by kinetic measurements and computer simulation. The EMBO Journal 22, 1088-1100. 
Geiss-Friedlander, R., and Melchior, F. (2007). Concepts in sumoylation: a decade on. Nature Reviews Molecular Cell Biology 8, 947-956.

Gerace, L., and Blobel, G.n. (1980). The nuclear envelope lamina is reversibly depolymerized during mitosis. Cell 19, 277.

Girdwood, D., Bumpass, D., Vaughan, O.A., Thain, A., Anderson, L.A., Snowden, A.W., Garcia-Wilson, E., Perkins, N.D., and Hay, R.T. (2003). P300 transcriptional repression is mediated by SUMO modification. Molecular Cell 11, 1043-1054.

Glynn, M.W., and Glover, T.W. (2005). Incomplete processing of mutant lamin A in Hutchinsonâ€“Gilford progeria leads to nuclear abnormalities, which are reversed by farnesyltransferase inhibition. Human molecular genetics 14, 2959-2969.

Goldman, R., Gruenbaum, Y., Moir, R., Shumaker, D., and Spann, T. (2002). Nuclear lamins: building blocks of nuclear architecture. Genes \& development 16, 533-547.

Goldman, R., Shumaker, D., Erdos, M., Eriksson, M., Goldman, A., Gordon, L., Gruenbaum, Y., Khuon, S., Mendez, M., and Varga, R. (2004). Accumulation of mutant lamin A causes progressive changes in nuclear architecture in Hutchinson-Gilford progeria syndrome. Proceedings of the National Academy of Sciences 101, 8963-8968.

Gordon, L.B., Kleinman, M.E., Miller, D.T., Neuberg, D.S., Giobbie-Hurder, A., GerhardHerman, M., Smoot, L.B., Gordon, C.M., Cleveland, R., and Snyder, B.D. Clinical trial of a farnesyltransferase inhibitor in children with Hutchinsonâ€"Gilford progeria syndrome. Proceedings of the National Academy of Sciences. 
Görlich, D., Seewald, M., and Ribbeck, K. (2003). Characterization of Ran-driven cargo transport and the RanGTPase system by kinetic measurements and computer simulation. The EMBO Journal 22, 1088-1100.

Grunwald, M., and Bono, F. Structure of Importin13â€“Ubc9 complex: nuclear import and release of a key regulator of sumoylation. The EMBO Journal 30, 427-438.

Gruenbaum, Y., Goldman, R.D., Meyuhas, R., Mills, E., Margalit, A., Fridkin, A., Dayani, Y., Prokocimer, M., and Enosh, A. (2003). The nuclear lamina and its functions in the nucleus. International review of cytology 226, 1-62.

Gruenbaum, Y., Margalit, A., Goldman, R.D., Shumaker, D.K., and Wilson, K.L. (2005). The nuclear lamina comes of age. Nature Reviews Molecular Cell Biology 6, 21-31.

Guo, D., Li, M., Zhang, Y., Yang, P., Eckenrode, S., Hopkins, D., Zheng, W., Purohit, S., Podolsky, R.H., and Muir, A. (2004). A functional variant of SUMO4, a new lîBît modifier, is associated with type 1 diabetes. Nature genetics $36,837-841$.

Hagen, T.M., Yowe, D.L., Bartholomew, J.C., Wehr, C.M., Do, K.L., Park, J.-Y., and Ames, B.N. (1997). Mitochondrial decay in hepatocytes from old rats: membrane potential declines, heterogeneity and oxidants increase. Proceedings of the National Academy of Sciences 94, 3064-3069.

Han, X., Feng, X., Rattner, J., Smith, H., Bose, P., Suzuki, K., Soliman, M., Scott, M., Burke, B., and Riabowol, K. (2008). Tethering by lamin A stabilizes and targets the ING1 tumour suppressor. Nature Cell Biology. 
Hang, J., and Dasso, M. (2002). Association of the human SUMO-1 protease SENP2 with the nuclear pore. Journal of Biological Chemistry 277, 19961-19966.

Haque, F., Mazzeo, D., Patel, J.T., Smallwood, D.T., Ellis, J.A., Shanahan, C.M., and Shackleton, S. Mammalian SUN protein interaction networks at the inner nuclear membrane and their role in laminopathy disease processes. Journal of Biological Chemistry 285, 3487-3498.

Hardeland, U., Bentele, M., Lettieri, T., Steinacher, R., Jiricny, J., and Schär, P. (2001). Thymine DNA glycosylase. Progress in nucleic acid research and molecular biology 68 , 235-253.

Hardeland, U., Steinacher, R., Jiricny, J., and SchÃar, P. (2002). Modification of the human thymine-DNA glycosylase by ubiquitin-like proteins facilitates enzymatic turnover. The EMBO Journal 21, 1456-1464.

Harding, H.P., Zhang, Y., Zeng, H., Novoa, I., Lu, P.D., Calfon, M., Sadri, N., Yun, C., Popko, B., and Paules, R. (2003). An integrated stress response regulates amino acid metabolism and resistance to oxidative stress. Molecular cell 11, 619-633.

Hatefi, Y. (1985). The mitochondrial electron transport and oxidative phosphorylation system. Annual review of biochemistry 54, 1015-1069.

Haynes, C.M., Titus, E.A., and Cooper, A.A. (2004). Degradation of misfolded proteins prevents ER-derived oxidative stress and cell death. Molecular cell 15, 767-776. 
HENNEKAM, R. (2006). Hutchinson-Gilford progeria syndrome: review of the phenotype. American journal of medical genetics. Part A 140, 2603-2624.

Hernandez, L., Roux, K.J., Wong, E.S.M., Mounkes, L.C., Mutalif, R., Navasankari, R., Rai, B., Cool, S., Jeong, J.-W., and Wang, H. Functional coupling between the extracellular matrix and nuclear lamina by Wnt signaling in progeria. Developmental Cell $19,413-425$.

Hetzer, M., Gruss, O.J., and Mattaj, I.W. (2002). The Ran GTPase as a marker of chromosome position in spindle formation and nuclear envelope assembly. Nature Cell Biology 4, E177-E184.

Holtz, D., Tanaka, R.A., Hartwig, J., and McKeon, F. (1989). The CaaX motif of lamin A functions in conjunction with the nuclear localization signal to target assembly to the nuclear envelope. Cell 59, 969-977.

Huang, S., Chen, L., Libina, N., Janes, J., Martin, G.M., Campisi, J., and Oshima, J. (2005). Correction of cellular phenotypes of Hutchinson-Gilford Progeria cells by RNA interference. Human genetics 118, 444-450.

Huang, S., Risques, R.A., Martin, G.M., Rabinovitch, P.S., and Oshima, J. (2008). Accelerated telomere shortening and replicative senescence in human fibroblasts overexpressing mutant and wild-type lamin A. Experimental cell research 314, 82-91.

Hunt, C.R., Sim, J.E., Sullivan, S.J., Featherstone, T., Golden, W., Von Kapp-Herr, C., Hock, R.A., Gomez, R.A., Parsian, A.J., and Spitz, D.R. (1998). Genomic instability and 
catalase gene amplification induced by chronic exposure to oxidative stress. Cancer research 58, 3986-3992.

Hutten, S., Flotho, A., Melchior, F., and Kehlenbach, R. (2008). The Nup358-RanGAP Complex Is Required for Efficient Importin \{alpha\}/\{beta\}-dependent Nuclear Import. Molecular biology of the cell 19, 2300.

Ibrahim, M.X., Sayin, V.I., Akula, M.K., Liu, M., Fong, L.G., Young, S.G., and Bergo, M.O. (2013). Targeting Isoprenylcysteine Methylation Ameliorates Disease in a Mouse Model of Progeria. Science (New York, NY).

Imai, S.-i., Nishibayashi, S., Takao, K., Tomifuji, M., Fujino, T., Hasegawa, M., and Takano, T. (1997). Dissociation of Oct-1 from the nuclear peripheral structure induces the cellular aging-associated collagenase gene expression. Molecular biology of the cell 8, 2407-2419.

Jiang, B., Hebert, V.Y., Li, Y., Mathis, J.M., Alexander, J.S., and Dugas, T.R. (2007). HIV antiretroviral drug combination induces endothelial mitochondrial dysfunction and reactive oxygen species production, but not apoptosis. Toxicology and applied pharmacology 224, 60-71.

Johnson, E.S., and Gupta, A.A. (2001). An E3-like factor that promotes SUMO conjugation to the yeast septins. Cell 106, 735-744. 
Jornot, L., Maechler, P., Wollheim, C.B., and Junod, A.F. (1999). Reactive oxygen metabolites increase mitochondrial calcium in endothelial cells: implication of the $\mathrm{Ca} 2+/ \mathrm{Na}+$ exchanger. Journal of cell science 112, 1013-1022.

Kagey, M.H., Melhuish, T.A., and Wotton, D. (2003). The polycomb protein Pc2 is a SUMO E3. Cell 113, 127-137.

Kelley, J., and Paschal, B. (2007). Hyperosmotic stress signaling to the nucleus disrupts the Ran gradient and the production of RanGTP. Molecular biology of the cell 18, 4365 .

Kelley, J.B., Datta, S., Snow, C.J., Chatterjee, M., Ni, L., Spencer, A., Yang, C.S., Cubenas-Potts, C., Matunis, M.J., and Paschal, B.M. The Defective Nuclear Lamina in Hutchinson-Gilford Progeria Syndrome Disrupts the Nucleocytoplasmic Ran Gradient and Inhibits Nuclear Localization of Ubc9. Molecular and Cellular Biology 31, 33783395.

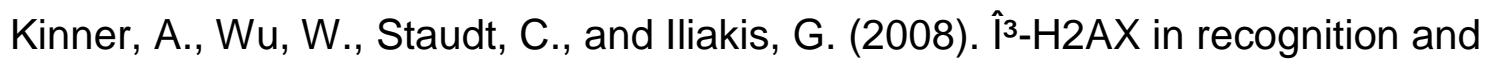
signaling of DNA double-strand breaks in the context of chromatin. Nucleic Acids Research 36, 5678-5694.

Knipscheer, P., Flotho, A., Klug, H., Olsen, J., van Dijk, W., Fish, A., Johnson, E., Mann, M., Sixma, T., and Pichler, A. (2008). Ubc9 Sumoylation Regulates SUMO Target Discrimination. Molecular Cell 31, 371-382. 
Kodiha, M., Chu, A., Matusiewicz, N., and Stochaj, U. (2004). Multiple mechanisms promote the inhibition of classical nuclear import upon exposure to severe oxidative stress. Cell Death \& Differentiation 11, 862-874.

Koepke, J.I., Nakrieko, K.A., Wood, C.S., Boucher, K.K., Terlecky, L.J., Walton, P.A., and Terlecky, S.R. (2007). Restoration of peroxisomal catalase import in a model of human cellular aging. Traffic 8, 1590-1600.

Koepke, J.I., Wood, C.S., Terlecky, L.J., Walton, P.A., and Terlecky, S.R. (2008). Progeric effects of catalase inactivation in human cells. Toxicology and applied pharmacology 232, 99-108.

Kops, G.J., Dansen, T.B., Polderman, P.E., Saarloos, I., Wirtz, K.W., Coffer, P.J., Huang, T.-T., Bos, J.L., Medema, R.H., and Burgering, B.M. (2002). Forkhead transcription factor FOXO3a protects quiescent cells from oxidative stress. Nature 419, 316-321.

Kubben, N., Adriaens, M., Meuleman, W., Voncken, J.W., van Steensel, B., and Misteli, T. (2012). Mapping of lamin A-and progerin-interacting genome regions. Chromosoma $121,447-464$.

Lagathu, C., Eustace, B., Prot, M., Frantz, D., Gu, Y., Bastard, J.P., Maachi, M., Azoulay, S., Briggs, M., and Caron, M. (2007). Some HIV antiretrovirals increase oxidative stress and alter chemokine, cytokine or adiponectin production in human adipocytes and macrophages. Antiviral therapy 12, 489. 
Lambeth, J.D. (2004). NOX enzymes and the biology of reactive oxygen. Nature Reviews Immunology 4, 181-189.

Laszlo, G., Peter, R., and Aniko, P. (2004). Catalase enzyme mutations and their association with diseases. Molecular Diagnosis 8, 141-149.

Lee, G., Melchior, F., Matunis, M., Mahajan, R., Tian, Q., and Anderson, P. (1998). Modification of Ran GTPase-activating protein by the small ubiquitin-related modifier SUMO-1 requires Ubc9, an E2-type ubiquitin-conjugating enzyme homologue. Journal of Biological Chemistry 273, 6503-6507.

Lefavre, C., Auclair, M., Boccara, F., Bastard, J.P., Capeau, J., Vigouroux, C., and Caron-Debarle, M. Premature Senescence of Vascular Cells Is Induced by HIV Protease Inhibitors Implication of Prelamin A and Reversion by Statin. Arteriosclerosis, thrombosis, and vascular biology 30, 2611-2620.

Legakis, J.E., Koepke, J.I., Jedeszko, C., Barlaskar, F., Terlecky, L.J., Edwards, H.J., Walton, P.A., and Terlecky, S.R. (2002). Peroxisome senescence in human fibroblasts. Molecular biology of the cell 13, 4243-4255.

Lemasters, J.J., Nieminen, A.-L., Qian, T., Trost, L.C., Elmore, S.P., Nishimura, Y., Crowe, R.A., Cascio, W.E., Bradham, C.A., and Brenner, D.A. (1998). The mitochondrial permeability transition in cell death: a common mechanism in necrosis, apoptosis and autophagy. Biochimica et biophysica acta 1366, 177. 
Leyssens, A., Nowicky, A.V., Patterson, L., Crompton, M., and Duchen, M. (1996). The relationship between mitochondrial state, ATP hydrolysis,[Mg2+] i and [Ca2+] i studied in isolated rat cardiomyocytes. The Journal of physiology 496, 111-128.

Li, H.Y., Wirtz, D., and Zheng, Y. (2003). A mechanism of coupling RCC1 mobility to RanGTP production on the chromatin in vivo. The Journal of Cell Biology 160, 635-644.

Lin, D.-Y., Huang, Y.-S., Jeng, J.-C., Kuo, H.-Y., Chang, C.-C., Chao, T.-T., Ho, C.-C., Chen, Y.-C., Lin, T.-P., and Fang, H.-I. (2006). Role of SUMO-interacting motif in Daxx SUMO modification, subnuclear localization, and repression of sumoylated transcription factors. Molecular Cell 24, 341-354.

Liu, B., Wang, J., Chan, K., Tjia, W., Deng, W., Guan, X., Huang, J., Li, K., Chau, P., and Chen, D. (2005). Genomic instability in laminopathy-based premature aging. Nature medicine 11, 780-785.

Liu, G.-H., Barkho, B.Z., Ruiz, S., Diep, D., Qu, J., Yang, S.-L., Panopoulos, A.D., Suzuki, K., Kurian, L., and Walsh, C. (2011). Recapitulation of premature ageing with iPSCs from Hutchinson-Gilford progeria syndrome. Nature 472, 221-225.

Liu, Y., Rusinol, A., Sinensky, M., Wang, Y., and Zou, Y. (2006). DNA damage responses in progeroid syndromes arise from defective maturation of prelamin $\mathrm{A}$. Journal of cell science 119,4644 . 
Liu, Y., Wang, Y., Rusinol, A.E., Sinensky, M.S., Liu, J., Shell, S.M., and Zou, Y. (2008). Involvement of xeroderma pigmentosum group A (XPA) in progeria arising from defective maturation of prelamin A. The FASEB Journal 22, 603-611.

Maechler, P., Jornot, L., and Wollheim, C.B. (1999). Hydrogen peroxide alters mitochondrial activation and insulin secretion in pancreatic beta cells. Journal of Biological Chemistry 274, 27905-27913.

Mahajan, R., Delphin, C., Guan, T., Gerace, L., and Melchior, F. (1997). A small ubiquitin-related polypeptide involved in targeting RanGAP1 to nuclear pore complex protein RanBP2. Cell 88, 97-108.

Mailloux, R.J., and Harper, M.-E. Mitochondrial proticity and ROS signaling: lessons from the uncoupling proteins. Trends in Endocrinology \& Metabolism.

Malhas, A.N., Lee, C.F., and Vaux, D.J. (2009). Lamin B1 controls oxidative stress responses via Oct-1. The Journal of cell biology 184, 45-55.

Malhotra, J.D., and Kaufman, R.J. (2007). Endoplasmic reticulum stress and oxidative stress: a vicious cycle or a double-edged sword? Antioxidants \& redox signaling 9, 2277-2294.

Mallampalli, M.P., Huyer, G., Bendale, P., Gelb, M.H., and Michaelis, S. (2005). Inhibiting farnesylation reverses the nuclear morphology defect in a HeLa cell model for Hutchinson-Gilford progeria syndrome. Proceedings of the National Academy of Sciences of the United States of America 102, 14416-14421. 
Manza, L.L., Codreanu, S.G., Stamer, S.L., Smith, D.L., Wells, K.S., Roberts, R.L., and Liebler, D.C. (2004). Global shifts in protein sumoylation in response to electrophile and oxidative stress. Chemical research in toxicology $17,1706-1715$.

Mariño, G., Ugalde, A.P., Fernández, Á.F., Osorio, F.G., Fueyo, A., Freije, J.M., and López-Otín, C. (2010). Insulin-like growth factor 1 treatment extends longevity in a mouse model of human premature aging by restoring somatotroph axis function. Proceedings of the National Academy of Sciences 107, 16268-16273.

Mates, J.M., Pérez-Gómez, C., and De Castro, I.N. (1999). Antioxidant enzymes and human diseases. Clinical biochemistry 32, 595-603.

Matsuura, Y., and Stewart, M. (2005). Nup50/Npap60 function in nuclear protein import complex disassembly and importin recycling. The EMBO Journal 24, 3681-3689.

Matunis, M.J., Coutavas, E., and Blobel, G. (1996). A novel ubiquitin-like modification modulates the partitioning of the Ran-GTPase-activating protein RanGAP1 between the cytosol and the nuclear pore complex. The Journal of Cell Biology 135, 1457-1470.

McClintock, D., Gordon, L.B., and Djabali, K. (2006). Hutchinson-Gilford progeria mutant lamin A primarily targets human vascular cells as detected by an anti-Lamin A G608G antibody. Proceedings of the National Academy of Sciences of the United States of America 103, 2154-2159.

Melchior, F. (1995). GTP hydrolysis by Ran occurs at the nuclear pore complex in an early step of protein import. Journal of Cell Biology $131,571-581$. 
Meshorer, E., and Gruenbaum, Y. (2008). Gone with the Wnt/Notch: stem cells in laminopathies, progeria, and aging. The Journal of Cell Biology 181, 9-13.

Mingot, J., Kostka, S., Kraft, R., Hartmann, E., and Görlich, D. (2001). Importin 13: a novel mediator of nuclear import and export. The EMBO Journal 20, 3685-3694.

Miyamoto, Y., Saiwaki, T., Yamashita, J., Yasuda, Y., Kotera, I., Shibata, S., Shigeta, M., Hiraoka, Y., Haraguchi, T., and Yoneda, Y. (2004). Cellular stresses induce the nuclear accumulation of importin \{alpha\} and cause a conventional nuclear import block. Science Signalling 165, 617.

Mohr, D., Frey, S., Fischer, T., Güttler, T., and Görlich, D. (2009). Characterisation of the passive permeability barrier of nuclear pore complexes. The EMBO journal 28 , 2541-2553.

Moir, R.D., Yoon, M., Khuon, S., and Goldman, R.D. (2000). Nuclear Lamins a and B1 Different Pathways of Assembly during Nuclear Envelope Formation in Living Cells. The Journal of Cell Biology 151, 1155-1168.

Mounkes, L., Kozlov, S., Burke, B., and Stewart, C.L. (2003). The laminopathies: nuclear structure meets disease. Current opinion in genetics \& development 13, 223230.

Mukhopadhyay, D., and Dasso, M. (2007). Modification in reverse: the SUMO proteases. Trends in Biochemical Sciences 32, 286-295. 
Nagai, M., and Yoneda, Y. (2012). Downregulation of the small GTPase Ras-related nuclear protein accelerates cellular aging. Biochimica et Biophysica Acta (BBA)-General Subjects.

Nemergut, M., Mizzen, C., Stukenberg, T., Allis, C., and Macara, I. (2001). Chromatin docking and exchange activity enhancement of RCC1 by histones $\mathrm{H} 2 \mathrm{~A}$ and $\mathrm{H} 2 \mathrm{~B}$. pp. 1540-1543.

Nemoto, S., and Finkel, T. (2002). Redox regulation of forkhead proteins through a p66shc-dependent signaling pathway. Science Signaling 295, 2450.

Ohtsubo, M., Okazaki, H., and Nishimoto, T. (1989). The RCC1 protein, a regulator for the onset of chromosome condensation locates in the nucleus and binds to DNA. The Journal of Cell Biology 109, 1389-1397.

Orrell, R., King, A., Hilton, D., Campbell, M., Lane, R., and De Belleroche, J. (1995). Familial amyotrophic lateral sclerosis with a point mutation of SOD-1: intrafamilial heterogeneity of disease duration associated with neurofibrillary tangles. Journal of Neurology, Neurosurgery \& Psychiatry 59, 266-270.

Osorio, F.G., Navarro, C.L., Cadiñanos, J., López-Mejía, I.C., Quirós, P.M., Bartoli, C., Rivera, J., Tazi, J., Guzmán, G., and Varela, I. (2011). Splicing-directed therapy in a new mouse model of human accelerated aging. Science translational medicine 3, 106ra107-106ra107. 
Panté, N., Bastos, R., McMorrow, I., Burke, B., and Aebi, U. (1994). Interactions and three-dimensional localization of a group of nuclear pore complex proteins. The Journal of cell biology $126,603-617$.

Paschal, B. (1995). Identification of NTF2, a cytosolic factor for nuclear import that interacts with nuclear pore complex protein p62. Journal of Cell Biology 129, 925-937.

Paschal, B.M., and Gerace, L. (1995). Identification of NTF2, a cytosolic factor for nuclear import that interacts with nuclear pore complex protein p62. The Journal of cell biology 129, 925-937.

Pekovic, V., Gibbsâ€• Seymour, I., Markiewicz, E., Alzoghaibi, F., Benham, A.M., Edwards, R., Wenhert, M., von Zglinicki, T., and Hutchison, C.J. Conserved cysteine residues in the mammalian lamin A tail are essential for cellular responses to ROS generation. Aging Cell 10, 1067-1079.

Pemberton, L., and Paschal, B. (2005). Mechanisms of receptor-mediated nuclear import and nuclear export. Traffic 6, 187-198.

Petrini, J.H., and Stracker, T.H. (2003). The cellular response to DNA double-strand breaks: defining the sensors and mediators. Trends in Cell Biology 13, 458-462.

Pichler, A., Gast, A., Seeler, J., Dejean, A., and Melchior, F. (2002). The nucleoporin RanBP2 has SUMO1 E3 ligase activity. Cell 108, 109-120.

Pichler, A., and Melchior, F. (2002). Ubiquitin Related Modifier SUMO1 and Nucleocytoplasmic Transport. Traffic 3, 381-387. 
Prokocimer, M., Barka, R., and Gruenbaum, Y. (2013). Hutchinson-Gilford progeria syndrome through the lens of transcription. Aging Cell.

Rangasamy, D., Woytek, K., Khan, S.A., and Wilson, V.G. (2000). SUMO-1 modification of bovine papillomavirus E1 protein is required for intranuclear accumulation. Journal of Biological Chemistry 275, 37999-38004.

Reddy, K., Zullo, J., Bertolino, E., and Singh, H. (2008). Transcriptional repression mediated by repositioning of genes to the nuclear lamina. Nature 452, 243-247.

Ren, M., Drivas, G., D'Eustachio, P., and Rush, M.G. (1993). Ran/TC4: a small nuclear GTP-binding protein that regulates DNA synthesis. The Journal of cell biology 120 , 313323.

Reverter, D., and Lima, C. (2005). Insights into E3 ligase activity revealed by a SUMORanGAP1-Ubc9-Nup358 complex. Nature 435, 687-692.

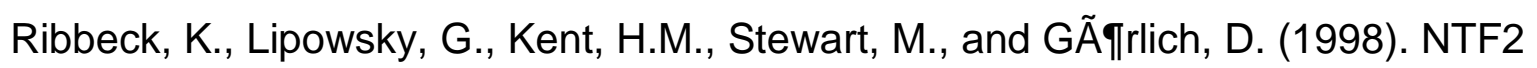
mediates nuclear import of Ran. The EMBO Journal 17, 6587-6598.

Richards, S.A., Muter, J., Ritchie, P., Lattanzi, G., and Hutchison, C.J. The accumulation of un-repairable DNA damage in laminopathy progeria fibroblasts is caused by ROS generation and is prevented by treatment with $\mathrm{N}$-acetyl cysteine. Human molecular genetics 20, 3997-4004.

Ris, H. (1989). Three-dimensional imaging of cell ultrastructure with high resolution low voltage SEM. In Inst. Phys. Conf. Ser, pp. 657-662. 
Rodriguez, S., Coppedè, F., Sagelius, H., and Eriksson, M. (2009). Increased expression of the Hutchinson-Gilford progeria syndrome truncated lamin A transcript during cell aging. European journal of human genetics 17, 928-937.

Rogakou, E.P., Boon, C., Redon, C., and Bonner, W.M. (1999). Megabase chromatin domains involved in DNA double-strand breaks in vivo. The Journal of Cell Biology 146, 905-916.

Saitoh, H., and Hinchey, J. (2000). Functional heterogeneity of small ubiquitin-related protein modifiers SUMO-1 versus SUMO-2/3. Journal of Biological Chemistry 275, $6252-6258$.

Saitoh, H., Pizzi, M., and Wang, J. (2002). Perturbation of SUMOlation enzyme Ubc9 by distinct domain within nucleoporin RanBP2/Nup358. Journal of Biological Chemistry $277,4755-4763$.

Saitoh, H., Pu, R., Cavenagh, M., and Dasso, M. (1997). RanBP2 associates with Ubc9p and a modified form of RanGAP1. (National Acad Sciences), pp. 3736-3741.

Scaffidi, P., and Misteli, T. (2005). Reversal of the cellular phenotype in the premature aging disease Hutchinson-Gilford progeria syndrome. Nature medicine 11, 440-445.

Scaffidi, P., and Misteli, T. (2006). Lamin A-dependent nuclear defects in human aging. (American Association for the Advancement of Science), pp. 1059-1063.

Scaffidi, P., and Misteli, T. (2008). Lamin A-dependent misregulation of adult stem cells associated with accelerated ageing. Nature Cell Biology 10, 452-459. 
Schirmer, E., and Gerace, L. (2005). The nuclear membrane proteome: extending the envelope. Trends in Biochemical Sciences 30, 551-558.

Schmidt, W.K., Tam, A., Fujimura-Kamada, K., and Michaelis, S. (1998). Endoplasmic reticulum membrane localization of Rce1p and Ste24p, yeast proteases involved in carboxyl-terminal CAAX protein processing and amino-terminal a-factor cleavage. Proceedings of the National Academy of Sciences 95, 11175-11180.

Schrader, N., Koerner, C., Koessmeier, K., Bangert, J., Wittinghofer, A., Stoll, R., and Vetter, I. (2008). The Crystal Structure of the Ran-Nup153ZnF2 Complex: a General Ran Docking Site at the Nuclear Pore Complex. Structure 16, 1116-1125.

Schriner, S.E., Linford, N.J., Martin, G.M., Treuting, P., Ogburn, C.E., Emond, M., Coskun, P.E., Ladiges, W., Wolf, N., and Van Remmen, H. (2005). Extension of murine life span by overexpression of catalase targeted to mitochondria. Science 308,1909 1911.

Schultz, L.B., Chehab, N.H., Malikzay, A., and Halazonetis, T.D. (2000). p53 binding protein 1 (53BP1) is an early participant in the cellular response to DNA double-strand breaks. The Journal of Cell Biology 151, 1381-1390.

Schwoebel, E.D., Ho, T.H., and Moore, M.S. (2002). The mechanism of inhibition of Ran-dependent nuclear transport by cellular ATP depletion. The Journal of Cell Biology $157,963-974$. 
Scott, I., and Nicholls, D. (1980). Energy transduction in intact synaptosomes. Influence of plasma-membrane depolarization on the respiration and membrane potential of internal mitochondria determined in situ. Biochemical Journal 186, 21.

Sedelnikova, O.A., Redon, C.E., Dickey, J.S., Nakamura, A.J., Georgakilas, A.G., and Bonner, W.M. (2010). Role of oxidatively induced DNA lesions in human pathogenesis. Mutation Research/Reviews in Mutation Research 704, 152-159.

Shang, J. (2005). Quantitative measurement of events in the mammalian unfolded protein response. Methods 35, 390-394.

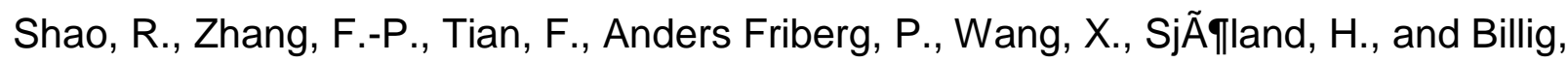
H.k. (2004). Increase of SUMO-1 expression in response to hypoxia: direct interaction with HIF-1 Î in adult mouse brain and heart in vivo. FEBS letters 569, 293-300.

Shen, L., Geoffroy, M., Jaffray, E., and Hay, R. (2009). Characterization of SENP7, a SUMO-2/3-specific isopeptidase. Biochem. J 421, 223-230.

Shen, T.H., Lin, H.-K., Scaglioni, P.P., Yung, T.M., and Pandolfi, P.P. (2006). The mechanisms of PML-nuclear body formation. Molecular Cell 24, 331-339.

Shiio, Y., and Eisenman, R.N. (2003). Histone sumoylation is associated with transcriptional repression. Proceedings of the National Academy of Sciences 100, $13225-13230$.

Shumaker, D., Dechat, T., Kohlmaier, A., Adam, S., Bozovsky, M., Erdos, M., Eriksson, M., Goldman, A., Khuon, S., and Collins, F. (2006). Mutant nuclear lamin A leads to 
progressive alterations of epigenetic control in premature aging. Proceedings of the National Academy of Sciences 103, 8703-8708.

Smith, A., Brownawell, A., and Macara, I.G. (1998). Nuclear import of Ran is mediated by the transport factor NTF2. Current biology 8, 1403-1406, S1401.

Snow, C.J., Dar, A., Dutta, A., Kehlenbach, R.H., and Paschal, B.M. (2013). Defective nuclear import of Tpr in Progeria reflects the Ran sensitivity of large cargo transport. The Journal of cell biology 201, 541-557.

Song, J., Zhang, Z., Hu, W., and Chen, Y. (2005). Small Ubiquitin-like Modifier (SUMO) Recognition of a SUMO Binding Motif. Journal of Biological Chemistry 280, 4012240129.

Spitz, D., Li, G., McCormick, M., Sun, Y., and Oberley, L. (1988a). The isolation and partial characterization of stable $\mathrm{H} 2 \mathrm{O} 2$-resistant variants of Chinese hamster fibroblasts. Basic life sciences 49, 549 .

Spitz, D.R., Li, G.C., McCormick, M.L., Sun, Y., and Oberley, L.W. (1988b). Stable $\mathrm{H} 2 \mathrm{O} 2$-resistant variants of Chinese hamster fibroblasts demonstrate increases in catalase activity. Radiat Res 114, 114-124.

Stewart, M. (2007). Molecular mechanism of the nuclear protein import cycle. Nature Reviews Molecular Cell Biology 8, 195-208. 
Stochaj, U., Rassadi, R., and Chiu, J. (2000). Stress-mediated inhibition of the classical nuclear protein import pathway and nuclear accumulation of the small GTPase Gsp1p. The FASEB Journal 14, 2130-2132.

Sullivan, T., Escalante-Alcalde, D., Bhatt, H., Anver, M., Bhat, N., Nagashima, K., Stewart, C.L., and Burke, B. (1999). Loss of A-type lamin expression compromises nuclear envelope integrity leading to muscular dystrophy. The Journal of Cell Biology 147, 913-920.

Szabo, I., and Zoratti, M. (1991). The giant channel of the inner mitochondrial membrane is inhibited by cyclosporin A. Journal of Biological Chemistry 266, 33763379.

Szabo, I., and Zoratti, M. (1992). The mitochondrial megachannel is the permeability transition pore. Journal of bioenergetics and biomembranes 24, 111-117.

Tachibana, T., Imamoto, N., Seino, H., Nishimoto, T., and Yoneda, Y. (1994). Loss of RCC1 leads to suppression of nuclear protein import in living cells. Journal of Biological Chemistry 269, 24542-24545.

Tan, S., Sagara, Y., Liu, Y., Maher, P., and Schubert, D. (1998). The regulation of reactive oxygen species production during programmed cell death. The Journal of cell biology $141,1423-1432$.

Toth, J.I., Yang, S.H., Qiao, X., Beigneux, A.P., Gelb, M.H., Moulson, C.L., Miner, J.H., Young, S.G., and Fong, L.G. (2005). Blocking protein farnesyltransferase improves 
nuclear shape in fibroblasts from humans with progeroid syndromes. Proceedings of the National Academy of Sciences of the United States of America 102, 12873-12878.

Tran, E.J., and Wente, S.R. (2006). Dynamic nuclear pore complexes: life on the edge. Cell $125,1041-1053$.

Treuting, P.M., Linford, N.J., Knoblaugh, S.E., Emond, M., Morton, J.F., Martin, G.M., Rabinovitch, P.S., and Ladiges, W.C. (2008). Reduction of age-associated pathology in old mice by overexpression of catalase in mitochondria. The Journals of Gerontology Series A: Biological Sciences and Medical Sciences 63, 813-822.

Uchimura, Y., Ichimura, T., Uwada, J., Tachibana, T., Sugahara, S., Nakao, M., and Saitoh, H. (2006). Involvement of SUMO modification in MBD1-and MCAF1-mediated heterochromatin formation. Journal of Biological Chemistry 281, 23180-23190.

Van der Heide, L.P., Hoekman, M.F., and Smidt, M.P. (2004). The ins and outs of FoxO shuttling: mechanisms of FoxO translocation and transcriptional regulation. Biochemical Journal 380, 297.

Varela, I., Pereira, S., Ugalde, A.P., Navarro, C.L., Suárez, M.F., Cau, P., Cadiñanos, J., Osorio, F.G., Foray, N., and Cobo, J. (2008). Combined treatment with statins and aminobisphosphonates extends longevity in a mouse model of human premature aging. Nature medicine 14, 767-772.

Verniquet, F., Gaillard, J., Neuburger, M., and Douce, R. (1991). Rapid inactivation of plant aconitase by hydrogen peroxide. Biochemical Journal 276, 643. 
Vincent, S., Tourniaire, F., El Yazidi, C.M., Compe, E., Manches, O., Plannels, R., and Roche, R. (2004). Nelfinavir induces necrosis of 3T3F44-2A adipocytes by oxidative stress. JAIDS Journal of Acquired Immune Deficiency Syndromes 37, 1556-1562.

Viteri, G., Chung, Y.W., and Stadtman, E.R. Effect of progerin on the accumulation of oxidized proteins in fibroblasts from Hutchinson Gilford progeria patients. Mechanisms of ageing and development 131, 2-8.

Walther, T., Pickersgill, H., Cordes, V., Goldberg, M., Allen, T., Mattaj, I., and Fornerod, M. (2002). The cytoplasmic filaments of the nuclear pore complex are dispensable for selective nuclear protein import. The Journal of Cell Biology 158, 63.

Wang, X., Mu, H., Chai, H., Liao, D., Yao, Q., and Chen, C. (2007). Human immunodeficiency virus protease inhibitor ritonavir inhibits cholesterol efflux from human macrophage-derived foam cells. The American journal of pathology 171, 304-314.

Weber, K., Plessmann, U., and Traub, P. (1989). Maturation of nuclear lamin A involves a specific carboxy-terminal trimming, which removes the polyisoprenylation site from the precursor; implications for the structure of the nuclear lamina. FEBS letters 257,411 414.

Wenzel, V., Roedl, D., Gabriel, D., Gordon, L.B., Herlyn, M., Schneider, R., Ring, J., and Djabali, K. (2012). Naïve adult stem cells from patients with Hutchinson-Gilford progeria syndrome express low levels of progerin in vivo. Biology open 1, 516-526. 
Werner, A., Flotho, A., and Melchior, F. The RanBP2/RanGAP1â^—SUMO1/Ubc9 Complex Is a Multisubunit SUMO E3 Ligase. Molecular Cell.

Wong, C.-H., Chan, H., Ho, C.-Y., Lai, S.-K., Chan, K.-S., Koh, C.-G., and Li, H.-Y. (2008). Apoptotic histone modification inhibits nuclear transport by regulating RCC1. Nature cell biology 11, 36-45.

Wu, J., Matunis, M., Kraemer, D., Blobel, G., and Coutavas, E. (1995). Nup358, a cytoplasmically exposed nucleoporin with peptide repeats, Ran-GTP binding sites, zinc fingers, a cyclophilin A homologous domain, and a leucine-rich region. Journal of Biological Chemistry 270, 14209.

Xiong, Z.-M., LaDana, C., Wu, D., and Cao, K. (2013). An inhibitory role of progerin in the gene induction network of adipocyte differentiation from iPS cells. Aging (Albany NY) 5, 288.

Yang, S.H., Meta, M., Qiao, X., Frost, D., Bauch, J., Coffinier, C., Majumdar, S., Bergo, M.O., Young, S.G., and Fong, L.G. (2006). A farnesyltransferase inhibitor improves disease phenotypes in mice with a Hutchinson-Gilford progeria syndrome mutation. Journal of Clinical Investigation 116, 2115.

Yang, W., Sheng, H., Warner, D.S., and Paschen, W. (2007a). Transient global cerebral ischemia induces a massive increase in protein sumoylation. Journal of Cerebral Blood Flow \& Metabolism 28, 269-279. 
Yang, W., Sheng, H., Warner, D.S., and Paschen, W. (2008). Transient focal cerebral ischemia induces a dramatic activation of small ubiquitin-like modifier conjugation. Journal of Cerebral Blood Flow \& Metabolism 28, 892-896.

Yang, Y., Fu, W., Chen, J., Olashaw, N., Zhang, X., Nicosia, S.V., Bhalla, K., and Bai, W. (2007b). SIRT1 sumoylation regulates its deacetylase activity and cellular response to genotoxic stress. Nature cell biology 9, 1253-1262.

Yaseen, N., and Blobel, G. (1999). GTP hydrolysis links initiation and termination of nuclear import on the nucleoporin nup358. Journal of Biological Chemistry 274, 2649326502.

Yasuda, R., Noji, H., Yoshida, M., Kinosita, K., and Itoh, H. (2001). Resolution of distinct rotational substeps by submillisecond kinetic analysis of F1-ATPase. Nature 410, 898904.

Yasuda, Y., Miyamoto, Y., Saiwaki, T., and Yoneda, Y. (2006). Mechanism of the stress-induced collapse of the Ran distribution. Experimental cell research 312, 512520.

Yoon, S.-O., Shin, S., Liu, Y., Ballif, B.A., Woo, M.S., Gygi, S.P., and Blenis, J. (2008). Ran-binding protein 3 phosphorylation links the Ras and PI3-kinase pathways to nucleocytoplasmic transport. Molecular cell 29, 362-375. 
Zhang, H., Saitoh, H., and Matunis, M. (2002). Enzymes of the SUMO modification pathway localize to filaments of the nuclear pore complex. Molecular and Cellular Biology 22, 6498-6508.

Zhang, J., Lian, Q., Zhu, G., Zhou, F., Sui, L., Tan, C., Mutalif, R.A., Navasankari, R., Zhang, Y., and Tse, H.-F. A human iPSC model of Hutchinson Gilford Progeria reveals vascular smooth muscle and mesenchymal stem cell defects. Cell Stem Cell 8, 31-45.

Zhou, B.-B.S., and Elledge, S.J. (2000). The DNA damage response: putting checkpoints in perspective. NATURE-LONDON-, 433-439.

Zhu, J., Zhu, S., Guzzo, C.M., Ellis, N.A., Sung, K.S., Choi, C.Y., and Matunis, M.J. (2008). Small ubiquitin-related modifier (SUMO) binding determines substrate recognition and paralog-selective SUMO modification. Journal of Biological Chemistry 283, 29405-29415.

Zorov, D.B., Filburn, C.R., Klotz, L.-O., Zweier, J.L., and Sollott, S.J. (2000). Reactive Oxygen Species (Ros-Induced) Ros Release A New Phenomenon Accompanying Induction of the Mitochondrial Permeability Transition in Cardiac Myocytes. The Journal of experimental medicine 192, 1001-1014.

Zorov, D.B., Juhaszova, M., and Sollott, S.J. (2006). Mitochondrial ROS-induced ROS release: an update and review. Biochimica et Biophysica Acta (BBA)-Bioenergetics $1757,509-517$. 